Issued by Sandia National Laboratories, operated for the United States Department of Energy by Sandia Corporation.

NOTICE: This report was prepared as an account of work sponsored by an agency of the United States Government. Neither the United States Government, nor any agency thereof, nor any of their employees, nor any of their contractors, subcontractors, or their employees, make any warranty, express or implied, or assume any legal liability or responsibility for the accuracy, completeness, or usefulness of any information, apparatus, product, or process disclosed, or represent that its use would not infringe privately owned rights. Reference herein to any specific commercial product, process, or service by trade name, trademark, manufacturer, or otherwise, does not necessarily constitute or imply its endorsement, recommendation, or favoring by the United States Government, any agency thereof, or any of their contractors or subcontractors. The views and opinions expressed herein do not necessarily state or reflect those of the United States Government, any agency thereof, or any of their contractors.

Printed in the United States of America. This report has been reproduced directly from the best available copy.

Available to DOE and DOE contractors from

Office of Scientific and Technical Information

P.O. Box 62

Oak Ridge, TN 37831

Prices available from (703) 605-6000

Web site: http://www.ntis.gov/ordering.htm

Available to the public from

National Technical Information Service

U.S. Department of Commerce

5285 Port Royal Rd

Springfield, VA 22161

NTIS price codes

Printed copy: A05

Microfiche copy: A01

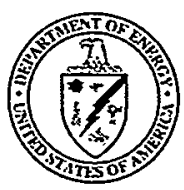




\section{DISCLAIMER}

Portions of this document may be illegible in electronic image products. Images are produced from the best available original document. 
SAND99-2512

Unlimited Release

Printed October 1999

\title{
A Statistical Description of the Types and Severities of Accidents Involving Tractor Semi-Trailers, Updated Results for 1992-1996
}

\author{
David B. Clauss \\ High Consequence Assessment and Technology Department \\ Sandia National Laboratories \\ P.O. Box 5800 \\ Albuquerque, New Mexico 87185-0767 \\ Daniel F. Blower \\ Center for National Truck Statistics \\ University of Michigan Transportation Research Institute \\ Ann Arbor, Michigan 48109-2150
}

\begin{abstract}
- This report provides a statistical description of the types and severities of tractor semi-trailer accidents involving at least one fatality. The data were developed for use in risk assessments of hazardous materials transportation. A previous study (SAND93-2580) reviewed the availability of accident data, identified the TIFA (Trucks Involved in Fatal Accidents) as the best source of accident data for accidents involving heavy trucks, and provided statistics on accident data collected between 1980 and 1990. The current study is an extension of the previous work and describes data collected for heavy truck accidents occurring between 1992 and 1996. The TIFA database created at the University of Michigan Transportation Research Institute was extensively utilized. Supplementary data on collision and fire severity, which was not available in the TIFA database, were obtained by reviewing police reports and interviewing responders and witnesses for selected TIFA accidents. The results are described in terms of frequencies of different accident types and cumulative distribution functions for the peak contact velocity, rollover skid distance, effective fire temperature, fire size, fire separation, and fire duration.
\end{abstract}




\section{Acknowledgment}

Barry Boughton and Toni Molina of Sandia National Laboratories, and Bob Pichler of the University of Michigan Transportation Research Institute made significant contributions to the supplemental data collection effort. In addition John Clauss and Bill Hartman of Sandia reviewed the document and provided helpful comments and suggestions. 


\section{Contents}

1. EXECUTIVE SUMMARY ..................................................................................11

2. INTRODUCTION..............................................................................................13

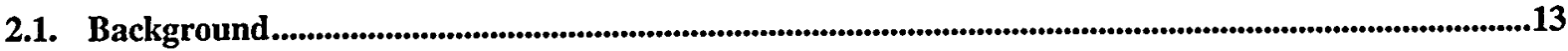

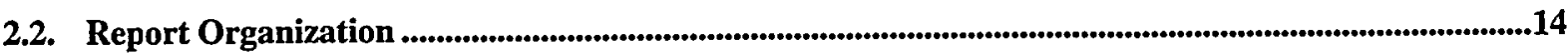

3. ACCIDENT DATA NEEDED IN RISK ASSESSMENTS .................................15

4. AT/SST AND AT/SGT SURROGATE DEFINITION ...........................................17

5. REVIEW OF EXISTING HEAVY TRUCK ACCIDENT FILES ...........................19

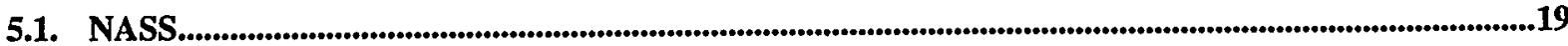

5.2. OMC

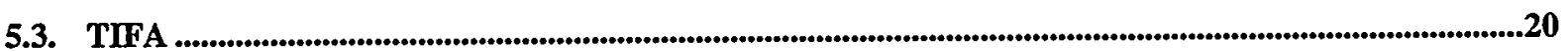

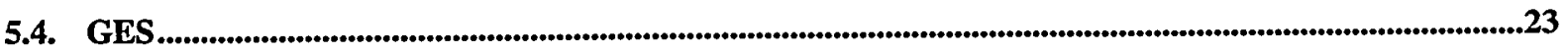

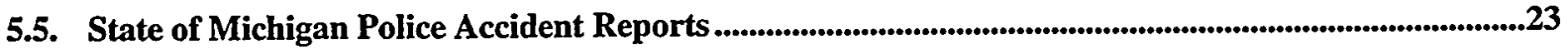

6. SUPPLEMENTAL DATA ...................................................................................25

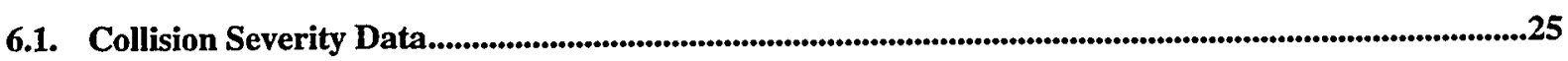

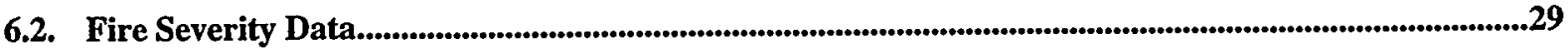

7. COMPILATION OF ACCIDENT FILES..............................................................33

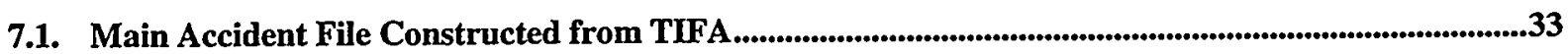

7.2. Accident File Constructed from GES Data ...........................................................................................36

7.3. Fire Severity Data - TIFA

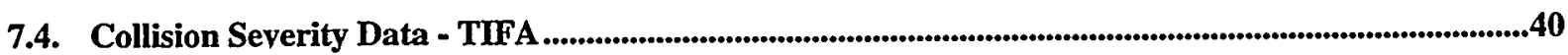

8. DISCUSSION AND ANALYSIS OF ACCIDENT TYPES ..................................43 


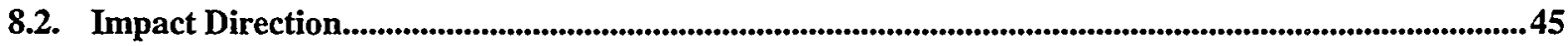

8.3. Other Vehicle Involved ...............................................................................................................................................48

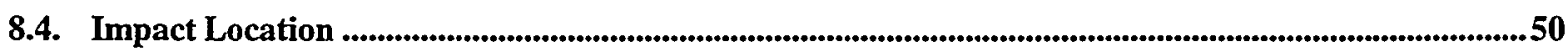

8.5. Rollover Occurrence ............................................................................................................................................53

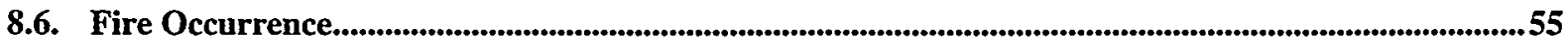

9. DISCUSSION AND ANALYSIS OF ACCIDENT SEVERITIES .........................57

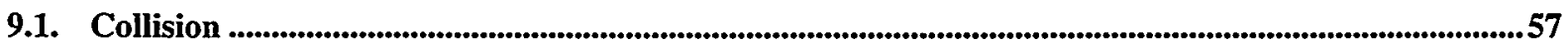

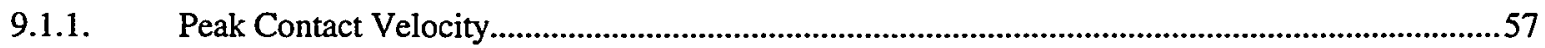

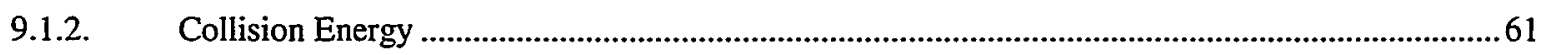

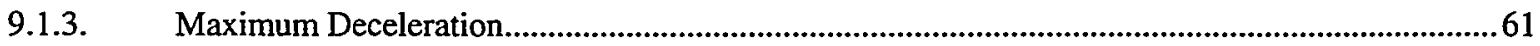

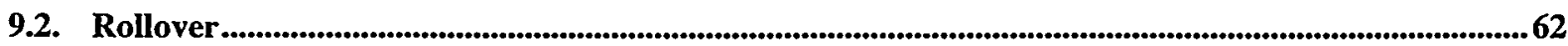

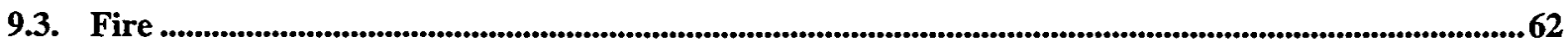

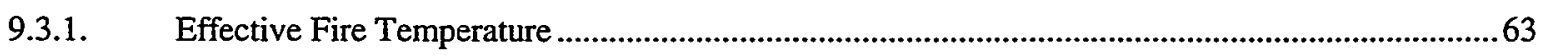

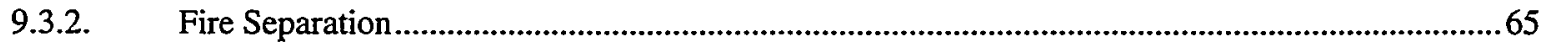

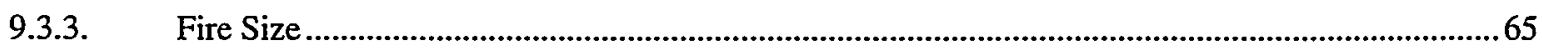

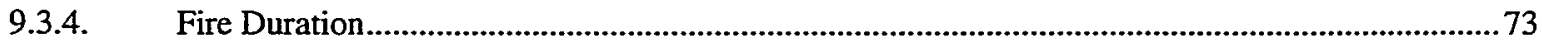

10. APPLICATION OF ACCIDENT DATA IN RISK ASSESSMENT ........................79

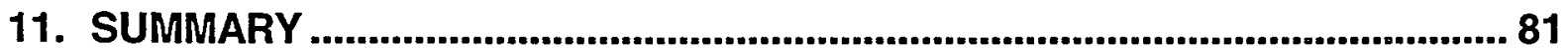

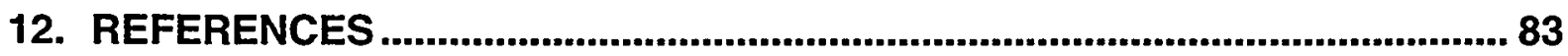

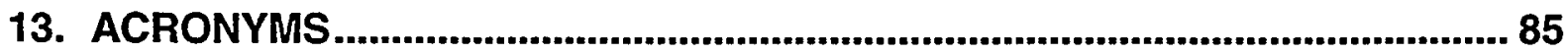

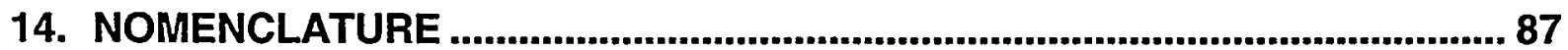




\section{Figures}

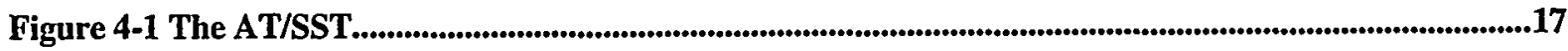

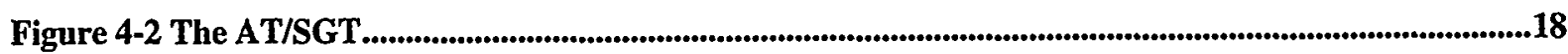

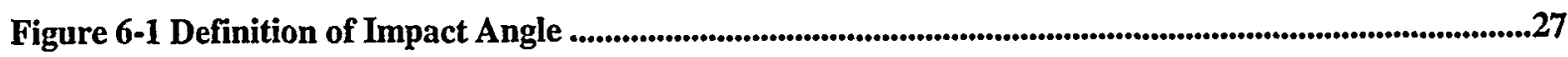

Figure 6-2 Codes for Contact Area on Tractor and Trailer ...........................................................................................29

Figure 6-3 Supplemental Data Form for Fire Severity (1992-1996) ......................................................................31

Figure 9-1 AT/SST Peak Contact Velocity Distributions by MHE, D=Front ..................................................59

Figure 9-2 AT/SST Peak Contact Velocity Distributions by MHE, $\mathrm{D}=$ Side.......................................................60

Figure 9-3 AT/SST Peak Contact Velocity Distributions by MHE, ID=Rear ....................................................60

Figure 9-4 Distribution for Skid Distance Associated with Rollover....................................................................62

Figure 9-5 Distribution for Effective Fire Temperature-.-Object Heated is in the Fire .......................................664

Figure 9-6 Effective Fire Temperature vs. Fire Diameter--Object Heated is out of the Fire..........................64

Figure 9-7 Distributions for Separation Distance by MHE....................................................................................66

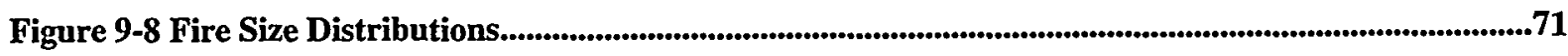

Figure 9-9 Comparison of Distributions for Fire Surface Area-.Simulation vs. Accident Data .......................73

Figure 9-10 Fire Duration Distributions (Other Vehicle Involved is Truck) ......................................................74

Figure 9-11 Fire Duration Distributions (Other Vehicle Involved is Car) ..........................................................75

Figure 9-12 Fire Duration Distributions (Other Vehicle Involved is Tanker) .....................................................75

Figure 9-13 Fire Duration Distributions (No Other Vehicle Involved) ....................................................................76

Figure 9-14 Fire Duration Distributions (Other Vehicle Involved is Train).........................................................76

Figure 9-15 Comparison of Distributions for Fire Duration--Simulation vs. Accident Data ............................77 


\section{Tables}

Table 6-1 Spreadsheet for Supplemental Collision Severity Data .......................................................................26

Table 6-2 Cell Formulas used in Spreadsheet for Collision Severity Data .........................................................26

Table 6-3 Codes for Road Surface and Corresponding Range for Coefficient of Friction ................................27

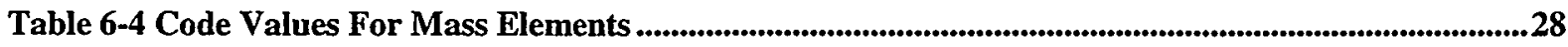

Table 6-5 Estimated Weights of Selected Fixed Objects .................................................................................29

Table 7-1 List of Variables in Main Accident File ............................................................................................34

Table 7-2 List of Variables in Accident File Constructed from GES .....................................................................37

Table 7-3 List of Variables in the TIFA Fire Severity File .................................................................................39

Table 7-4 States Selected for Review of Collision Severity Data .....................................................................41

Table 7-5 List of Variables in the TIFA Collision Severity Data File .....................................................................42

Table 8-1 Definition of MHE ....................................................................................................................................43

Table 8-2 Weighted Number of Fatal Accidents by MHE and Operating Environment .................................45

Table 8-3 Frequency of MHE by Operating Environment (Fatal Accidents) ...........................................................45

Table 8-4 Overall Frequency of each MHE for Different Accident Thresholds ..............................................46

Table 8-5 Weighted Number of Fatal Accidents by Impact Direction and MHE ...................................................47

Table 8-6 Frequency of Impact Direction by MHE (Fatal Accidents) ..............................................................44

Table 8-7 Overall Frequency of Impact Direction by MHE for Different Accident Thresholds....................49

Table 8-8 Weighted Number of Fatal Accidents in Which a Collision Occurred by MHE and

Other Vehicle Involved...........................................................................................................................50

Table 8-9 Frequency of Other Vehicle Involved by MHE (Given a Collision)......................................................50

Table 8-10 Weighted Number of Fatal Accidents by Impact Location, Direction and MHE ........................51

Table 8-11 Frequency of Impact Location by Impact Direction and MHE (Fatal Accidents).........................52

Table 8-12 Weighted Number of Fatal Accidents with Rollover by MHE and ID .........................................53 
Table 8-13 Frequency of Rollover by MHE and Impact Direction (Fatal Accidents) . .54

Table 8-14 Overall Frequency of Rollover by MHE and Accident Reporting Threshold................................54

Table 8-15 Weighted Number of Fatal Accidents with Fire by Impact Direction and MHE...........................55

Table 8-16 Frequency of Fire by MHE and Impact Direction (Fatal Accidents) ................................................56

Table 8-17 Overall Frequency of Fire by MHE for Different Accident Reporting Thresholds......................56

Table 9-1 Computation of Effective Mass for the AT/SST.................................................................................58

Table 9-2 Frequency of Separation Category by MHE (Fatal Accidents) .........................................................65

Table 9-3 Weighting Factors Used to Construct Fire Size Distributions ................................................................72

Table 9-4 Fatal Involvements by Other Vehicle Involved and MHE Given a Fire Occurrence .....................72 


\section{Preface}

This report describes the accident data for tractor semi-trailers developed for use in the Defense Programs Transportation Risk Assessment (DPTRA) project sponsored by the U. S. Department of Energy ${ }^{1}$. An earlier version of this report was published in June 1994 as SAND93-5280 in support of the original DPTRA report, which was approved in September 1994. Because the risk assessment must be updated on a five year cycle, the accident data report has been updated to provide current statistics on the types and severities of tractor semitrailer accidents. The organization and content of this report is similar (and in places, identical) to the previous report, with the primary differences being (1) the method of collecting supplemental TIFA data (see Chapter 6) and (2) the data years considered (see Chapters 7-10).

${ }^{1}$ The objective of the DPTRA project is to quantify the risk from inadvertent dispersal of radioactive material associated with off-site transportation of Defense Programs cargoes. 


\section{Executive Summary}

Quantitative risk assessments for hazardous materials transportation require analysis of the response of the transport vehicle, the package and its contents to the abnormal environments associated with a severe traffic accident to determine the conditions under which the hazardous material may be released into the environment. Statistical descriptions of the types of accidents that occur and of the severity of each type of accident are needed to evaluate the probability of releasing hazardous material to the environment.

This report provides such a description of the types and severities of accidents involving tractor semi-trailers. The primary data are based on traffic accidents involving at least one fatality. The information presented in this report was specifically developed to support a probabilistic assessment of the risk of transporting certain radioactive materials in the Armored Tractor (AT)/Safe-Secure Trailer (SST) for the U. S. Department of Energy (DOE). ${ }^{2}$ However, because the information is based on tractor semi-trailers used in general commerce, much of the information described in this report may be of interest to anyone evaluating the safety of transporting hazardous materials in tractor semi-trailers.

A number of previous studies have addressed transportation accidents involving heavy trucks with the same basic intent, purpose and approach as this report. The information on the types and severities of accidents in the current study is more detailed and more recent than that presented in previous studies, and it is also stored electronically, which simplifies queries and retrieval of the information.

A large number of accident variables can be used to characterize an accident. In this study, the emphasis was on defining the minimum number of accident characteristics that would provide a sufficient definition of the accident environment to analyze the response of the hazardous materials packaging system and also provide reasonable differentiation of the collision, rollover and fire severities. The variables used to characterize accidents were selected based in part on specific features of the packaging system used by the DOE to transport Defense Programs cargoes.

Distributions of accident types are defined by the types of vehicles or other objects involved, collision occurrence, angle of impact, location of principal impact, rollover occurrence and fire occurrence (Chapter 8). Distributions of peak contact velocity, skid distance, effective fire temperature, fire size, fire separation, and fire duration were developed to define the severity of the accident (Chapter 9). The report also includes a discussion of the application of this data in risk assessments for transportation of hazardous cargoes (Chapter 10).

\footnotetext{
${ }^{2}$ The project is known as the Defense Programs Transportation Risk Assessment (DPTRA). The DOE has also begun transporting hazardous materials in a newly-designed trailer, referred to as the SGT (Safeguards Transporter).
} 
--This page left almost blank intentionally-- 


\section{Introduction}

\subsection{Background}

Quantitative risk assessments for hazardous materials transportation require analysis of the response of the transport vehicle, the package and its contents to the abnormal environments associated with a severe traffic accident to determine the conditions under which the hazardous material may be released into the environment. Statistical descriptions of the types of accidents that occur and of the severity of each type of accident are needed to evaluate the probability of releasing hazardous material to the environment.

This report provides such a description of the types and severities of accidents involving tractor semi-trailers. This report covers data for accidents occurring between 1992 and 1996, and is an update of a report on data for accidents occurring between 1980 and 1990 [1]. The primary data are based on traffic accidents involving at least one fatality. Accident rates are described in a separate report $[2,3]$. The information presented in this report and in Reference 3 was specifically developed to support a probabilistic assessment of the risk of transporting certain radioactive materials in the AT/SST (or the AT/SGT) for the DOE. The operating history with the AT/SST includes only limited accident experience ${ }^{3}$. Although sufficient to describe an overall accident rate, the number of accidents in which AT/SSTs have been involved is insufficient to provide a meaningful statistical description of the types and severities of traffic accidents involving AT/SSTs. Therefore, it is assumed that, given an accident, the distributions of the types and severities of accidents involving tractor semitrailers that are used in general commerce and that have characteristics similar to those of an AT/SST or AT/SGT are representative of the distributions of the types and severities of accidents involving AT/SSTs and AT/SGTs. Consequently, much of the information described in this report may be of interest to anyone evaluating the safety of transporting hazardous materials in tractor semi-trailers.

There have been a number of previous studies that addressed transportation accidents involving heavy trucks and that had the same basic intent, purpose and approach as this report (e.g., References 4-6). The information in the current study has the following advantages:

- A more detailed characterization of accident types is provided.

- Collision and fire severity data are more detailed and more comprehensive.

\footnotetext{
${ }^{3}$ Since the SGTs have just recently been introduced, there is no accident experience at this time with the AT/SGT.
} 
- The data collection process was improved by reviewing the data more contemparaneously with the accident and by conducting interviews with accident witnesses and responders as necessary.

- The data are more recent and may therefore be more representative of the current environment for truck transportation.

- The data are stored in a set of electronic databases that can be easily queried and analyzed to obtain information in formats other than those used in this study.

\subsection{Report Organization}

The executive summary appears in Chapter 1. The background, purpose, and objectives for this report are discussed in Chapter 2. Chapter 3 provides a discussion of the type of data needed to evaluate the response of the transport vehicle, package and package contents to severe transportation accidents. Chapter 4 provides a brief description of the AT/SST and AT/SGT and identifies the features that a truck-trailer combination should have to serve as a useful surrogate. Chapter 5 provides a brief review of available accident databases. The procedures used to generate supplemental accident data are described in Chapter 6. The compilation of the accident data files used in this report, including the use of existing data files and the generation of supplemental data, are described in Chapter 7. The statistical descriptions of accident types and severities used in the DPTRA study appear in Chapters 8

and 9, respectively. The suitability of the accident data developed in this study for use in transportation risk assessments is discussed in Chapter 10. Chapter 11 provides an overall summary. References are listed in Chapter 12. Acronyms and nomenclature are listed in Chapters 13 and 14, respectively. 


\section{Accident Data Needed in Risk Assessments}

One element of a risk assessment for transportation of hazardous materials involves evaluation of the probability that the hazardous material will be released into the environment. Hazardous materials are typically shipped in a packaging system designed to mitigate accident environments and to prevent releases to the environment. In general, normal transportation environments do not produce environments that threaten the integrity of the packaging system. However, the environments produced from very severe traffic accidents could exceed the capabilities of the packaging system and cause a release of hazardous material.

Transportation risk assessments typically consider impact, puncture, crush and thermal environments. ${ }^{4}$ In traffic accidents, impact, puncture and crush environments are associated with collision and rollover events; thermal environments are associated with fires involving the fuel system, cargo or other elements of the vehicles and/or objects involved in the accident. The response of the packaging system to these environments is likely to be interdependent. For example, the response of the packaging system to a fire should reflect damage to the packaging system caused by collision and rollover.

The accident data needed to define the probability of packaging system failure include probabilities of various accident types and distributions of collision, rollover, and fire severity. The response of the packaging system and the collision, rollover and fire severity depend on the type of accident. There are a large number of accident variables that can be used to characterize the accident. In this study, the emphasis was on defining the minimum number of accident characteristics that would provide a sufficient definition of the accident environment to analyze the response of the packaging system and also provide reasonable differentiation of the collision, rollover and fire severities. As a result, the variables used to characterize accidents were selected based in part on specific features of the packaging system used by the DOE to transport Defense Programs cargoes.

The packaging system used by the DOE to transport Defense Program materials includes the cargo, containers and the SST or SGT. The most important features of the SST and SGT that protect the cargo in accident environments include the walls of the trailer (including the ceiling, floor and doors), which provide significant thermal protection in fire environments, and the cargo tie-down system, which provides a means of holding the cargo in place. In general, damage to the walls of a trailer in an accident depends on a number of factors. If the accident involves a collision, accident characteristics that may affect the response of the packaging system include the location of the principal impact, the impact direction, the collision energy absorbed, the peak contact velocity and the collision duration, which depends

\footnotetext{
${ }^{4}$ For some cargoes, immersion environments may also be a concern. Immersion is not evaluated in detail in this report. However, the raw data on fatal accidents in which the most harmful event is coded as immersion is presented in Reference 1.
} 
on the type of vehicles and/or objects involved. ${ }^{5}$ If the accident involves a rollover, the primary characteristic of the rollover that may affect response of the packaging system (primarily by damage to trailer walls) is the skid distance. If the accident involves a fire, additional accident characteristics that will affect the response of the packaging system include the effective fire temperature, the size of the fire, the separation between the fire and the trailer, the number of walls on the trailer that are directly heated by the fire, and the duration of the fire.

The types of vehicles and/or other objects involved, collision occurrence, angle of impact, location of principal impact, rollover occurrence and fire occurrence are factors that define the types of accidents. These factors are discussed in more detail in Chapter 8. The peak contact velocity, collision energy absorbed, skid distance, effective fire temperature, fire size, fire separation, the number of heated walls and fire duration are factors that define the severity of the accident. These factors are discussed in more detail in Chapter 9.

The accident characteristics identified in the preceding discussion represent a compromise between the accident data that was available or could be generated (see Chapters 5 and 6) and the rigor of the analysis used to analyze the response of the packaging system. Detailed engineering data are almost never available for vehicles involved in traffic accidents and so relatively simple, approximate methods of analysis are needed.

${ }^{5}$ Specifically, damage to the trailer walls depends on the location of the principal impact, the impact direction and the collision energy absorbed, and damage to the container and cargo depends on the peak contact velocity and the collision duration. 


\section{AT/SST and AT/SGT Surrogate Definition}

As described in Section 2.1, the lack of sufficient accident data with the AT/SST and AT/SGT necessitates the use of a surrogate for which sufficient data are available to develop a statistical description of the types and severities of accidents. The ideal surrogate for the AT/SST or AT/SGT would be a tractor semi-trailer that has similar features, is operated in a like manner, and is widely used. The selection of the actual surrogate represents a compromise between the definition of an ideal surrogate, the nature of the available data, and the need for a large sample size.

Figure 4-1 is a photograph of an AT/SST and Figure 4-2 is a photograph of an AT/SGT. Both vehicles are five-axle, tractor semi-trailer combinations, consisting of a three-axle power unit pulling a two-axle, 40-foot van semi-trailer. The vehicles typically operate at a gross combination weight (GCW) of between approximately 65,000 and 80,000 pounds. ${ }^{6}$ The AT/SST and AT/SGT are both highly specialized vehicles with many features that set it apart from the general truck population, including its brake system, state-of-the-art suspension system, cargo tie-down system and automatic transmission.

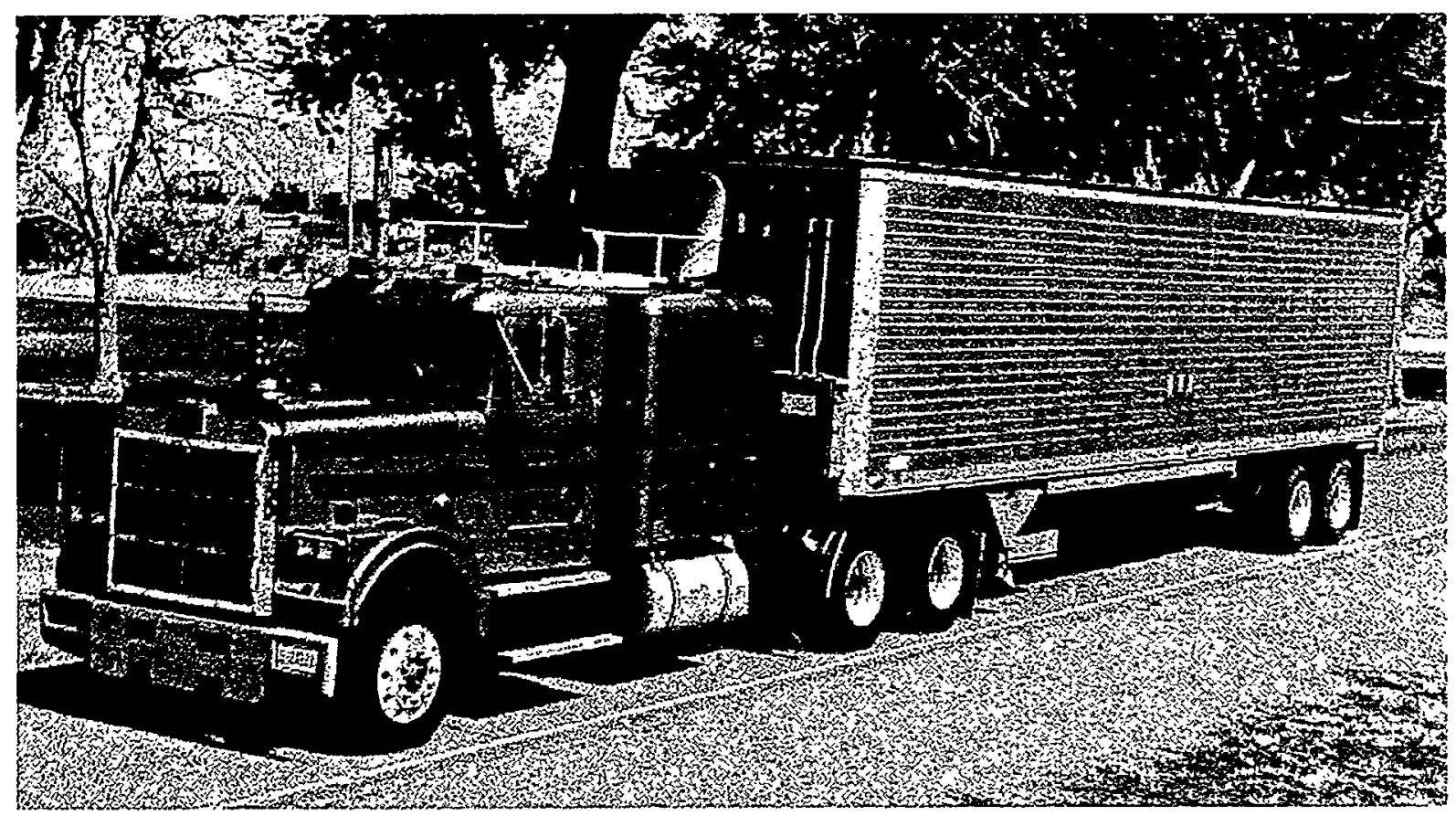

Figure 4-1 The AT/SST

${ }^{6}$ The base curb weight of the AT is 24,200 lbs. The base curb weight of an SST is between 40,500 and 41,500 Ibs, depending on the specific model. The base curb weight of an SGT is $41,300 \mathrm{lbs}$. Base curb weight does not include the weight of drivers, passengers, or cargo. 


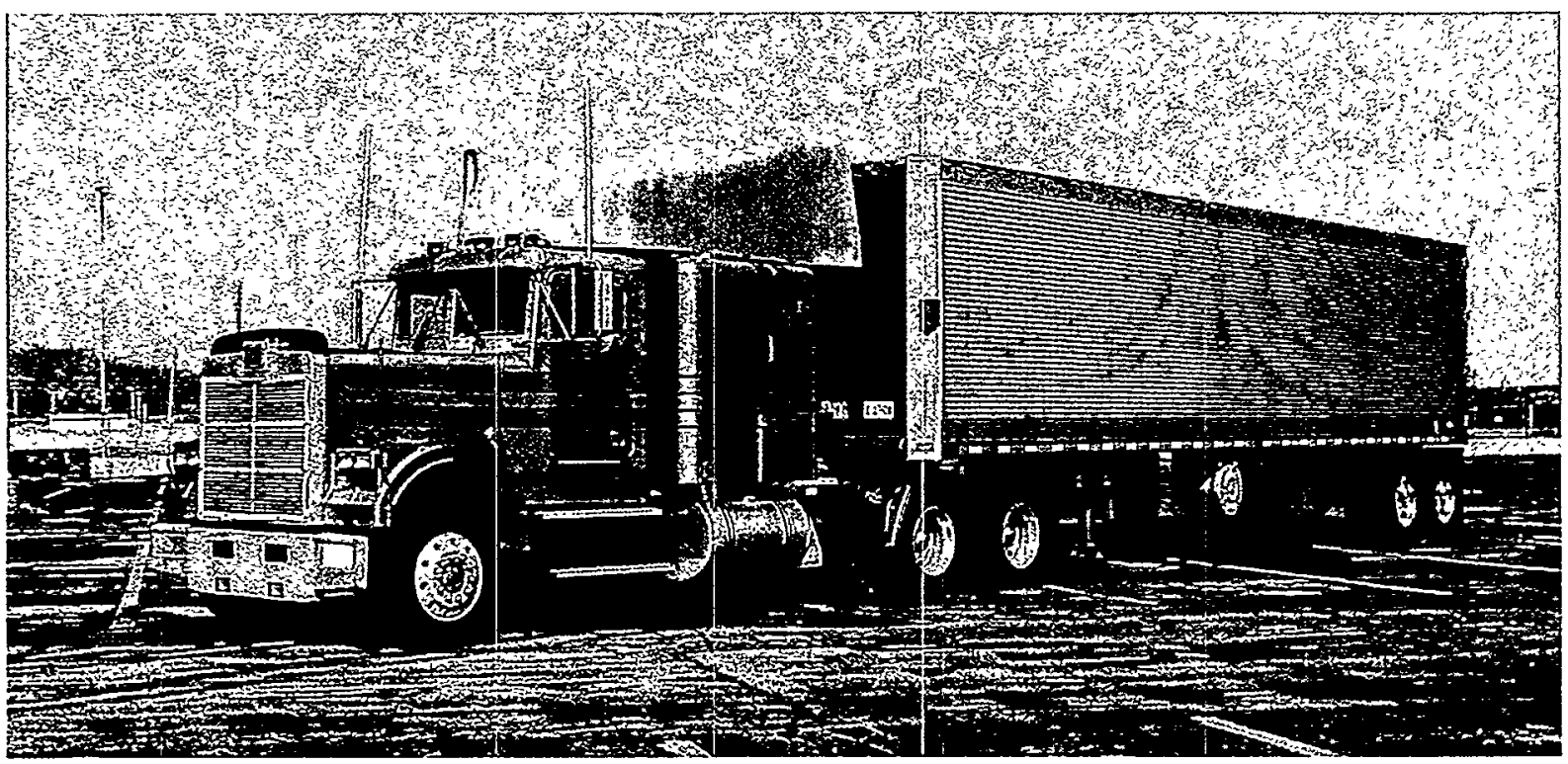

Figure 4-2 The AT/SGT

AT/SSTs and AT/SGTs are operated in convoys with escort vehicles, and are driven mainly over interstate and other highways. Routes are chosen to avoid population centers and operations are restricted to avoid bad weather. If weather conditions create an unsafe driving environment, convoys seek "safe haven" until weather and road conditions return to a safe level. Shipments are made in accordance with all federal regulations governing the transportation of hazardous materials. The couriers who operate AT/SSTs and AT/SGTs are highly-trained, federal officers who must pass regular health, physical-fitness, and driving examinations. Their driving shifts are limited to four hours, and no courier may be on the road continuously for more than 32 hours. That is, each courier is required to get eight hours of "bed rest" at intervals not to exceed 32 hours.

The physical features and maintenance of the AT/SST and AT/SGT, training of the couriers, and operating procedures have the effect of minimizing transportation risk; in particular by lowering the overall accident rate and, when there is an accident, by producing less-severe accidents. These factors are accounted for in the overall accident rate for the AT/SST and AT/SGT used in the risk assessment since the rate is based on the actual operating experience of the AT/SST. However, the distributions of the types and severities of accidents are taken to be independent of the special features of the AT/SST and AT/SGT. Similarly, we ignored operating procedures, maintenance, and driver training even though they are also factors in reducing accident severity. Consequently, the criterion used to define a surrogate for the AT/SST and AT/SGT was based on the most basic, outward physical features of the vehicles, i.e., any tractor semi-trailer combination involved in an accident was used as a surrogate for the AT/SST and AT/SGT. For the most part, the data files actually allow for a more precise definition of the surrogate (e.g., trailer body style, axle count and GCW could also be considered) but the sample sizes become too small when the definition of the surrogate is further restricted. 


\section{Review of Existing Heavy Truck Accident Files ${ }^{7}$}

There are only a few publicly available, nationally representative accident data files that deal with truck accidents:

1. The Office of Motor Carriers (OMC) MCS 50-T file from the Federal Highway Administration (FHWA);

2. The National Accident Sample Survey (NASS) file from the National Highway Traffic Safety Administration (NHTSA);

3. The Trucks Involved in Fatal Accidents (TIFA) file from the University of Michigan Transportation Research Institute (UMTRI); and

4. The General Estimates System (GES) file from the NHTSA.

The following sections provide a description of each of these files and an indication of their utility in providing surrogate accident data for an AT/SST.

\subsection{NASS}

The NASS file [7] is a probability based sample of police-reported accidents. Sample cases are drawn by means of a three-stage process: first, Primary Sampling Units (PSU) are sampled from approximately 1200 political jurisdictions; then, police jurisdictions are sampled from within each sampled PSU; finally, within the sampled police jurisdictions, police accident reports are sampled. The sampled cases are investigated in great depth by teams of accident reconstruction experts. Because of the great investigative depth, NASS includes relatively few cases. Moreover, the focus of the work has been primarily on passenger automobiles. Consequently, sample size for truck accidents is small. NASS includes about 500 cases per year between 1980 and 1986 that can be identified as a tractor semi-trailer. This is too few to support all data elements required in the risk assessment. In addition, since there are so few cases, sampling errors are large and the reliability of the estimates correspondingly small. Finally, in 1987 NASS dropped large trucks entirely in order to focus on passenger cars. Therefore, the NASS file was not used in the present study.

\subsection{OMC}

The OMC accident file [8] is compiled from reports submitted by involved parties. All interstate carriers, whether public or private, who operate medium or heavy trucks are required

\footnotetext{
${ }^{7}$ This chapter is almost identical to the corresponding chapter in Reference 1 and is included here primarily for continuity and completeness.
} 
to file an MCS 50-T report on traffic accidents involving a fatality, injury, or property damage over a given amount ( $\$ 4,400$ at the time this study was conducted). These reports are completed by the carrier itself, typically by the safety director. The OMC file includes sufficient detail about the truck to identify an AT/SST surrogate with some specificity. The file includes variables about trailer type, cargo-body style, axle count for each unit, and GCW. However, fire is coded only as part of a hierarchical variable, so some fires cannot be identified. Similarly, rollover can be identified only for single-vehicle accidents. Finally, OMC cannot support identification of the collision types required in the risk assessment.

The OMC file has other problems as well, related to representativeness. First, only interstate carriers are required to file reports. Public and private carriers who operate only within a given state are exempt from the reporting requirement. Second, enforcement of the reporting requirement is weak. The comprehensiveness of the TIFA file (discussed in Section 5.3) makes it a good yardstick for measuring other files. Using the TIFA file as a standard, it can be shown that only about 60 percent of reportable fatal accidents, where reportable means that an interstate carrier was involved, are actually filed with the OMC. Since reporting is so low for the most serious accidents, it is believed that an even lower proportion of non-fatal accidents are reported. ${ }^{8}$ Because reporting is so low and because OMC covers only interstate operations, OMC is not a representative source for non-fatal accidents. As described in Section 5.3, information in the OMC file for fatal accidents is used to construct the TIFA file. Therefore, the OMC file was not used directly in this study.

\subsection{TIFA}

The TIFA survey data [9] provided much of the accident data needed in the risk assessment. TIFA includes all variables necessary to identify AT/SST surrogate vehicles. The file includes a large number of variables that can be used to categorize the types of accidents that occur, including most harmful event, impact direction, rollover occurrence, and fire occurrence. The file also includes all variables necessary to establish the operating environment. ${ }^{9}$ Finally, the TIFA is nationally representative in that it includes records for virtually all medium and heavy duty trucks involved in a fatal accident in the U.S. from 1980 to 1996, amounting to 52,450 tractor semi-trailer involvements. The primary drawback of the TIFA file is that includes only accidents in which there was at least one death. Accidents involving only injury and/or property damage are excluded. Nevertheless, the TIFA file does include most of the accidents of concern. The goal of this study was to determine the probability of traffic accidents that

\footnotetext{
${ }^{8}$ Because of data and representation problems, the reporting requirement for OMC was dropped in March 1993.

${ }^{9}$ The operating environment is defined by the road type and population area. Accident rates and distribution of accident types depend on the operating environment. A more detailed discussion of the significance of operating environment and how it is defined can be found in Section 8.1 and References 2 and 3.
} 
pose a serious threat to the physical integrity of the AT/SST and its cargo. Such accidents generally involve high-speed collisions with massive vehicles or objects, or major fires. These types of accidents are also the most likely to involve a fatality, so the set of accidents of concern is largely, although not necessarily entirely contained in the set of accidents involving a fatality. Chapter 10 provides a more detailed discussion of the significance of this limitation and how it can be addressed in a risk assessment.

TIFA is produced by the Center for National Truck Statistics at UMTRI. In 1981, UMTRI initiated a survey of all large trucks involved in fatal accidents in the continental United States. The first data year was 1980. The survey combines information from the Fatal Accident Reporting System (FARS) of the U. S. Department of Transportation NHTSA [10] with data from the OMC MCS 50-T reports (until the 1993 data year, when the MCS 50-T program concluded), state police accident reports, and comprehensive follow-up telephone interviews conducted by UMTRI research staff. At the time the current study was conducted, the file was complete through 1996.

The TIFA file covers all cases where a medium or heavy truck was involved in a fatal accident. Medium and heavy duty trucks cover non-passenger vehicles with a gross vehicle weight rating (GVWR) greater than 10,000 pounds. In essence, the file covers all trucks larger than a pickup. Cases for TIFA are originally identified by subsetting the appropriate vehicles from the FARS file. Police reports are obtained for all accidents from the states. Next an attempt is made to match FARS cases with their corresponding cases in the OMC MCS 50-T file. OMC reports provide extensive information on the physical configuration of the case vehicle, so OMC-matches are considered complete, except for checks on the completeness and consistency of the data. However, the matching process is only successful for about onethird of the FARS cases. For the remaining two-thirds of the cases, a follow-up telephone survey is conducted to collect a detailed physical description of the involved truck. The owner, driver, or some other involved party is contacted for information about the configuration of the truck, the number of trailers, cargo-body type, and many other details.

Every case produced by the telephone survey is subjected to extensive editing to ensure accuracy and completeness of the data. The VIN (vehicle identification number) is decoded to positively identify the vehicle, and the physical description from the phone interview is compared with the manufacturer's specifications. Inconsistencies or contradictions are resolved by further interviews, whenever possible. Computerized consistency checks are made on the entire file. Where problems are found with OMC cases, calls are made to resolve them. The result is a file with low missing data rates and high expectations of accuracy.

For data years 1980 through 1986, TIFA is a census file, meaning that it contains records for all medium and heavy trucks involved in a fatal accident. Data years 1987 through 1996 include some limited sampling, except 1993 which was a census file. The purpose of the sampling was to reduce the number of cases requiring telephone interviews, while not compromising the quality and completeness of the data. The raw number of cases for the data years where sampling was done is about 1,000 records fewer than if all cases had been taken. 
Appropriate weights have been determined that allow national population totals to be estimated. The sampling procedure is described below. Statistical work has shown that sampling has had little effect on the accuracy of estimates from the files [16]. The 1980-1996 TIFA file contains records on 44,655 tractor semi-trailer combinations, with a weighted total of 52,450 tractor semi-trailers.

The sampling algorithm is straightforward. For data years where an MCS 50-T report was available, all OMC-matched cases are included in the file with certainty and have a weight of one. The pool of cases to be sampled covers straight trucks with no trailer or tractors with one trailer, which are identified in FARS with the greatest accuracy. The cases to be sampled are sorted by state and month to ensure good annual coverage, and an interval selection procedure is employed, taking every other case. A weight of two is given to the cases thus selected. All other cases (non-OMC-matches and not a straight truck with no trailer or tractor with one trailer) are taken with certainty and have a weight of one. Thus, the sampling procedure is limited to cases with no OMC-match and that are either a straight truck with no trailer or a tractor semi-trailer. Interviewing on the sampled cases then proceeds normally.

FARS includes a long list of variables about the accident environment, the events of the accident, and the people involved, but the detail about the vehicles involved is limited. The FARS accident, vehicle, and person variables relating to the truck are all incorporated in the TIFA file. TIFA also includes the detailed physical description of the involved truck from the MCS 50-T report, where a match could be found, or, if not, the even greater detail of the telephone survey. Consequently, the TFFA file contains the most complete selection of variables and the most detailed physical description of the vehicles involved available. Virtually all of the data elements identified related to types of accidents are in the TIFA file. Information about the "other vehicle involved" is not included in TIFA, but it was reconstructed from the original FARS file, since FARS includes all vehicles, not just trucks, involved in a fatal accident. A version of the TIFA file has been built that incorporates this additional information. For each truck record in the TIFA file, variables have been added that record the number of passenger cars/light trucks/pickups, medium or heavy trucks, hazardous tankers, or other vehicles involved in the accident.

The TIFA file was also used for the fire and collision severity studies. UMTRI reviewed police reports and conducted phone interviews with witnesses and/or responders for TIFA cases between 1992 and 1996. All fire cases were examined and samples of the collision cases were drawn and examined. This topic is described further in Chapters 6 and 7.

The primary drawback to using TIFA exclusively in the risk assessment is that the file is limited to fatal accidents only. There is no information about non-fatal accidents in TIFA. This is not an insuperable problem since fatal accidents are typically serious accidents that involve high collision energies and severe fires, i.e., those that are likely to pose the greatest threat to the AT/SST. Evaluating the full range of threats to the AT/SST, however, requires some consideration of severe, non-fatal accidents in addition to fatal accidents (see Chapter 10). 
For the current study, TIFA data for the period 1992-1996 was used. The 1992-1996 TIFA file contains records on 9,220 tractor semi-trailer combinations, with a weighted total of 13,522 tractor semi-trailers.

\subsection{GES}

The GES file [11] is another probability-based sample of police-reported accidents. The GES file was developed by NHTSA to overcome the sample-size limits of the NASS file and provide reliable national estimates of accident totals. While NASS provides detailed examinations of particular accidents, GES is coded entirely from police accident reports. The GES file allows some extension of the risk assessment to non-fatal accidents. GES includes a shorter list of variables but larger sample size, including large trucks. Sample sizes are not as large as would be desirable but they are adequate. Five years of GES data (1992-96, which contained records for 24,355 tractor semi-trailers representing 891,190 vehicles) were used in the current study.

GES uses a three-stage sampling protocol similar to that of NASS. The United States is divided into PSUs grouped by geographical region (Northeast, South, Central and West) and type (large central city, large suburban areas and other). Police jurisdictions are sampled within the geographical areas in the second stage. Finally, GES investigators periodically visit the sampled police jurisdictions and select cases for inclusion in the GES file. Accidents are filed into three groups (involving a towed vehicle, not involving a towed vehicle but including an injury, and other), and a random sample is drawn from each group. In 1990, changes were implemented to increase the sample of large trucks. The GES variables are then coded from the selected police reports. Unlike NASS, there is no further investigation of the cases.

The GES file does not contain all the vehicle-level detail of TIFA so the most precise surrogate definition that can be used is a tractor semi-trailer. There is no information in GES for cargo-body type, axle counts, or GCW. GES includes many of the same variables as the FARS. Make/model, most harmful event, rollover, fire, and time of day are identical in the GES, TIFA and FARS files. With the make/model coding, the type of other vehicles involved can be identified. Unfortunately, the road type and area type variables in GES are unusable. The Interstate variable codes cover only roads that are explicitly identified as a numbered Interstate highway, so many named Interstates are not identified and, of course, other limited access roads are not included. The smallest population size distinguished in the urban/rural variable is a city of 25,000 people, far larger than the FHWA rural definition of 5,000 people. Consequently, the dependence of accident data on road type and area type cannot be determined using GES.

\subsection{State of Michigan Police Accident Reports}

Police accident report files can be readily acquired from the state of Michigan by UMTRI and consist of all reportable traffic accidents. The data are collected by police officers; additional 
information is sometimes coded subsequently from the police report. At the time this study was conducted the reporting threshold in Michigan was $\$ 200$ of property damage.

The primary limitation of the state of Michigan file [12] is that it may not be representative of the national experience. In addition, the amount of detail on truck configuration is limited. Only tractor semi-trailers can be identified. There is no information about cargo-body style, axle count, or GCW. Rollover can be identified for single-vehicle accidents. Michigan has a usable fire variable, which includes information on the source of fire. "Object Struck" variables are reasonably complete; and, since the files are census files, there are a large number of cases available. In the original study [1], the Michigan file was used to gain insight into the severity of non-fatal accidents, as compared to fatal accidents. The Michigan file was used primarily as a source of hard copies of police reports that were studied for information on accident severities, for example, the duration of fires. With this focus, the question of national representativeness was less of an issue. 


\section{Supplemental Data}

The computerized databases that are available contain variables that can be used to determine the frequencies of most of the accident types that are needed to define the accident environment in transportation risk assessments (see Chapter 3). However, describing the severity of accidents, fire and collision severity in particular, requires information not contained in these databases. Therefore, procedures for gathering supplemental information on collision and fire severity were developed. Cases of interest were identified from the TIFA. Data were entered into a spreadsheet for collision severity and onto a data form for fire severity based on a review of the information available in the police reports, supplemented by phone interviews with accident witnesses and responders (as necessary) for the selected cases. The data obtained from the police reports were independently reviewed and checked for accuracy.

Section 6.1 describes the information entered into the spreadsheet, the procedures used to obtain this information and the spreadsheet calculations for the collision severity data. Section 6.2 describes the information entered onto the fire severity data form and the procedures used to obtain this information. Chapter 7 provides a description of the cases selected from the TIFA data file and the resulting database files.

The TIFA supplemental data described in the body of this report covers years 1992-1996. Because the supplemental data described in this report was collected in a more timely fashion than the supplemental data described in Reference 1, and because phone interviews were conducted, the number of cases for which variables such as fire size and fire duration are known or can be estimated is greater than described in Reference 1. Furthermore, since better information is generally available, a single estimate is used for each variable of interest as opposed to the previous effort, for which upper bound, lower bound, and average estimates were used to describe many variables. In addition, refinements have been made in the types and quality of information collected and the processes used to collect the information.

\subsection{Collision Severity Data}

Supplemental data for collision severity are collected based on information from police reports and phone interviews conducted by UMTRI. The data are entered into a spreadsheet, which is then used to compute measures of collision severity. Table 6-1 shows the format of the spreadsheet used to enter these data. Numbers in italicized text must be input; numbers in plain text are calculated. Rows 3 and 8 of the spreadsheet contain information on the case vehicle; rows 4 and 9 contain information on the other vehicle or object involved in the collision. For each case vehicle, only one event is coded.

The year (Yr), state code (St), case number (Case) and vehicle number (Veh\#) are input so that the supplemental data can be combined with data in the TIFA file. The travel speed (Trav Spd, mph), skid distance (Skid Dist., ft) and coefficient of friction (Coef. Fric.) are used to 
calculate the speed at impact (Impct Spd, $\mathrm{mph}$ ), using the spreadsheet formulas indicated in Table 6-2. The derivation of the formula used for impact speed is described in Reference 13. The impact angle (Angl, degrees), which is the angle subtended by the velocity vectors of the case vehicle and the other vehicle or object (see Figure 6-1), is used to calculate the relative velocity (Rel Veloc., $\mathrm{mph}$ ) between the case vehicle and the other vehicle or object. The weights of the case vehicle and the other vehicle or object are specified in lbs. and are used in the calculation of peak contact velocity $(\mathrm{PCV})^{10}$. The derivation of the formulas used for relative velocity and the peak contact velocity can be found in Reference 14.

Table 6-1 Spreadsheet for Supplemental Collision Severity Data

\begin{tabular}{|c|c|c|c|c|c|c|c|c|c|c|c|c|}
\hline & $\mathrm{A}$ & B & $\mathrm{C}$ & $D$ & $\mathrm{E}$ & $\mathrm{F}$ & $\mathrm{G}$ & $\mathrm{H}$ & I & $\mathrm{J}$ & $\mathrm{K}$ & $\mathrm{L}$ \\
\hline 1 & \multicolumn{4}{|c|}{ ESTIMATES } & Trav & Skid & Coef. & Impct & & Rel. & & \\
\hline 2 & $\mathrm{Yr}$ & St & Case & Veh\# & Spd & Dist. & Fric. & Spd & Angl & Veloc. & Weight & $\mathrm{PCV}$ \\
\hline 3 & 0 & 0 & 0 & 0 & -1 & 0 & 0 & -1.0 & 0 & 0.0 & 0 & -1.000 \\
\hline 4 & 0 & 0 & 0 & 0 & 0 & 0 & 0 & -1.0 & & & 0 & -1.000 \\
\hline 5 & & & & & & & & & & & & \\
\hline 6 & & & & & & & road & mass & area & area & object & \\
\hline 7 & & & & & & & surface & elements & tractor & trailer & struck & \\
\hline 8 & & & & & veh & 0 & 0 & 0 & 0 & 0 & $n / a$ & \\
\hline 9 & & & & & veh & 0 & & 0 & 0 & 0 & $n / a$ & \\
\hline 10 & & & & & & & & comments & none & & & \\
\hline
\end{tabular}

Table 6-2 Cell Formulas used in Spreadsheet for Collision Severity Data

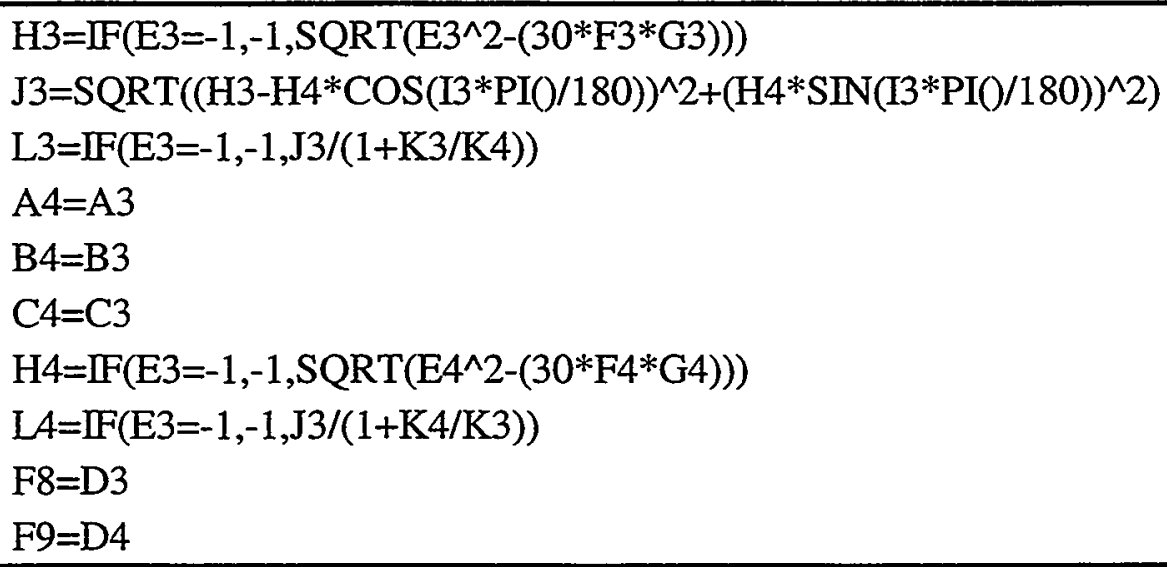

\footnotetext{
${ }^{10}$ The peak contact velocity is the maximum change in velocity that would be experienced by an unrestrained object in the vehicle; it is derived from conservation of momentum.
} 


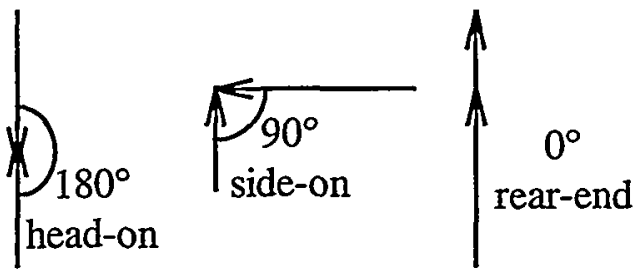

Figure 6-1 Definition of Impact Angle

Accidents in which there was no single collision that caused a sudden, large change in velocity are considered sideswipes. Sideswipes are characterized by glancing impacts and a decrease in velocity over an extended time and distance. These types of collisions do not constitute a threat to the integrity of the trailer or the cargo restraint systems. Travel speed is coded as -1 for the case vehicle if the accident is considered to be a sideswipe, which effectively classifies the collision event as non-threatening. For accidents classified as sideswipes, the skid distances, coefficient of friction, impact angle and weights are not used.

If the case vehicle was involved in more than one collision, the collision producing the greatest change in velocity is coded. If there is no collision event, the travel speed is coded as -2 . If there is a non-sideswipe collision event and the travel speed is unknown, it is coded as -9. Skid distance is entered only if explicitly known (skid distance may corresponding to tire marks or sliding of the vehicle on its side). If impact speed is explicitly specified in the police report, the skid distance and/or travel speed are adjusted such that the formula for impact speed gives the value indicated in the police report. In the case of impacts with fixed objects such as a wall or cliff, impact speed is the component of the truck's velocity that is perpendicular with the cliff. For these cases, travel speed is input to the spread sheet as travel speed times the sine of the angle between the vehicles direction of travel and a vector perpendicular to the surface of the object.

The coefficient of friction is based on the road surface, as indicated in Table 6-3. The code values for road surface are also indicated in this table.

Table 6-3 Codes for Road Surface and Corresponding Range for Coefficient of Friction

\begin{tabular}{|c|c|c|}
\hline Road surface & Code value & Coefficient of Friction \\
\hline Dry & 1 & $0.4-0.5$ \\
\hline Loose gravel & 2 & $0.3-0.4$ \\
\hline Wet & 3 & $0.3-0.4$ \\
\hline Snowy/Icy & 4 & $0.2-0.3$ \\
\hline Unknown & 9 & \\
\hline
\end{tabular}

The weight of the vehicle or object used in the spreadsheet is the effective weight from the standpoint of momentum transfer. The process for estimating effective mass has been simplified from the approach used in Reference 1. For vehicles, estimates are incremented only by the weight of an entire unit of the combination. That is, in the case of a tractor-semi- 
trailer, the effective mass is either the weight of the tractor, the trailer, or their sum, all of which are available in the TIFA file. In the case of a straight truck, the effective mass is the weight of the vehicle. The code values used to represent these mass elements are listed in Table 6-4. For fixed and non-fixed objects, the mass element is coded as 4 for not applicable. Also, if travel speed is "-2" (no collision), the mass elements are coded as 4.

Table 6-4 Code Values For Mass Elements

\begin{tabular}{|c|c|}
\hline Mass Element & Code Value \\
\hline Entire vehicle & 1 \\
\hline Tractor only & 2 \\
\hline Trailer only & 3 \\
\hline Not applicable & 4 \\
\hline Unknown & 9 \\
\hline
\end{tabular}

The following guidelines are used to evaluate the effective weight of vehicles other than medium or heavy trucks, objects, and trains:

1. The weight of large cars and pickups is assumed to be between 3500 and $4500 \mathrm{lb}$. depending on the model.

2. The weight of mid-size cars is assumed to be between 3000 and $4000 \mathrm{lb}$. depending on the model.

3. The weight of small cars is assumed to be between 2000 and $3500 \mathrm{lb}$. depending on the model.

4. The weight of motorcycles is assumed to be less than $1500 \mathrm{lb}$.

5. For fixed objects, the weight is set to an arbitrarily large number $\left(1 \times 10^{12} \mathrm{lb}\right.$.) if the object remained in place to reflect the fact that the velocity of the fixed object does not change in the collision. If the object did not remain in place (e.g., a telephone pole could be snapped off at its base), the weight is estimated at ten times the estimated actual weight of the object. Typical estimates for the weights of fixed objects that do not necessarily remain fixed are given in Table 6-5.

6. For non-fixed objects, the effective weight is generally taken to be less than $1500 \mathrm{lb}$.

7. For trains, the effective weight is based on the number of locomotives and cars participating in the momentum exchange. In collisions in which the case vehicle was struck by the train; the effective weight is assumed to equal the total weight of all locomotives and cars in the train. In collisions in which the case vehicle struck the train, the effective weight is set equal to the combined weight of the locomotive or car struck and the locomotive(s) or car(s) immediately in front of and in back of the one struck (if present). Locomotives are assumed to weigh $350,000-400,000 \mathrm{lb}$., empty box cars 60,000 lb., loaded box cars 180,000-200,000 lb., and passenger cars 100,000 lb. If a car is not identified, it is assumed to be a boxcar. 
The cells labeled "area tractor" and "area trailer" are used to define the contact area for the major impact. Figure 6-2 indicates the codes to be used for the tractor and trailer. For example, an opposite-direction sideswipe might be coded 1 . A rear-end might be 7 . An impact on the saddle-tanks of the tractor, just behind the cab, might be coded 6. For tractors or trailers that are not directly impacted and for objects, code 98 is used. If the travel speed is coded "-2" (non-collision), code 98 is entered. If the impact location is unknown, code 99 is used.

Table 6-5 Estimated Weights of Selected Fixed Objects

\begin{tabular}{|c|c|c|}
\hline Object & Actual Weight, lbs. & Effective Weight, lbs \\
\hline Tree & $500-2000$ per tree & $5000-20,000$ per tree \\
\hline Guardrail & $500-1000$ & $5000-10,000$ \\
\hline Telephone pole & 1000 & 10,000 \\
\hline Light pole & 1500 & 15,000 \\
\hline
\end{tabular}

Tractor

\begin{tabular}{|l|l|l|}
\hline 3 & 5 & 8 \\
\hline 2 & & 7 \\
\hline 1 & 4 & 6 \\
\hline \multicolumn{3}{|c|}{9 (top) } \\
10 (bot)
\end{tabular}

Trailer

\begin{tabular}{|l|c|l|}
\hline 3 & 5 & 8 \\
\hline 2 & 9 (top) & 7 \\
\hline 1 & 10 (bot) & 6 \\
\hline
\end{tabular}

Figure 6-2 Codes for Contact Area on Tractor and Trailer

The cells labeled "object struck" are used to enter an alpha description of the object struck. The description is limited to 255 characters. One of the purposes of this field is to enable us to revisit cases as we learn more and are able to make better decisions about the effective masses of fixed objects. By entering a fairly complete description, it will be possible to search for cases and ensure consistency. The description includes details that pertain to the effective mass. An additional cell is also available for general comments on collision severity.

\subsection{Fire Severity Data}

Figure 6-3 shows the new supplemental data form used for collecting fire severity data. This information is extracted directly from police accident reports and phone interviews, independently reviewed and checked for accuracy, and then typed into the existing data file. The data fields include the year, state number, case number and vehicle number so that the data can be combined later with data in the TIFA file.

Each fire in the accident is assigned a unique number (Fire No.). The number of fires coded for a specific case vehicle takes into consideration how the nature of the fires might have 
differed if the case vehicle had been an AT/SST. Thus, if the cargo of the case vehicle was lumber and the lumber caught on fire, the lumber fire is disregarded. On the other hand, fires involving the tractor, fuel system, brakes or any other element of the tractor or trailer (excluding cargo) of the case vehicle are coded. In addition, all fires associated with other vehicles, objects or material not part of or carried by the case vehicle are coded. A data form is filled out for each case vehicle and each applicable fire; all other information on the form is coded in relation to that specific case vehicle and fire source.

For example, consider an accident involving a collision between two tractor semi-trailers (vehicles 1 and 2). Both of their fuel tanks rupture and catch fire. The fire spreads from the fuel tank of vehicle 1 to the cargo in the trailer of vehicle 1 . The trailer and cargo of vehicle 2 are not involved in the fire. For vehicle 2, three fires are coded: the fuel tank fire of vehicle 2, the fuel tank fire of vehicle 1 , and the trailer/cargo fire of vehicle 1 . For vehicle 1 , only two fires are coded: the fuel tank fire of vehicle 2 and the fuel tank fire of vehicle 1 . The fire in the trailer/cargo of vehicle 1 is disregarded in considering the fire affecting vehicle 1 since the secure trailer will not burn in this manner.

If the only fire in the accident involved the cargo of the case vehicle, the fire source number is assigned the number 98 , meaning that the fire is not applicable. If a fire could not be identified from the police report, this field is coded 99 , meaning that the number of fires and all other information with respect to the fire environment is unknown.

Most of the remaining information required on the form is self-explanatory. If an entry is unknown, all nines are entered. For example, if the fire duration is unknown, the code 9999 is entered. The fire duration is intended to represent the time period during which an energetic open flame existed (excluding any time that the combustibles were smoldering). A brief description of the type of fire can be entered under the heading "Description". The shortest distance from the fire to the trailer of the case vehicle is based on the shortest distance from the outside surface of the trailer to the outside surface of the fire. The length and width of the fire are used to define the surface area of the fire, which represents the ground area covered by open flame. If information on the surface area of the fire is not explicitly provided but it is known that a vehicle was engulfed in fire (as stated in police reports or phone interviews), the surface area of the fire is taken to be approximately equal to the footprint of that vehicle. The walls of the trailer of the case vehicle with a direct line of sight to the fire are used to define which walls are directly heated by the fire. 
Accident Year:

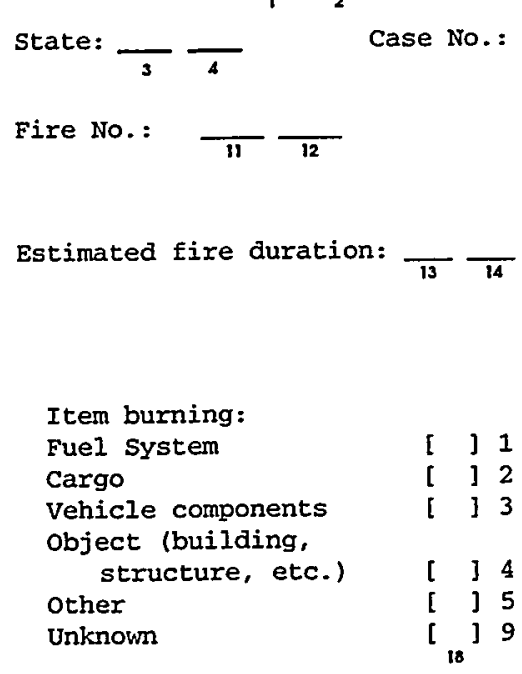

\begin{abstract}
State: $\frac{}{3} \frac{1}{4} \quad$ Case No.: $\frac{1}{5} \frac{2}{6} \frac{7}{7} \frac{1}{8} \quad$ FARS Vehicle No.: $\frac{}{9} \frac{1}{10}$
\end{abstract}
Fire No.: $\frac{}{11} \frac{}{12}$

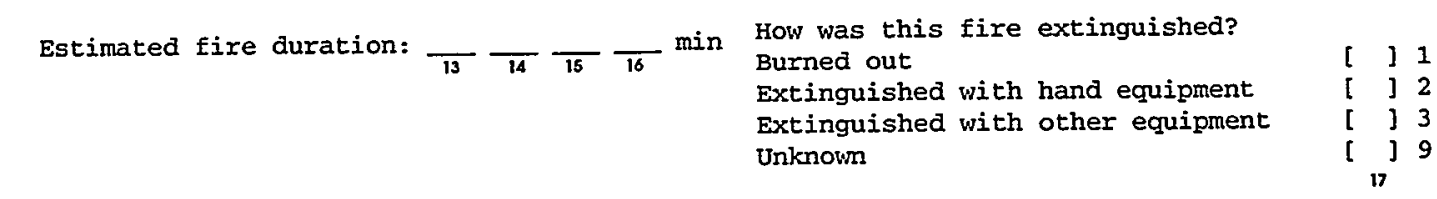

\begin{tabular}{|c|c|c|c|}
\hline Fuel system & [ ] 1 & Type of fire: & \\
\hline Cargo & [ $\quad] 2$ & Combustible gas & 【 \\
\hline Vehicle components & [ 13 & Combustible Iiquid & [ \\
\hline object (building, & & Combustible solid & {$\left[\begin{array}{ll}{[} & ]\end{array}\right.$} \\
\hline structure, etc.) & [ ] 4 & object & 1 \\
\hline Other & [ ] 5 & Otner & {$\left[\begin{array}{ll}1 \\
{[}\end{array}\right]$} \\
\hline Unknown & {$\left[{ }_{18}\right] 9$} & & 19 \\
\hline
\end{tabular}

Description: $\frac{7}{20} \frac{7}{21} \frac{7}{23} \frac{7}{24} \frac{75}{25} \frac{7}{27} \frac{-}{25} \frac{7}{29} \frac{70}{31} \frac{}{32} \frac{1}{33} \frac{}{34}$

Mass of combustible cargo/fuel: $\frac{}{35} \frac{-}{36} \frac{7}{37} \frac{7}{39}$ lbs

Shortest distance from fire to trailer of case vehicle: $\frac{}{40} \frac{1}{41} \frac{1}{42}$

- Length of fire: $\frac{}{43} \frac{1}{45} \mathrm{ft}$

Width of fire: $\frac{}{46} \frac{}{40} \frac{\text { ft }}{40}$

Walls of trailer of case vehicle

with direct line of sight to fire:

Rear: Bottom: Top:

Yes [ ] 1 Yes [ ] 1 Yes [ ] 1 Yes

No [ ] 2 No [ ] 2 No [ ] 2

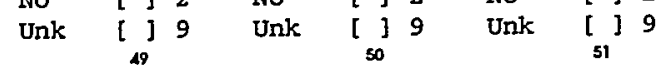

$\begin{array}{llll}\text { No } & {[} & ] & 2\end{array}$

Unk [ ] 9

\begin{tabular}{|c|c|c|c|c|}
\hline \multicolumn{3}{|c|}{ Left: } & \multicolumn{2}{|c|}{ Right: } \\
\hline Yes & [ ] & 1 & Yes & [ ] ] \\
\hline No & [ ] & 2 & No & [ ] \\
\hline Unk & []$\left._{53}\right]$ & 9 & Unk & [ ] \\
\hline
\end{tabular}

Orientation of this fire relative to closest point on trailer of case vehicle:

$$
\overline{55} \frac{0^{\prime} \mathrm{clock}}{56}
$$

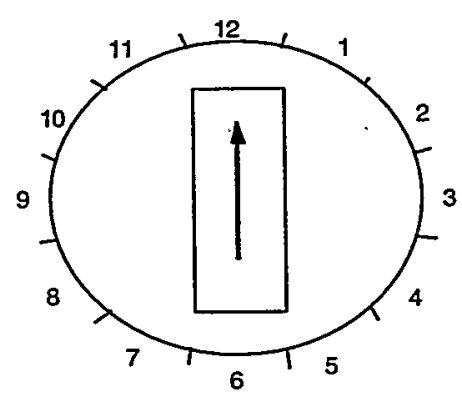

Figure 6-3 Supplemental Data Form for Fire Severity (1992-1996) 
--This page left almost blank intentionally-- 


\section{Compilation of Accident Files}

The AT/SST-surrogate accident files are created from existing accident data files and supplemental data obtained from police reports. The final accident data developed for this project are contained in several files. The first two files are derived from existing accident data files. They are used to determine frequencies of the most harmful event, impact direction, other vehicle or object involved, rollover occurrence, and fire occurrence. The other four data files were developed from the supplemental data collection efforts for collision and fire severity and are used to determine the frequency of impact location and distribution functions for peak contact velocity, fire separation, fire size and fire duration. The impact severity studies focused on most harmful events that posed the most serious threat to the AT/SST and its cargoes. The fire data were collected on all types of accidents in which fire occurred.

\subsection{Main Accident File Constructed from TIFA}

This file is a subset of the 1992-1996 TIFA file. Only tractor semi-trailers are included in the file (the TIFA file includes all medium and heavy duty trucks). The file includes forty-five variables from the TIFA file and three variables generated for this study. The TIFA file variables include case identifiers and variables on the accident environment, the events of the accident, operator of the truck and the physical configuration of the truck. The added variables code the type of other vehicle involved in the accident, whether any truck in the accident suffered a fire, and the event tree category for the accident. A list of variables in this file appears in Table 7-1. The file has 9,220 records representing 13,522 vehicles. The file does not include all TIFA variables, nor does it include all TIFA records.

The coding of those variables copied directly from the TIFA database can be found in Reference 16. The coding of the three variables created for this study is described here. The 'other vehicle type' variable classifies the accident the case vehicle was involved in according to the following rules:

- Code level 0 is used if there is no other vehicle involved in the accident (i.e., the accident is a single-vehicle accident);

- Code level 1 is used if there is one other truck, no cars, and the other truck is not a hazardous tanker; or if there are two or more other trucks involved and neither is a hazardous tanker;

- Code level 2 applies if there is any number of cars and no trucks; or if there is one other truck (not a hazardous tanker) and any number of cars; and

- Code level 3 is used for any accident in which any of the other vehicles involved is a hazardous tanker. 
Table 7-1 List of Variables in Main Accident File

\begin{tabular}{|c|l|c|l|}
\hline $\begin{array}{c}\text { TIFA } \\
\text { Variable No. }\end{array}$ & Description & $\begin{array}{c}\text { TIFA } \\
\text { Variable No. }\end{array}$ & Description \\
\hline 11 & Year & 140 & Fire \\
\hline 1 & State & 144 & Most harmful event \\
\hline 2 & FARS number & 162 & $1^{\text {st }}$ vehicle related factor \\
\hline 104 & Vehicle number & 163 & $2^{\text {nd }}$ vehicle related factor \\
\hline 12 & Hour & 227 & $1^{\text {st }}$ driver related factor \\
\hline 14 & No. of vehicles & 228 & $2^{\text {nd }}$ driver related factor \\
\hline 16 & Land use & 229 & $3^{\text {rd }}$ driver related factor \\
\hline 18 & Roadway function class & 308 & Driver age \\
\hline 19 & Route signing & 1010 & Power unit axles \\
\hline 23 & $1^{\text {st }}$ harmful event & 1012 & Power unit length \\
\hline 24 & Manner of collision & 1018 & Trailer axles \\
\hline 29 & Speed limit & 1021 & Trailer cargo weight \\
\hline 31 & Roadway profile & 1022 & Trailer length \\
\hline 33 & Roadway condition & 1023 & Trailer cargo body \\
\hline 37 & Light condition & 1026 & Trailer hazardous cargo \\
\hline 38 & Weather & 1048 & Combination code \\
\hline 51 & No. of drinking drivers & 1052 & Gross combination \\
& & & weight \\
\hline 125 & Rollover & 1056 & Cargo spillage \\
\hline 127 & Travel speed & 1057 & Area of operation \\
\hline 128 & Hazardous cargo & 1058 & Operating authority \\
\hline 135 & Initial impact & 1076 & Sample weight \\
\hline 136 & Principal impact & 2011 & Other vehicle type \\
\hline 137 & Extent of deformation & 2012 & Fire on any truck \\
\hline 138 & Vehicle role & 2500 & Event tree \\
\hline & & & \\
\hline
\end{tabular}

The 'other vehicle type' field was generated in two steps. In the first step, variables containing counts of vehicle types involved in the accident were generated. Variable v2001 contained a count of the number of other trucks, buses, and fire trucks involved in the accident. Variable v2002 contained the number of passenger cars, motorcycles, and light trucks. Variable v2006 contained the number of tank trucks carrying hazardous commodities. Variable 2001 was generated from FARS using the 'body type' variable(v110). If v110 was coded 40, 41, 48, 49, $60,61,62,63,64,66,67,68,69,70,71,72,78$, or 79 , or the gross vehicle weight rating (GVWR) was class 3 through class 8 , then the vehicle was counted as a truck. Variable 2002 was also generated from FARS using the 'body type' variable. If variable 110 was coded 1-49, 80-91, or 97-99, and GVWR was not class 3 through 8 then the vehicle was counted as a car. 
Variable 2006 was coded from the TIFA variables for 'straight truck body style' (variable 1008), 'straight truck hazardous cargo' (variable 1016), 'first trailer body style' (variable 1023), and 'first trailer hazardous cargo' (variable 1026). If (v1008=tank and v1016=yes) or (v1023 =tank and v1026=yes) then the vehicle was counted as a hazardous tank.

With these variables, the following logic was used to generate the 'other vehicle type' field:

if $\mathrm{v} 2001=0$ and $\mathrm{v} 2002=0$ and $\mathrm{v} 2006=0$ then $\mathrm{v} 2011=0$

if (v2001>=1 and v2002=0 and v2006=0) or (v2001>=2 and v2006=0) then v2011=1

if ( $\mathrm{v} 2001=0$ or $\mathrm{v} 2001=1)$ and $\mathrm{v} 2006=0$ and $\mathrm{v} 2002>=1$ then $\mathrm{v} 2011=2$

if $\mathrm{v} 2006>=1$ and $\mathrm{v} 1026 \diamond 1$ then $\mathrm{v} 2011=3$

The 'fire on any truck' field records whether any truck in the accident is coded as having a fire. Even if the case vehicle did not have a fire, if another truck in the accident had a fire this field was coded yes (code level 1). If none of the trucks involved in the accident, including the case vehicle, experienced a fire, this field was coded no (code level 0 ). To produce this field, a variable (v2012) was generated in the TIFA file, using variable 140 ('fire occurrence'), to count the number of trucks involved in the accident which had a fire. The 'fire on any truck' field was then produced as follows:

if $\mathrm{v} 2012=0$ then [fire on any truck] $=$ no

if $v 2012>=1$ then [fire on any truck] $=$ yes

The 'event tree' field is developed based on TIFA Variable 144 (most harmful event) but includes some regrouping to improve differentiation of accident severities. The code levels are:

1. most harmful event is collision with heavy or medium truck (not a tanker)

2. most harmful event is collision with a light truck or car

3. most harmful event is collision with a tanker

4. most harmful event is collision with a high, hard object

5. most harmful event is collision with a high soft object

6. most harmful event is collision with an angled, hard object

7. most harmful event is collision with an angled, soft object

8. most harmful event is collision with a low, hard object

9. most harmful event is collision with a low, soft object

10. most harmful event is collision with a non-fixed object

11. most harmful event is collision with a train

12. most harmful event is rollover

13. most harmful event is fire

14. most harmful event is tank fire

15. most harmful event is immersion

16. most harmful event is other non-collision event most harmful event is unknown (blank) 
The 'other vehicle type' field (v2011) was used in combination with the TIFA variable for most harmful event to produce the 'event tree' according to the following logic:

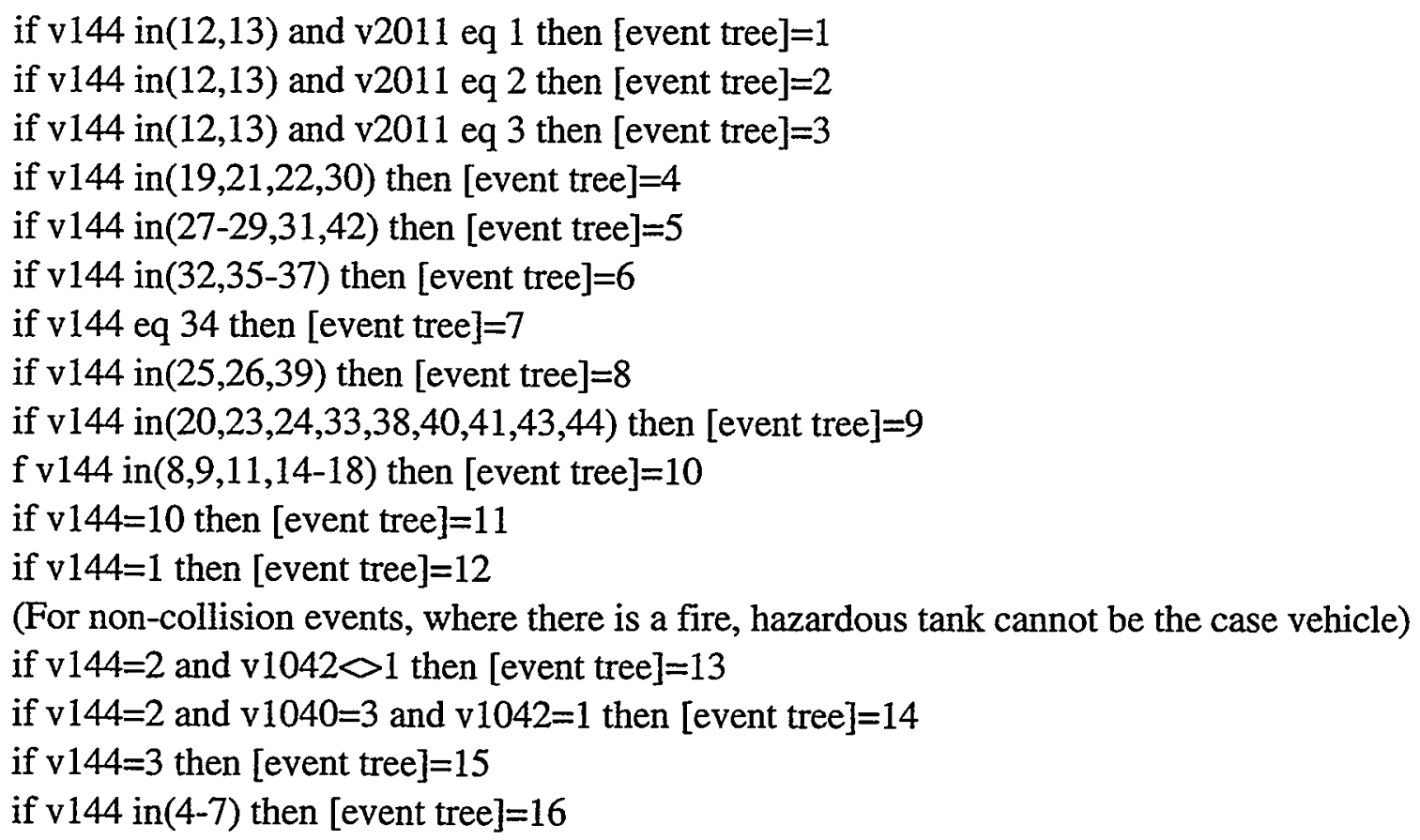

\subsection{Accident File Constructed from GES Data}

This file is a subset of the 1992-96 GES file. Only tractor semi-trailers are included in the file. All variables from the accident and vehicle GES files are included, as well as five additional variables generated for this study. Two variables code the number of trucks and the number of cars involved in the accident. Another records the vehicle type class of the accident. There is also a variable which codes whether any vehicle in the accident was towed and another variable coding the event tree category for the accident. The full list of variables appear in Table 7-2. The file has 24,355 records representing 891,190 vehicles.

Variables 2001, 2002, 2011, 2013, and 2500 were generated from the GES file. Variables 2001 and 2002 record the number of trucks and cars, respectively, involved in the accident. Trucks are identified using the body-type variable (v112) in GES. If v112 in(50,58, $59,60,64,65,66,78,92,93)$, then the vehicle was identified as a truck. If v112 in(1-49,91,97-99), then the vehicle was identified as a car.

Variable 2011 has three code levels:

- Code level 0 is used if there is no other vehicle involved in the accident (i.e., the accident is a single-vehicle accident); 
Table 7-2 List of Variables in Accident File Constructed from GES

\begin{tabular}{|c|l|c|l|}
\hline Variable & Description & Variable & Description \\
\hline V1 & Case & V104 & Violations charged \\
\hline V2 & Month & V105 & Driver vision obscured \\
\hline V3 & Year & V106 & Driver avoid maneuver \\
\hline V4 & Day & V108 & Hit and run \\
\hline V5 & Hour & V109 & Vehicle make \\
\hline V6 & Minute & V110 & Vehicle model \\
\hline V7 & Number vehicles & V111 & Model year \\
\hline V8 & Number non-motorists & V112 & Body type \\
\hline V9 & Land use & V115 & VIN \\
\hline V10 & Percent rural & V116 & Special use \\
\hline V11 & First harmful & V120 & Emergency use \\
\hline V12 & Manner collision & V122 & Travel speed \\
\hline V13 & Interstate & V123 & Vehicle defects \\
\hline V14 & Relation to junction & V124 & Vehicle trailing \\
\hline V15 & Relation to roadway & V125 & Jackknife \\
\hline V16 & Traffic way flow & V126 & Rollover \\
\hline V17 & Number lanes & V127 & Fire \\
\hline V18 & Roadway alignment & V129 & Damage area \\
\hline V19 & Roadway profile & V134 & Damage severity \\
\hline V20 & Roadway surface & V135 & Manner of leaving \\
\hline V21 & Traffic control & V136 & Most harmful \\
\hline V22 & Speed limit & V137 & Vehicle maneuver \\
\hline V23 & Light condition & V142 & Vehicle role \\
\hline V24 & Weather & V143 & Accident type \\
\hline V25 & School bus related & V144 & Maximum injury vehicle \\
\hline V26 & Ped/cycle type & V145 & Number injured vehicle \\
\hline V27 & Maximum injury accident & V146 & Driver drinking \\
\hline V28 & Number injured accident & V152 & Weight \\
\hline V29 & Alcohol involved accident & V2001 & Number of trucks \\
\hline V32 & PSU & V2002 & Number of cars \\
\hline V33 & Census region & V2011 & Vehicle type \\
\hline V102 & Coded vehicle number & V2013 & Vehicle towed \\
\hline V103 & Driver presence & V2500 & Event tree \\
\hline & & & \\
\hline & & \\
\hline
\end{tabular}


- Code level 1 is used if there is one other truck, and no cars; or if there are two or more other trucks involved;

- Code level 2 applies if there is any number of cars and no trucks; or if there is one other truck and any number of cars.

The following logic is used to generate variable 2011:

$$
\begin{aligned}
& \text { if } \mathrm{v} 2001=0 \text { and } \mathrm{v} 2002=0 \text { then } \mathrm{v} 2011=0 \\
& \text { if (v2001=1 and } \mathrm{v} 2002=0 \text { ) or } \mathrm{v} 2002>=2 \text { then } \mathrm{v} 2011=1 \\
& \text { if ( } 2001=0 \text { and } \mathrm{v} 2002<=1) \text { or }(\mathrm{v} 2001=1 \text { and } \mathrm{v} 2002<=1) \text { then } \mathrm{v} 2001=2
\end{aligned}
$$

The 'vehicle towed' variable (2013) shows whether any vehicle in the accident was towed. This was generated from variable 135 (manner of leaving scene) in the GES file which records how a particular vehicle left the accident scene. Code level 2 of variable 135 records whether the vehicle was towed due to damage; code level 3 records whether the vehicle was towed for some other reason. Variable 2013 was generated by checking if any vehicle in the accident was coded 2 or 3 for variable 135 .

The 'event tree' field, variable 2500, was based on variable 136 (most harmful event) and on the UMTRI-generated 'vehicle type' variable. The code levels are:

1 most harmful event is collision with heavy or medium truck

2 most harmful event is collision with a light truck or car

3 most harmful event is collision with a high, hard object

4 most harmful event is collision with a high soft object

5 most harmful event is collision with an angled, hard object

6 most harmful event is collision with a low, hard object

7 most harmful event is collision with a low, soft object

8 most harmful event is collision with an other fixed object

9 most harmful event is collision with a non-fixed object

10 most harmful event is collision with a train

11 most harmful event is rollover

12 most harmful event is fire

13 most harmful event is immersion

14 most harmful event is other non-collision event most harmful event is unknown (blank)

The following logic was used to generate the 'event tree' variable in the GES file:

if $\mathrm{v} 136=25$ and $\mathrm{v} 2011=1$ then [event tree] $=1$

if $v 136=25$ and $v 2011=2$ then [event tree] $=2$

if v136 in $(32,34)$ then [event tree] $=3$

if $\mathrm{v} 136$ in $(37,45)$ then [event tree] $=4$ 
if $\mathrm{v} 136 \mathrm{in}(38,40)$ then [event tree] $=5$

if $v 136$ in $(36,42)$ then [event tree] $=6$

if v136 in $(31,33,35,39,41,43,44,58)$ then [event tree] $=7$

if v136 eq 59 then [event tree] $=8$

if $\mathrm{v} 136$ in $(21,22,24,26-29,46)$ then [event tree] $=9$

if $v 136$ eq 23 then [event tree] $=10$

if $v 136$ eq 1 then [event tree] $=11$

if v136 eq 2 then [event tree] $=12$

if $v 136$ eq 3 then [event tree] $=13$

if v136 in(4-10) then [event tree] $=14$

\subsection{Fire Severity Data - TIFA}

This file contains supplementary data on the severity of fires in accidents involving AT/SST surrogates. Supplementary data were collected for all cases in which a surrogate vehicle was involved and at least one truck in the accident was coded as having a fire for data years 19921996. A total of 490 cases and 727 fires were coded. The variables in this file are listed in Table 7-3. A more detailed discussion of the variable listed in Table 7-3 can be found in Section 6.2.

Table 7-3 List of Variables in the TIFA Fire Severity File

\begin{tabular}{|c|l|c|l|}
\hline $\begin{array}{c}\text { TIFA } \\
\text { Variable No. }\end{array}$ & Description & $\begin{array}{c}\text { TIFA } \\
\text { Variable No. }\end{array}$ & Description \\
\hline 11 & Year & NA & Standoff distance \\
\hline 1 & State & NA & Length of fire \\
\hline 2 & FARS number & NA & Width of fire \\
\hline 104 & Vehicle number & NA & Front in line of sight to fire \\
\hline NA & Fire number & NA & Rear in line of sight to fire \\
\hline NA & Fire duration & NA & Bottom in line of sight to fire \\
\hline NA & How extinguished & NA & Top in line of sight to fire \\
\hline NA & Item burning & NA & Left in line of sight to fire \\
\hline NA & Type of fire & NA & Right in line of sight to fire \\
\hline NA & Description & NA & Orientation to trailer \\
\hline NA & Cargo weight & NA & Comments \\
\hline NA & Precision of estimate & 1076 & Sample weight \\
\hline
\end{tabular}




\subsection{Collision Severity Data - TIFA}

The focus of the data collected for the collision severity data file was on those accidents that pose the greatest threat to the integrity of the SST and its cargoes. The file contains data corresponding to twelve different types of accidents:

1. The most harmful event is a collision with a medium or heavy truck;

2. The most harmful event is a collision with a car or light truck;

3. The most harmful event is involvement with a hazardous tanker;

4. The most harmful event is a collision with a fixed object (both hard and soft);

5. The most harmful event is a collision with a non-fixed object;

6. The most harmful event is a collision with a train;

7. The most harmful event is a rollover;

8. The most harmful event is a fire;

9. The most harmful event is a fire and the case vehicle is a hazardous tanker;

10. The most harmful event is immersion;

11. The most harmful is other;

12. The most harmful event is unknown.

Data were not collected for accident types which either do not involve a collision or in which the collision is unlikely to threaten the integrity of the SST and its cargo. For example, severity data for cases for which the most harmful event is a collision with a non-fixed object (e.g., pedestrians, bicyclist and animals) were not obtained.

Cases in the categories listed above were selected by different means, depending on the exigencies of the particular category, although there were some general considerations. Estimating collision severity requires estimates of travel speed, impact speed and/or skid distances. Some states do not report this information and in others reporting is spotty. Overall, travel speed is not recorded for about $50 \%$ of the cases in the TIFA file. Missing data rates for travel speed were examined on a state-by-state basis; only twenty-one states had reporting rates greater than $60 \%$. Table $7-4$ is a list of these state names. Except where noted, the cases for which collision severity data were generated come from these twenty-one states. Limiting the number of states considered reduced the level of effort required to generate the supplemental data on collision severity. The twenty-one states represent a reasonable geographic distribution for the continental U.S. Over $48 \%$ of all TIFA cases are from these states.

A total of 308 records were developed for TIFA cases in which the most harmful event was collision with a medium or heavy truck. This includes all TIFA cases in which the most harmful event was collision with a medium or heavy truck from 1992-1996 in the twenty-one states listed in Table $7-4$. 
Table 7-4 States Selected for Review of Collision Severity Data

\begin{tabular}{|llll|}
\hline Alabama & Louisiana & Ohio & Virginia \\
Arizona & Minnesota & Oklahoma & Wisconsin \\
California & Missouri & Pennsylvania & Wyoming \\
Colorado & Montana & South Carolina & \\
Delaware & Nevada & Utah & \\
Florida & North Carolina & Vermont & \\
\hline
\end{tabular}

A total of 286 records were developed for TIFA cases in which the most harmful event was collision with a car or light truck. Because of the large number of TIFA cases in which the most harmful event is collision with a car or light truck (3,241 in the period 1992-1996 in the twenty-one states with high reporting rates for travel speed), it was not feasible to review all cases. Moreover, it was unnecessary to review large numbers of cases, since this type of accident is not likely to seriously threaten the integrity of an SST or its cargo. Thus, approximately 50 cases were selected randomly from each of five different collision orientations: head-on, truck side-on into car, car side-on into truck, truck rear-end into car, and car rear-end into truck.

A total of 7 records were developed for TIFA cases in which the most harmful event was involvement with a hazardous tanker. This includes all TIFA cases in which the most harmful event was involvement with a hazardous tanker from 1992-1996 in the twenty-one states listed in Table 7-4.

A total of 239 records were generated for TIFA cases in which the most harmful event was collision with a fixed object. All cases meeting these criteria in the twenty-one states with high reporting rates for travel speed for the period 1992-1996 were included.

A total of 107 records were generated for TIFA cases in which the most harmful event was collision with a non-fixed object. For reasons similar to those given above for cases involving collisions with cars or light trucks, approximately 20 cases were selected from each data year.

All available TIFA cases (51) in which the most harmful event was collision with a train for the period 1992-1996, regardless of state, were reviewed and are included in the collision severity data file.

A total of 322 records were developed for TIFA cases in which the most harmful event was rollover. All cases meeting these criteria in the twenty-one states with high reporting rates for travel speed for the period 1992-1996 were included.

A total of 78 records were developed for TIFA cases in which the most harmful event was fire. All cases meeting these criteria in the twenty-one states with high reporting rates for travel speed for the period 1992-1996 were included. 
A total of 18 records were developed for TIFA cases in which the most harmful event was fire and the case vehicle was a hazardous tanker. All cases meeting these criteria in the twenty-one states with high reporting rates for travel speed for the period 1992-1996 were included.

A total of 7 records were developed for TIFA cases in which the most harmful event was immersion. All cases meeting these criteria in the twenty-one states with high reporting rates for travel speed for the period 1992-1996 were included.

A total of 7 records were developed for TIFA cases in which the most harmful event was other non-collision. All cases meeting these criteria in the twenty-one states with high reporting rates for travel speed for the period 1992-1996 were included.

A total of 13 records were developed for TIFA cases in which the most harmful event was unknown. All cases meeting these criteria in the twenty-one states with high reporting rates for travel speed for the period 1992-1996 were included.

The total number of records in the TIFA collision severity data file is 1455 .

Table 7-5 is a list of the variables in the TIFA collision severity data file. These data were exported from the spreadsheet described in Section 6.1 and imported into a Paradox database. A more detailed discussion of the variables listed in Table 7-5 can be found in Section 6.1.

Table 7-5 List of Variables in the TIFA Collision Severity Data File

\begin{tabular}{|c|l|c|l|}
\hline $\begin{array}{c}\text { TIFA } \\
\text { Variable No. }\end{array}$ & Description & $\begin{array}{c}\text { TIFA } \\
\text { Variable No. }\end{array}$ & Description \\
\hline 11 & Year & NA & Other travel speed \\
\hline 1 & State & NA & Other skid \\
\hline 2 & FARS number & NA & Other impact \\
\hline 104 & Vehicle number & NA & Other weight \\
\hline NA & Travel speed & NA & Area struck on tractor \\
\hline NA & Skid distance & NA & Area struck on trailer \\
\hline NA & Friction coefficient & NA & Mass elements \\
\hline NA & Impact & NA & Object struck \\
\hline NA & Angle & NA & Road surface \\
\hline NA & Rel. velocity (best estimate) & NA & Accident event tree \\
\hline NA & Weight & 1076 & Sample weight \\
\hline NA & Peak contact velocity & & \\
\hline
\end{tabular}




\section{Discussion and Analysis of Accident Types}

The accident types used in the DPTRA study are defined by (1) the most harmful event (MHE), ${ }^{11}$ (2) the impact direction, (3) the type of the other vehicle or object involved, (4) the impact location, (5) rollover occurrence and (6) fire occurrence. The following sections provide a discussion of these six accident factors.

\subsection{MHE}

In the risk assessment for the DPTRA study, accidents are initially characterized by the MHE. The definition is based on the event tree variable in the main accident file constructed from the TIFA file, as indicated in Table 8-1. The MHE is used to differentiate accident events that have different accident severities, consistent with the information available in the databases. The key factors considered in selecting the MHEs used in the risk assessment were weight, crush characteristics and fuel capacity.

Table 8-1 Definition of MHE

\begin{tabular}{|l|l|}
\hline MHE & Description \\
\hline 1. Truck & Collision with medium or heavy truck (event tree $=1)$ \\
\hline 2. Car & Collision with light truck or car (event tree $=$ 2) \\
\hline 3. Tanker & Involvement with hazardous tanker (event tree=3 or 14) \\
\hline 4. Hard Object & Collision with hard, fixed object (event tree=4 or 6 or 8 ) \\
\hline 5. Soft Object & Collision with soft, fixed object (event tree=5 or 7 or 9$)$ \\
\hline 6. Non-fixed Object & Collision with non-fixed object (event tree $=10)$ \\
\hline 7. Train & Collision with train (event tree $=11)$ \\
\hline 8. Rollover & Rollover (event tree $=12)$ \\
\hline 9. Fire & Fire (event tree $=13)$ \\
\hline 10. Immersion & Immersion (event tree $=15)$ \\
\hline
\end{tabular}

Note that fires and rollover can occur even when the MHE is a collision event and collisions may occur when the MHE is fire, rollover or immersion. These events are not mutually exclusive. Subsequent factors are used to evaluate the probability of multiple threats occurring in the same accident. This structure introduces some redundancy but is required by limitations of the databases used to quantify the questions. In the TIFA and GES databases, the MHE is

\footnotetext{
${ }^{11}$ Most harmful event as used in the risk assessment for the DPTRA study should not to be confused with variables in the accident data files by the same name. The acronym for most harmful event will only be used in the context applicable to risk assessment and/or the DPTRA study.
} 
generally coded based on the environment that is most harmful to vehicle occupants and/or pedestrians. There is no attempt to describe the sequence of accident events in these databases.

Table 8-2 shows the weighted number of fatal involvements by MHE and operating environment as determined from the main accident file constructed from the TIFA file. Four categories of operating environment are considered: limited access roads in urban population areas, limited access roads in rural population areas, other roads in urban population areas and other roads in rural population areas. TFA variables 16 (land use), 18 (roadway function class) and 19 (route signing) were used to define the operating environment based on the following logic:

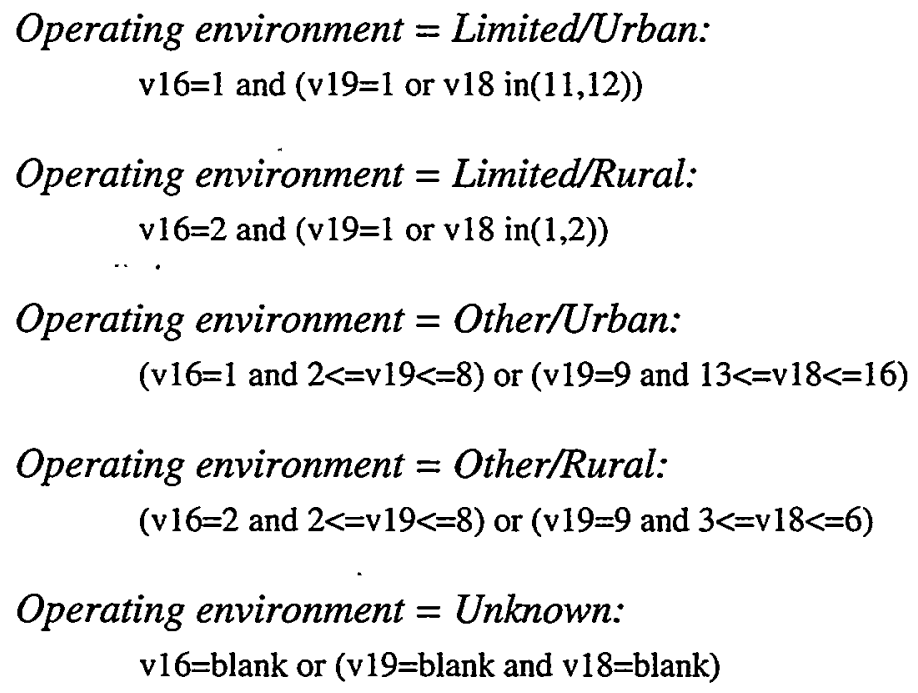

Table 8-3 indicates the frequency with which each MHE occurs given a fatal accident in the indicated operating environment. There are some significant variations in the frequencies of MHEs with the operating environment, notably for train collisions, which almost never occur on limited access roadways.

Table 8-4 provides a comparison of the overall frequencies of MHE based on TIFA data and GES data for different accident severity levels (i.e., all; fatal or tow-away; and fatal). The GES data do not allow hazardous tankers to be explicitly identified and so collisions with trucks and hazardous tanker involvements are combined into the truck category in Table 8-4. Furthermore, immersion events are not explicitly identified in GES but there is an 'other noncollision' category in GES, so that is used in place of immersion in Table 8-4. Examination of Table 8-3 and Table 8-4 shows that the overall frequencies of MHE are in general less dependent on the accident severity level than on the operating environment. Therefore, use of the fatal accident data to evaluate the frequencies of MHE does not introduce any significant error. 
Table 8-2 Weighted Number of Fatal Accidents by MHE and Operating Environment

\begin{tabular}{|l|r|r|r|r|r|r|}
\hline MHE & $\begin{array}{c}\text { Limited } \\
\text { Urban }\end{array}$ & $\begin{array}{c}\text { Limited } \\
\text { Rural }\end{array}$ & $\begin{array}{c}\text { Other } \\
\text { Urban }\end{array}$ & $\begin{array}{c}\text { Other } \\
\text { Rural }\end{array}$ & Unknown & Total \\
\hline Truck & 147 & 520 & 46 & 153 & 10 & 876 \\
\hline Car & 1293 & 3963 & 1691 & 2758 & 141 & 9846 \\
\hline Tanker & 2 & 13 & 1 & 4 & 0 & 20 \\
\hline Hard Object & 58 & 119 & 11 & 39 & 1 & 228 \\
\hline Soft Object & 31 & 121 & 18 & 54 & 1 & 225 \\
\hline Non-Fixed Object & 258 & 399 & 330 & 134 & 14 & 1135 \\
\hline Train & 0 & 6 & 18 & 47 & 1 & 72 \\
\hline Rollover & 100 & 340 & 36 & 296 & 8 & 780 \\
\hline Fire & 24 & 121 & 5 & 35 & 3 & 188 \\
\hline Immersion & 1 & 8 & 0 & 7 & 0 & 16 \\
\hline Subtotal & 1914 & 5610 & 2156 & 3527 & 179 & 13386 \\
\hline Unknown & 37 & 48 & 15 & 33 & 3 & 136 \\
\hline Total & 1951 & 5658 & 2171 & 3560 & 182 & 13522 \\
\hline
\end{tabular}

Table 8-3 Frequency of MHE by Operating Environment (Fatal Accidents)

\begin{tabular}{|l|r|r|r|r|}
\hline MHE & $\begin{array}{c}\text { Limited } \\
\text { Urban }\end{array}$ & $\begin{array}{c}\text { Limited } \\
\text { Rural }\end{array}$ & $\begin{array}{c}\text { Other } \\
\text { Urban }\end{array}$ & \multicolumn{1}{c|}{$\begin{array}{c}\text { Other } \\
\text { Rural }\end{array}$} \\
\hline Truck & $7.68 \%$ & $9.27 \%$ & $2.13 \%$ & $4.34 \%$ \\
\hline Car & $67.55 \%$ & $70.64 \%$ & $78.43 \%$ & $78.20 \%$ \\
\hline Tanker & $0.10 \%$ & $0.23 \%$ & $0.05 \%$ & $0.11 \%$ \\
\hline Hard Object & $3.03 \%$ & $2.12 \%$ & $0.51 \%$ & $1.11 \%$ \\
\hline Soft Object & $1.62 \%$ & $2.16 \%$ & $0.83 \%$ & $1.53 \%$ \\
\hline Non-Fixed Object & $13.48 \%$ & $7.11 \%$ & $15.31 \%$ & $3.80 \%$ \\
\hline Train & $0.00 \%$ & $0.11 \%$ & $0.83 \%$ & $1.33 \%$ \\
\hline Rollover & $5.22 \%$ & $6.06 \%$ & $1.67 \%$ & $8.39 \%$ \\
\hline Fire & $1.25 \%$ & $2.16 \%$ & $0.23 \%$ & $0.99 \%$ \\
\hline Immersion & $0.05 \%$ & $0.14 \%$ & $0.00 \%$ & $0.20 \%$ \\
\hline
\end{tabular}

\subsection{Impact Direction}

The impact direction defines the principal component of deceleration, i.e., positive or negative longitudinal, or lateral. The impact direction is an important accident characteristic for the AT/SST because the failure thresholds for the cargo tie-down system depend on the principal direction of deceleration. The impact direction for accidents involving collision angles between $-30^{\circ}$ and $30^{\circ}$ (where $0^{\circ}$ is head-on) were classified as front, for which the principal 
component of deceleration is assumed to be in the positive longitudinal direction. The impact direction for accidents involving collision angles between $150^{\circ}$ and $210^{\circ}$ were classified as rear, for which the principal component of deceleration is assumed to be in the positive longitudinal direction. All other impacts were classified as side, for which the principal component of deceleration is assumed to be lateral. Because the strength of the AT/SST tiedown system in the longitudinal direction is more than twice that in the lateral direction, this classification results in a conservative treatment of tie-down system failure. Since some accidents do not involve a collision, non-collision events (in which the deceleration of the case vehicle is assumed to be small) must also be considered.

Table 8-4 Overall Frequency of each MHE for Different Accident Thresholds

\begin{tabular}{|l|r|r|r|r|}
\hline MHE & GES-All & $\begin{array}{c}\text { GES-Fatal or } \\
\text { Tow-away }\end{array}$ & TIFA-Fatal & GES-Fatal \\
\hline Truck (including Tanker) & $7.30 \%$ & $8.51 \%$ & $6.67 \%$ & $6.15 \%$ \\
\hline Car & $68.53 \%$ & $71.15 \%$ & $73.30 \%$ & $76.74 \%$ \\
\hline Hard object & $2.15 \%$ & $2.36 \%$ & $1.70 \%$ & $0.23 \%$ \\
\hline Soft object & $7.49 \%$ & $3.20 \%$ & $1.67 \%$ & $1.81 \%$ \\
\hline Non-fixed object & $5.75 \%$ & $1.80 \%$ & $8.45 \%$ & $8.73 \%$ \\
\hline Train & $0.16 \%$ & $0.34 \%$ & $0.54 \%$ & $0.44 \%$ \\
\hline Rollover & $2.95 \%$ & $7.18 \%$ & $5.81 \%$ & $4.61 \%$ \\
\hline Fire & $0.21 \%$ & $0.38 \%$ & $1.40 \%$ & $0.88 \%$ \\
\hline Other non-collision & $5.45 \%$ & $5.07 \%$ & $0.47 \%$ & $0.41 \%$ \\
\hline
\end{tabular}

TIFA variables 135 (impact point - initial) and 136 (impact point - principal) were used to define the impact direction based on the following logic:

Impact direction=Front/rear:

$(\mathrm{v} 136=1,5,6,7,11$ or 12$)$ or ((v136=blank, 15 or 16$)$ and $(v 135=1,5,6,7,11$ or 12$))$

Impact direction $=$ Side:

$(v 136=2,3,4,8,9,10,13$ or 14$)$ or $((v 136=$ blank, 15 or 16$)$ and $(v 135=2,3,4,8,9,10,13$ or 14))

Impact direction=Non-collision:

$(v 136=0)$ or $((v 136=b l a n k, 15$ or 16$)$ and $(v 135=0))$

Impact direction=Unknown:

(v136=blank, 15 or 16$)$ and (v136=blank, 15 or 16$)$

Table 8-5 lists the number of fatal involvements by impact direction and MHE as obtained from TIFA data between 1992 and 1996. Table 8-6 lists the frequency of each category of impact direction conditional on an accident in which the MHE is of the type indicated in the first column. For six of the ten MHEs, non-collision is precluded because the accident 
involves a collision by definition of the MHE. These cases are indicated by na (not applicable) in the column listing the frequency of non-collision.

Table 8-5 Weighted Number of Fatal Accidents by Impact Direction and MHE

\begin{tabular}{|l|r|r|r|r|r|r|r|}
\hline MHE & Front & \multicolumn{1}{|c|}{ Side } & \multicolumn{1}{c|}{ Rear } & Non-collision & Subtotal & Unknown & Total \\
\hline Truck & 468 & 150 & 239 & 0 & 857 & 19 & 876 \\
\hline Car & 5818 & 2116 & 1827 & 0 & 9761 & 85 & 9846 \\
\hline Tanker & 8 & 8 & 4 & 0 & 20 & 0 & 20 \\
\hline Hard Object & 189 & 35 & 2 & 0 & 226 & 2 & 228 \\
\hline Soft Object & 172 & 40 & 4 & 0 & 216 & 9 & 225 \\
\hline Non-Fixed & 693 & 319 & 77 & 2 & 1091 & 44 & 1135 \\
\hline Train & 34 & 33 & 2 & 0 & 69 & 3 & 72 \\
\hline Rollover & 299 & 189 & 21 & 256 & 765 & 15 & 780 \\
\hline Fire & 132 & 22 & 12 & 15 & 181 & 7 & 188 \\
\hline Immersion & 9 & 3 & 0 & 2 & 14 & 2 & 16 \\
\hline Unknown & 42 & 19 & 10 & 54 & 125 & 11 & 136 \\
\hline Total & 7864 & 2934 & 2198 & 329 & 13325 & 197 & 13522 \\
\hline
\end{tabular}

Table 8-6 Frequency of Impact Direction by MHE (Fatal Accidents)

\begin{tabular}{|l|c|c|c|c|}
\hline MHE & Front & Side & Rear & Non-Collision \\
\hline Truck & $54.61 \%$ & $17.50 \%$ & $27.89 \%$ & na \\
\hline Car & $59.60 \%$ & $21.68 \%$ & $18.72 \%$ & na \\
\hline Tanker & $40.00 \%$ & $40.00 \%$ & $20.00 \%$ & $0.00 \%$ \\
\hline Hard Object & $83.63 \%$ & $15.49 \%$ & $0.88 \%$ & na \\
\hline Soft Object & $79.63 \%$ & $18.52 \%$ & $1.85 \%$ & na \\
\hline Non-Fixed & $63.52 \%$ & $29.24 \%$ & $7.06 \%$ & $0.18 \%$ \\
\hline Train & $49.28 \%$ & $47.83 \%$ & $2.90 \%$ & na \\
\hline Rollover & $39.08 \%$ & $24.71 \%$ & $2.75 \%$ & $33.46 \%$ \\
\hline Fire & $72.93 \%$ & $12.15 \%$ & $6.63 \%$ & $8.29 \%$ \\
\hline Immersion & $64.29 \%$ & $21.43 \%$ & $0.00 \%$ & $14.29 \%$ \\
\hline
\end{tabular}

The GES data do not include variables that are directly comparable to those used to define impact direction in the TIFA data. For the GES data, variables 12 (manner of collision) and 142 (damage area) were used to determine the impact direction using the following logic:

\section{Impact direction $=$ Front/rear:}

(v12 $=1,2,3,5$ or 6$)$ or $((v 12=0,8$ or 9$)$ and $(v 142=1$ or 4$))$ 
Impact direction=Side:

$v 12=4$ or $((v 12=0,8$ or 9$)$ and $(v 142=2$ or 3$))$

Impact direction $=$ Non-collision:

(v12=0, 8 or 9$)$ and $v 142=0$

Impact direction $=$ Unknown:

(v12=0, 8 or 9$)$ and (v142=5, 6, 8 or 9$)$

Table 8-7 shows a comparison the overall frequencies of impact direction by MHE based on TIFA data and GES data for different accident severity levels (i.e., all, fatal or tow-away, and fatal). The variation with the database used and the reporting thresholds are significant in the case of impact direction. As the accident severity level becomes more severe, the estimates for the frequencies of impact direction by MHE obtained with the GES data come closer to those obtained with the TIFA data. Nevertheless, the estimates for the frequencies of impact direction by MHE made with TIFA and those made with GES, regardless of the severity level, are considerably different. The estimates obtained with GES suffer from two problems: (1) the sample sizes are small in many of the cells of this two-way distribution, particularly for fatal accidents, (2) the variables used to define the impact direction for the GES estimates are not as descriptive of the impact location as those used for the TIFA estimates. TIFA provides a much larger sample and variables that can be used to obtain a more precise description of the impact direction than those in GES. For these reasons, the estimated frequencies of impact direction by MHE from TIFA are considered to be more representative and more accurate than those from GES.

\subsection{Other Vehicle Involved}

For cases in which the MHE is coded as a non-collision event, it is possible that the accident involved a collision as indicated in Table 8-7. For these cases, it is important to identify the other vehicle involved. The main accident file constructed from TIFA includes a variable ('other vehicle type', see Section 7.1) that was generated for this purpose. Table 8-8 indicates the number of fatal involvements in which either no other vehicle was involved or a truck, car or tanker was involved by MHE given that a collision occurred (impact direction equal to front/rear or side). Table 8-9 provides the percentage of fatal accidents in which a collision occurred for the indicated MHE that either do not involve another vehicle or involve a truck, car or tanker. Note that for those cases in which no other vehicle is involved, the collision could have involved a hard object, soft object, non-fixed object or a train.

A comparison with non-fatal accidents is not available since a description of the other vehicle involved comparable to that developed from the TIFA file was not available for the GES file. 
Table 8-7 Overall Frequency of Impact Direction by MHE for Different Accident Thresholds

\begin{tabular}{|c|c|c|c|c|c|}
\hline MHE & \begin{tabular}{|l} 
Impact \\
Direction
\end{tabular} & GES-All & $\begin{array}{c}\text { GES-Fatal } \\
\text { or Tow-away }\end{array}$ & TIFA & GES-Fatal \\
\hline \multirow{4}{*}{$\begin{array}{l}\text { Truck } \\
\text { (including Tanker) }\end{array}$} & Front & $30.05 \%$ & $42.76 \%$ & $54.28 \%$ & $26.39 \%$ \\
\hline & Side & $46.95 \%$ & $24.10 \%$ & $18.02 \%$ & $33.63 \%$ \\
\hline & Rear & $22.95 \%$ & $33.04 \%$ & $27.71 \%$ & $36.90 \%$ \\
\hline & Non-collision & $0.04 \%$ & $0.09 \%$ & $0.00 \%$ & $3.07 \%$ \\
\hline \multirow[t]{4}{*}{$\overline{\mathrm{Car}}$} & Front & $29.86 \%$ & $30.77 \%$ & $59.60 \%$ & $54.26 \%$ \\
\hline & Side & $54.08 \%$ & $51.31 \%$ & $21.68 \%$ & $30.84 \%$ \\
\hline & Rear & $15.63 \%$ & $17.27 \%$ & $18.72 \%$ & $13.82 \%$ \\
\hline & Non-collision & $0.42 \%$ & $0.66 \%$ & $0.00 \%$ & $1.08 \%$ \\
\hline \multirow[t]{4}{*}{ Hard object } & Front & $28.12 \%$ & $43.49 \%$ & $83.63 \%$ & $100.00 \%$ \\
\hline & Side & $67.55 \%$ & $50.42 \%$ & $15.49 \%$ & $0.00 \%$ \\
\hline & Rear & $2.41 \%$ & $1.53 \%$ & $0.88 \%$ & $0.00 \%$ \\
\hline & Non-collision & $1.92 \%$ & $4.57 \%$ & $0.00 \%$ & $0.00 \%$ \\
\hline \multirow[t]{4}{*}{ Soft object } & $\mid \overline{\text { Front }}$ & $16.78 \%$ & $48.53 \%$ & $79.63 \%$ & $20.23 \%$ \\
\hline & \begin{tabular}{|l} 
Side \\
\end{tabular} & $76.44 \%$ & $44.05 \%$ & $18.52 \%$ & $76.24 \%$ \\
\hline & Rear & $4.11 \%$ & $1.17 \%$ & $1.85 \%$ & $3.53 \%$ \\
\hline & Non-collision & $2.67 \%$ & $6.24 \%$ & $0.00 \%$ & $0.00 \%$ \\
\hline \multirow[t]{4}{*}{ Non-fixed object } & Front & $46.30 \%$ & $71.52 \%$ & $63.52 \%$ & $88.30 \%$ \\
\hline & Side & $42.71 \%$ & $26.00 \%$ & $29.24 \%$ & $11.70 \%$ \\
\hline & Rear & $9.25 \%$ & $0.29 \%$ & $7.06 \%$ & $0.00 \%$ \\
\hline & Non-collision & $1.74 \%$ & $2.19 \%$ & $0.18 \%$ & $0.00 \%$ \\
\hline \multirow[t]{4}{*}{ Train } & Front & $19.34 \%$ & $25.10 \%$ & $49.28 \%$ & $0.00 \%$ \\
\hline & Side & $80.66 \%$ & $74.90 \%$ & $47.83 \%$ & $100.00 \%$ \\
\hline & Rear & $0.00 \%$ & $0.00 \%$ & $2.90 \%$ & $0.00 \%$ \\
\hline & Non-collision & $0.00 \%$ & $0.00 \%$ & $0.00 \%$ & $0.00 \%$ \\
\hline \multirow[t]{4}{*}{ Rollover } & Front & $5.81 \%$ & $6.33 \%$ & $39.08 \%$ & $28.89 \%$ \\
\hline & Side & $6.17 \%$ & $6.14 \%$ & $24.71 \%$ & $0.00 \%$ \\
\hline & Rear & $0.17 \%$ & $0.18 \%$ & $2.75 \%$ & $0.00 \%$ \\
\hline & Non-collision & $87.85 \%$ & $87.35 \%$ & $33.46 \%$ & $71.11 \%$ \\
\hline \multirow[t]{4}{*}{ Fire } & Front & $5.40 \%$ & $7.83 \%$ & $72.93 \%$ & $100.00 \%$ \\
\hline & Side & $5.29 \%$ & $7.67 \%$ & $12.15 \%$ & $0.00 \%$ \\
\hline & Rear & $0.00 \%$ & $0.00 \%$ & $6.63 \%$ & $0.00 \%$ \\
\hline & \begin{tabular}{|l} 
Non-collision \\
\end{tabular} & $89.32 \%$ & $84.51 \%$ & $8.29 \%$ & $0.00 \%$ \\
\hline \multirow{4}{*}{$\begin{array}{l}\text { Other } \\
\text { non-collision }\end{array}$} & Front & $0.59 \%$ & $1.64 \%$ & $25.42 \%$ & $0.00 \%$ \\
\hline & Side & $2.48 \%$ & $2.18 \%$ & $11.86 \%$ & $0.00 \%$ \\
\hline & Rear & $0.17 \%$ & $0.49 \%$ & $5.08 \%$ & $0.00 \%$ \\
\hline & Non-collision & $96.75 \%$ & $95.69 \%$ & $57.63 \%$ & $100.00 \%$ \\
\hline
\end{tabular}


Table 8-8 Weighted Number of Fatal Accidents in Which a Collision Occurred by MHE and Other Vehicle Involved

\begin{tabular}{|l|r|r|r|c|}
\hline \multirow{2}{*}{ MHE } & \multicolumn{4}{|c|}{ Other Vehicle Involved } \\
\cline { 2 - 5 } & None & Truck & Car & Tanker \\
\hline Truck & 0 & 876 & 0 & 0 \\
\hline Car & 0 & 0 & 9846 & 0 \\
\hline Tanker & 0 & 0 & 0 & 20 \\
\hline Hard Object & 156 & 12 & 60 & 0 \\
\hline Soft Object & 145 & 10 & 70 & 0 \\
\hline Non-fixed Object & 975 & 36 & 124 & 0 \\
\hline Train & 69 & 1 & 2 & 0 \\
\hline Rollover & 561 & 25 & 194 & 0 \\
\hline Fire & 80 & 34 & 74 & 0 \\
\hline Immersion & 12 & 1 & 3 & 0 \\
\hline
\end{tabular}

Table 8-9 Frequency of Other Vehicle Involved by MHE (Given a Collision)

\begin{tabular}{|l|r|r|r|r|}
\hline \multirow{2}{*}{ MHE } & \multicolumn{4}{|c|}{ Other Vehicle Involved } \\
\cline { 2 - 5 } & \multicolumn{1}{|c|}{ None } & Truck & \multicolumn{1}{c|}{ Car } & Tanker \\
\hline Truck & $0.00 \%$ & $100.00 \%$ & $0.00 \%$ & $0.00 \%$ \\
\hline Car & $0.00 \%$ & $0.00 \%$ & $100.00 \%$ & $0.00 \%$ \\
\hline Tanker & $0.00 \%$ & $0.00 \%$ & $0.00 \%$ & $100.00 \%$ \\
\hline Hard Object & $68.42 \%$ & $5.26 \%$ & $26.32 \%$ & $0.00 \%$ \\
\hline Soft Object & $64.44 \%$ & $4.44 \%$ & $31.11 \%$ & $0.00 \%$ \\
\hline Non-fixed Object & $85.90 \%$ & $3.17 \%$ & $10.93 \%$ & $0.00 \%$ \\
\hline Train & $95.83 \%$ & $1.39 \%$ & $2.78 \%$ & $0.00 \%$ \\
\hline Rollover & $71.92 \%$ & $3.21 \%$ & $24.87 \%$ & $0.00 \%$ \\
\hline Fire & $42.55 \%$ & $18.09 \%$ & $39.36 \%$ & $0.00 \%$ \\
\hline Immersion & $75.00 \%$ & $6.25 \%$ & $18.75 \%$ & $0.00 \%$ \\
\hline
\end{tabular}

\subsection{Impact Location}

Given a collision, the impact location defines which transport element on the case vehicle was impacted directly by the other vehicle or object: either (1) the tractor only or (2) the trailer and/or the tractor. In the DPTRA study, this information is used to determine the threat to the integrity of the SST. The trailer is likely to have greater damage if it is directly impacted in the collision. If the tractor is impacted first, collision energy is absorbed by crush and deformation of the tractor, which reduces the energy imparted to the trailer. 
The supplemental collision severity data were used to determine impact location. The weighted number of fatal accidents by MHE, impact direction and impact location are listed in Table 8-10; impact location frequencies for fatal accidents with a given MHE and impact direction are indicated in Table 8-11. The procedures used to derive the impact location frequencies are based on the following observations and assumptions:

Table 8-10 Weighted Number of Fatal Accidents by Impact Location, Direction and MHE

\begin{tabular}{|c|c|c|c|c|c|c|}
\hline \multirow[b]{2}{*}{ MHE } & \multirow{2}{*}{$\begin{array}{l}\text { Impact } \\
\text { Direction }\end{array}$} & \multicolumn{5}{|c|}{ Impact Location } \\
\hline & & Tractor Only & Trailer & Subtotal & Unknown & Total \\
\hline \multirow[t]{4}{*}{ Truck/Tanker } & Front & 206 & 32 & 238 & 238 & 476 \\
\hline & Side & 36 & 46 & 82 & 76 & 158 \\
\hline & Rear & 4 & 117 & 121 & 122 & 243 \\
\hline & Other & 2 & 0 & 2 & 17 & 19 \\
\hline \multirow[t]{4}{*}{ Car } & Front & 270 & 3 & 273 & 5545 & 5818 \\
\hline & Side & 34 & 66 & 100 & 2016 & 2116 \\
\hline & Rear & 2 & 93 & 95 & 1732 & 1827 \\
\hline & Other & 2 & 0 & 2 & 83 & 85 \\
\hline \multirow[t]{4}{*}{ Objects } & Front & 323 & 12 & 335 & 719 & 1054 \\
\hline & Side & 61 & 19 & 80 & 314 & 394 \\
\hline & Rear & 3 & 2 & 5 & 78 & 83 \\
\hline & Other & 7 & 4 & 11 & 46 & 57 \\
\hline \multirow[t]{4}{*}{ Train } & Front & 25 & 0 & 25 & 9 & 34 \\
\hline & Side & 20 & 8 & 28 & 5 & 33 \\
\hline & Rear & 0 & 2 & 2 & 0 & 2 \\
\hline & Other & 0 & 3 & 3 & 0 & 3 \\
\hline \multirow{4}{*}{$\begin{array}{l}\text { Rollover/ } \\
\text { Immersion }\end{array}$} & Front & 142 & 7 & 149 & 159 & 308 \\
\hline & Side & 51 & 22 & 73 & 119 & 192 \\
\hline & Rear & 1 & 5 & 6 & 15 & 21 \\
\hline & Other & 13 & 8 & 21 & 254 & 275 \\
\hline \multirow[t]{4}{*}{ Fire } & Front & 75 & 2 & 77 & 77 & 154 \\
\hline & Side & 12 & 5 & 17 & 12 & 29 \\
\hline & Rear & 1 & 8 & 9 & 6 & 15 \\
\hline & Other & 8 & 3 & 11 & 27 & 38 \\
\hline \multirow[t]{4}{*}{ Unknown } & Front & 7 & 0 & 7 & 13 & 20 \\
\hline & Side & 0 & 3 & 3 & 9 & 12 \\
\hline & Rear & 0 & 2 & 2 & 5 & 7 \\
\hline & Other & 6 & 0 & 6 & 43 & 49 \\
\hline Total & & 1311 & 472 & 1783 & 11739 & 13522 \\
\hline
\end{tabular}


Table 8-11 Frequency of Impact Location by Impact Direction and MHE (Fatal Accidents)

\begin{tabular}{|l|c|r|r|}
\hline \multirow{3}{*}{ MHE } & Impact & \multicolumn{2}{|c|}{ Impact Location } \\
\cline { 3 - 4 } & Direction & Tractor Only & Trailer \\
\hline \multirow{3}{*}{ Car } & Front & $86.55 \%$ & $13.45 \%$ \\
\cline { 2 - 4 } & Side & $43.90 \%$ & $56.10 \%$ \\
\cline { 2 - 4 } & Rear & $3.31 \%$ & $96.69 \%$ \\
\hline Objects & Front & $98.90 \%$ & $1.10 \%$ \\
\cline { 2 - 4 } & Side & $34.00 \%$ & $66.00 \%$ \\
\cline { 2 - 4 } & Rear & $2.11 \%$ & $97.89 \%$ \\
\hline \multirow{3}{*}{ Train } & Front & $96.42 \%$ & $3.58 \%$ \\
\cline { 2 - 4 } & Side & $76.25 \%$ & $23.75 \%$ \\
\cline { 2 - 4 } & Rear & $60.00 \%$ & $40.00 \%$ \\
\hline Rollover/Immersion & Front & $100.00 \%$ & $0.00 \%$ \\
\cline { 2 - 4 } & Side & $71.43 \%$ & $28.57 \%$ \\
\cline { 2 - 4 } & Rear & $0.00 \%$ & $100.00 \%$ \\
\cline { 2 - 4 } & Front & $95.30 \%$ & $4.70 \%$ \\
\cline { 2 - 4 } & Side & $69.86 \%$ & $30.14 \%$ \\
\cline { 2 - 4 } & Rear & $16.67 \%$ & $83.33 \%$ \\
\hline Fire & Front & $97.40 \%$ & $2.60 \%$ \\
\cline { 2 - 4 } & Side & $70.59 \%$ & $29.41 \%$ \\
\cline { 2 - 4 } & Rear & $11.11 \%$ & $88.89 \%$ \\
\hline
\end{tabular}

1. The data for impact location for truck and tanker collisions were combined so that the estimated impact location frequencies are the same for accidents in which the MHE is coded as collision with a heavy truck or involvement with a tanker. ${ }^{12}$

2. Data for impact location for collisions with the three types of objects considered (fixed, hard objects; fixed, soft objects and non-fixed objects) were also combined.

A comparison with non-fatal accidents is not available since a description of the impact location comparable to that developed using supplementary data for the TIFA file was not available for the GES file. A comparison made with Michigan data in Reference 1

\footnotetext{
${ }^{12}$ Many of the characteristics of accidents involving heavy trucks and those involving tankers should be similar. The primary reason for differentiating these two types of vehicles in the event tree is that the amount of fuel carried in a tanker represents a much greater fire threat than that posed by an ordinary heavy truck. However, it is assumed that the characteristics of a collision event (i.e., distributions of $\mathrm{IL}$ and peak contact velocity) involving another heavy truck and those of collision event involving a tanker would be similar.
} 
demonstrated that, with respect to impact location, the TIFA accident data represent a class of accidents that are in general more threatening to the cargo in a semi-trailer (because side impacts into the trailer are more threatening than side impacts into the tractor) than tow-away accidents in general.

\subsection{Rollover Occurrence}

Accidents may involve a rollover even if the MHE is not coded as rollover. The main accident file constructed from TIFA includes a variable that indicates if the case vehicle experienced a rollover. This variable is used directly to determine rollover occurrence. The weighted number of fatal involvements in which the case vehicle experienced a rollover are shown in Table 8-12 by MHE and impact direction. The rollover occurrence frequencies for a given MHE and impact direction are listed in Table 8-13. These frequencies were calculated as the ratio of the counts listed in Table 8-12 to the count in the corresponding cell of Table 8-5. For six of the ten MHEs, impact direction equal to non-collision is precluded because the event is a collision by definition. These cases are indicated by na (not applicable) in the column listing the frequency of rollovers given a non-collision.

Table 8-12 Weighted Number of Fatal Accidents with Rollover by MHE and ID

\begin{tabular}{|c|c|c|c|c|c|c|}
\hline \multirow[b]{2}{*}{ MHE } & \multicolumn{5}{|c|}{ Impact Direction } & \multirow[b]{2}{*}{ Total } \\
\hline & Front & Side & Rear & Non-collision & Unknown & \\
\hline Truck & 34 & 22 & 6 & 0 & 0 & 62 \\
\hline Car & 303 & 131 & 22 & 0 & 9 & 465 \\
\hline Tanker & 0 & 0 & 1 & 0 & 0 & 1 \\
\hline Hard Object & 46 & 17 & 0 & 0 & 1 & 64 \\
\hline Soft Object & 45 & 20 & $\overline{0}$ & 0 & 2 & 67 \\
\hline Non-Fixed & 26 & 8 & 2 & 0 & 0 & 36 \\
\hline Train & 4 & 2 & 0 & 0 & 0 & 6 \\
\hline Rollover & 299 & 189 & 21 & 256 & 15 & 780 \\
\hline Fire & 38 & 7 & 4 & 14 & 6 & 69 \\
\hline Immersion & 4 & 0 & $\overline{0}$ & 2 & 2 & 8 \\
\hline Unknown & 19 & 7 & 2 & 12 & 7 & 47 \\
\hline Total & 818 & 403 & 58 & 284 & 42 & 1605 \\
\hline
\end{tabular}


Table 8-13 Frequency of Rollover by MHE and Impact Direction (Fatal Accidents)

\begin{tabular}{|l|r|r|r|c|}
\hline MHE & \multicolumn{1}{|c|}{ Front } & \multicolumn{1}{c|}{ Side } & Rear & Non-collision \\
\hline Truck & $7.26 \%$ & $14.67 \%$ & $2.51 \%$ & na \\
\hline Car & $5.21 \%$ & $6.19 \%$ & $1.20 \%$ & na \\
\hline Tanker & $0.00 \%$ & $0.00 \%$ & $25.00 \%$ & na \\
\hline Hard Object & $24.34 \%$ & $48.57 \%$ & $0.00 \%$ & na \\
\hline Soft Object & $26.16 \%$ & $50.00 \%$ & $0.00 \%$ & na \\
\hline Non-Fixed & $3.75 \%$ & $2.51 \%$ & $2.60 \%$ & $0.00 \%$ \\
\hline Train & $11.76 \%$ & $6.06 \%$ & $0.00 \%$ & na \\
\hline Rollover & $100.00 \%$ & $100.00 \%$ & $100.00 \%$ & $100.00 \%$ \\
\hline Fire & $28.79 \%$ & $31.82 \%$ & $33.33 \%$ & $93.33 \%$ \\
\hline Immersion & $44.44 \%$ & $0.00 \%$ & na & $100.00 \%$ \\
\hline
\end{tabular}

Table 8-14 lists the overall frequencies of rollover occurrence by MHE based on TIFA data and GES data for different accident severity levels (i.e., all, fatal or tow-away, and fatal). The estimates obtained with GES are not always reliable since the sample sizes are small or even non-existent (as indicated by an asterisk in Table 8-14) in many of the cells of this two-way distribution, particularly for fatal accidents. TIFA provides a much larger sample size, such that all cells in this two-way distribution are reasonably well-populated. Furthermore, the frequency of rollover occurrence for fatal accidents as obtained from the TIFA data is considerably greater than that for tow-away/fatal accidents as obtained from GES. This is consistent with the notion that fatal accidents generally impose a more severe environment on the cargo than do tow-away accidents.

Table 8-14 Overall Frequency of Rollover by MHE and Accident Reporting Threshold

\begin{tabular}{|l|r|r|r|r|}
\hline & GES-All & $\begin{array}{c}\text { GES-Fatal } \\
\text { or Tow-away }\end{array}$ & TIFA & GES-Fatal \\
\hline Truck (including tanker) & $0.04 \%$ & $0.10 \%$ & $7.03 \%$ & $0.00 \%$ \\
\hline Car & $0.14 \%$ & $0.37 \%$ & $4.72 \%$ & $0.92 \%$ \\
\hline Hard Object & $0.99 \%$ & $2.41 \%$ & $28.07 \%$ & $0.00 \%$ \\
\hline Soft Object & $0.37 \%$ & $2.33 \%$ & $29.78 \%$ & $14.05 \%$ \\
\hline Non-Fixed Object & $0.04 \%$ & $0.38 \%$ & $3.17 \%$ & $0.00 \%$ \\
\hline Train & $3.34 \%$ & $4.25 \%$ & $8.33 \%$ & $0.00 \%$ \\
\hline Rollover & $100.00 \%$ & $100.00 \%$ & $100.00 \%$ & $100.00 \%$ \\
\hline Fire & $0.00 \%$ & $0.00 \%$ & $36.70 \%$ & $0.00 \%$ \\
\hline Immersion & $0.00 \%$ & $0.00 \%$ & $0.00 \%$ & $*$ \\
\hline
\end{tabular}

*No cases with the indicated description exist in the 1992-96 GES file. 


\subsection{Fire Occurrence}

Accidents may involve a fire even if the MHE is not coded as fire. The main accident file constructed from TIFA includes a variable that indicates if any vehicle involved in the accident experienced a fire. This variable is used directly to determine fire occurrence. The weighted number of fatal involvements in which a fire occurred are shown in Table 8-15 by MHE and impact direction. The fire occurrence frequencies for a given MHE and impact direction are listed in Table 8-16. These frequencies were calculated as the ratio of the counts listed in Table 8-15 to the count in the corresponding cell of Table 8-5. For six of the ten MHEs, impact direction equal to non-collision is precluded. These cases are indicated by na (not applicable) in the column listing the frequency of fire given a non-collision. Although fire would seem to be precluded when the MHE is immersion, this is not the case. Many of the cases in which the MHE is immersion involved ejection of a vehicle occupant into water; the vehicle itself is not immersed and fire is possible.

Table 8-15 Weighted Number of Fatal Accidents with Fire by Impact Direction and MHE

\begin{tabular}{|c|c|c|c|c|c|c|}
\hline \multirow[b]{2}{*}{ MHE } & \multicolumn{5}{|c|}{ Impact Direction } & \multirow[b]{2}{*}{ Total } \\
\hline & Front & Side & Rear & Non-collision & Unknown & \\
\hline Truck & 62 & 16 & 50 & 0 & 0 & 128 \\
\hline $\mathrm{Car}$ & 202 & 48 & 15 & 0 & 3 & 268 \\
\hline Tanker & 1 & 3 & 0 & 0 & 0 & 4 \\
\hline Hard Object & 19 & 0 & 1 & 0 & 0 & 20 \\
\hline Soft Object & 11 & 5 & 0 & 0 & 1 & 17 \\
\hline Non-Fixed & 7 & 6 & 3 & 0 & 0 & 16 \\
\hline Train & 8 & 6 & 1 & 0 & 0 & 15 \\
\hline Rollover & 15 & 8 & 1 & 12 & 2 & 38 \\
\hline Fire & 132 & 22 & 12 & 15 & 7 & 188 \\
\hline Immersion & 1 & 0 & 0 & 0 & 0 & 1 \\
\hline Unknown & 24 & 9 & 3 & 11 & 5 & 52 \\
\hline Total & 482 & 123 & 86 & 38 & 18 & 747 \\
\hline
\end{tabular}


Table 8-16 Frequency of Fire by MHE and Impact Direction (Fatal Accidents)

\begin{tabular}{|l|r|r|r|c|}
\hline MHE & \multicolumn{1}{|l|}{ Front } & \multicolumn{1}{l|}{ Side } & \multicolumn{1}{l|}{ Rear } & Non-collision \\
\hline Truck & $13.25 \%$ & $10.67 \%$ & $20.92 \%$ & na \\
\hline Car & $3.47 \%$ & $2.27 \%$ & $0.82 \%$ & na \\
\hline Tanker & $12.50 \%$ & $37.50 \%$ & $0.00 \%$ & na \\
\hline Hard Object & $10.05 \%$ & $0.00 \%$ & $50.00 \%$ & na \\
\hline Soft Object & $6.40 \%$ & $12.50 \%$ & $0.00 \%$ & na \\
\hline Non-Fixed & $1.01 \%$ & $1.88 \%$ & $3.90 \%$ & na \\
\hline Train & $23.53 \%$ & $18.18 \%$ & $50.00 \%$ & na \\
\hline Rollover & $5.02 \%$ & $4.23 \%$ & $4.76 \%$ & $4.69 \%$ \\
\hline Fire & $100.00 \%$ & $100.00 \%$ & $100.00 \%$ & $100.00 \%$ \\
\hline Immersion & $11.11 \%$ & $0.00 \%$ & na & $0.00 \%$ \\
\hline
\end{tabular}

Table 8-17 lists the overall frequencies of fire occurrence by MHE based on TIFA data and GES data for different accident severity levels (i.e., all, fatal or tow-away, and fatal). The estimates obtained with GES are not reliable since the sample sizes are small or even nonexistent (as indicated by an asterisk in Table 8-17) in many of the cells of this two-way distribution, particularly for fatal accidents. TIFA provides a much larger sample size, such that all cells in this two-way distribution are reasonably well-populated. Furthermore, the frequencies of fire occurrence for fatal accidents as obtained from the TIFA data are considerably greater than those for lower accident reporting thresholds as obtained from GES. This is consistent with the notion that fatal accidents generally impose a more severe environment on the cargo than do tow-away accidents.

Table 8-17 Overall Frequency of Fire by MHE for Different Accident Reporting Thresholds

\begin{tabular}{|l|r|r|r|r|}
\hline DPTRA tree & GES-All & $\begin{array}{c}\text { GES-Fatal } \\
\text { or Tow-away }\end{array}$ & \multicolumn{1}{|c|}{ TIFA } & GES-Fatal \\
\hline Truck (Including Tanker) & $0.44 \%$ & $0.89 \%$ & $14.73 \%$ & $0.00 \%$ \\
\hline Car & $0.06 \%$ & $0.08 \%$ & $2.72 \%$ & $0.00 \%$ \\
\hline Hard Object & $0.09 \%$ & $0.21 \%$ & $8.77 \%$ & $0.00 \%$ \\
\hline Soft Object & $0.00 \%$ & $0.00 \%$ & $7.56 \%$ & $0.00 \%$ \\
\hline Non-Fixed & $0.03 \%$ & $0.24 \%$ & $1.41 \%$ & $0.00 \%$ \\
\hline Train & $0.00 \%$ & $0.00 \%$ & $20.83 \%$ & $0.00 \%$ \\
\hline Rollover & $0.52 \%$ & $0.57 \%$ & $4.87 \%$ & $0.00 \%$ \\
\hline Fire & $100.00 \%$ & $100.00 \%$ & $100.00 \%$ & $100.00 \%$ \\
\hline Immersion & $0.00 \%$ & $0.00 \%$ & $6.25 \%$ & $*$ \\
\hline
\end{tabular}

*No cases with the indicated description exist in the 1992-96 GES file. 


\section{Discussion and Analysis of Accident Severities}

This chapter addresses the severity of collisions, rollovers and fires in transportation accidents. Each of these events may occur separately or in any combination with the other two. The frequencies with which these events occur (separately and in combination) are defined by the factors used to characterize the accident, as described in Chapter 8.

\subsection{Collision}

. Three factors are used to assess collision severity in the DPTRA study: (1) peak contact velocity, which is used to evaluate cargo damage from impact, puncture, and crush, (2) collision energy absorbed by the AT/SST, which is used to determine the extent of damage to the trailer, and (3) the maximum deceleration of the trailer, which is used to evaluate the response of the tie-down system. The collision energy and maximum deceleration depend on the peak contact velocity, as described later in this section. Thus, the collision severity depends only on the type of accident (as characterized by the six factors described in Chapter 8) and the peak contact velocity.

\subsubsection{Peak Contact Velocity}

The primary metric used to characterize collision severity is the peak contact velocity, which is a measure of the maximum relative velocity (ignoring effects of the tie-down system or other restraint systems) with which the cargo can strike the interior trailer walls. A more detailed discussion of peak contact velocity can be found in Reference 14 . The peak contact velocity for a case vehicle involved in a collision is equal to its initial velocity minus the final common velocity, which, based on conservation of momentum, results in the following expression:

$$
P C V=\frac{V_{r}}{1+M / m}
$$

where $V_{r}$ is the relative velocity between the case vehicle and the other vehicle or object involved, $M$ is the effective mass of the case vehicle and $m$ is the effective mass of the other vehicle or object involved.

Reference 1 compared the distributions of peak contact velocity for fatal (as obtained from the supplemental TIFA data) and non-fatal tow-away (as obtained from Michigan data) accidents (1) in which the most harmful event was coded as a collision with another medium or heavy truck and (2) in which the most harmful event was a collision with a fixed object. The likelihood of a peak contact velocity greater than about $10 \mathrm{mph}$ was consistently greater for fatal accidents than it is for non-fatal, tow-away accidents (i.e., the collision severity of fatal accidents is in general more threatening than that of non-fatal tow-away accidents). 
For a specific type of vehicle, such as the AT/SST, the best estimate of the distributions of peak contact velocity can be determined by substituting the effective mass of the vehicle of interest for the effective mass of the case vehicle (this is appropriate since the case vehicle is being used as a surrogate for the vehicle of interest). Section 6.1 provides a discussion of how the effective mass of the case vehicle was determined. In essence, the effective mass is determined by identifying the elements of the case vehicle that participate in momentum exchange during the collision. For front/rear (head-on and rear-end) accidents, the effective mass is normally equal to the GCW. For side accidents, it is possible that only the tractor or only the trailer may participate in the momentum exchange depending on the impact location. The effective mass of an empty AT/SST for a particular case was determined according to which elements of the case vehicle participated in the momentum exchange, as outlined in Table 9-1. Figures 9-1 through 9-3 show the peak contact velocity distributions obtained by substituting the effective mass of an empty AT/SST for the effective mass of the case vehicle $^{13}$. The distributions are dependent on the MHE and impact direction.

Table 9-1 Computation of Effective Mass for the AT/SST ${ }^{14}$

\begin{tabular}{|c|c|c|}
\hline Mass Element & Code Value & Effective Mass of AT/SST(lb.) \\
\hline Tractor and Trailer & 1 & 64,500 \\
\hline Tractor only & 2 & 23,800 \\
\hline Trailer only & 3 & 40,700 \\
\hline
\end{tabular}

The distributions in Figures 9-1 through 9-3 were derived based on the following observations and assumptions:

1. The data for truck and tanker collisions were combined (because the number of records for tanker collisions in the supplemental TIFA data is small) so that the distribution of peak contact velocity is taken to be the same for accidents in which the MHE is coded as collision with a heavy truck or involvement with a tanker. Many of the characteristics of accidents involving heavy trucks and those involving tankers should be similar. The primary reason for differentiating these two types of vehicles in the event tree is that the amount of fuel carried in a tanker represents a much greater fire threat than that posed by an ordinary heavy truck. However, it is assumed that the characteristics of a collision event (i.e., distributions of impact location and peak contact velocity) involving another heavy truck and those of collision event involving a tanker are similar.

\footnotetext{
${ }^{13}$ In the risk assessment, the distributions of PCV are recomputed for each cargo considered based on the loaded weight of the trailer, using the same logic described here.

${ }^{14}$ The weights in this table are the minimum unloaded weights for all armored tractors and secure trailers in the DOE fleet.
} 
2. The data for collisions with cars and non-fixed objects were combined so that the distribution of peak contact velocity is taken to be the same for accidents in which the MHE is coded as collision with a car or light truck or collision with a non-fixed object. In general, these types of collisions are not very threatening to the contents of the SST or SGT, and so there is little to be gained by further differentiation.

3. The data for collisions with hard and soft fixed objects were combined so that the distribution of peak contact velocity is taken to be the same for accidents in which the MHE is coded as collision with a hard, fixed object or as a collision with a soft, fixed object. This combination is justified because the characteristics of a collision event (i.e., distributions of impact location and peak contact velocity) involving fixed objects should be similar.

4. The data for rollover and immersion were combined (because the number of records in the supplemental TIFA data is small) so that the distribution of peak contact velocity is taken to be the same for accidents in which the MHE is coded as rollover or immersion.

5. The upper bound for all the peak contact velocity distributions was assumed to be $5 \mathrm{mph}$ greater than the maximum value obtained from the data. This assumed upper bound is intended to reflect accidents that could occur (albeit rarely) but were not observed in 19921996 data, nor in the 1980-1990 data [1].

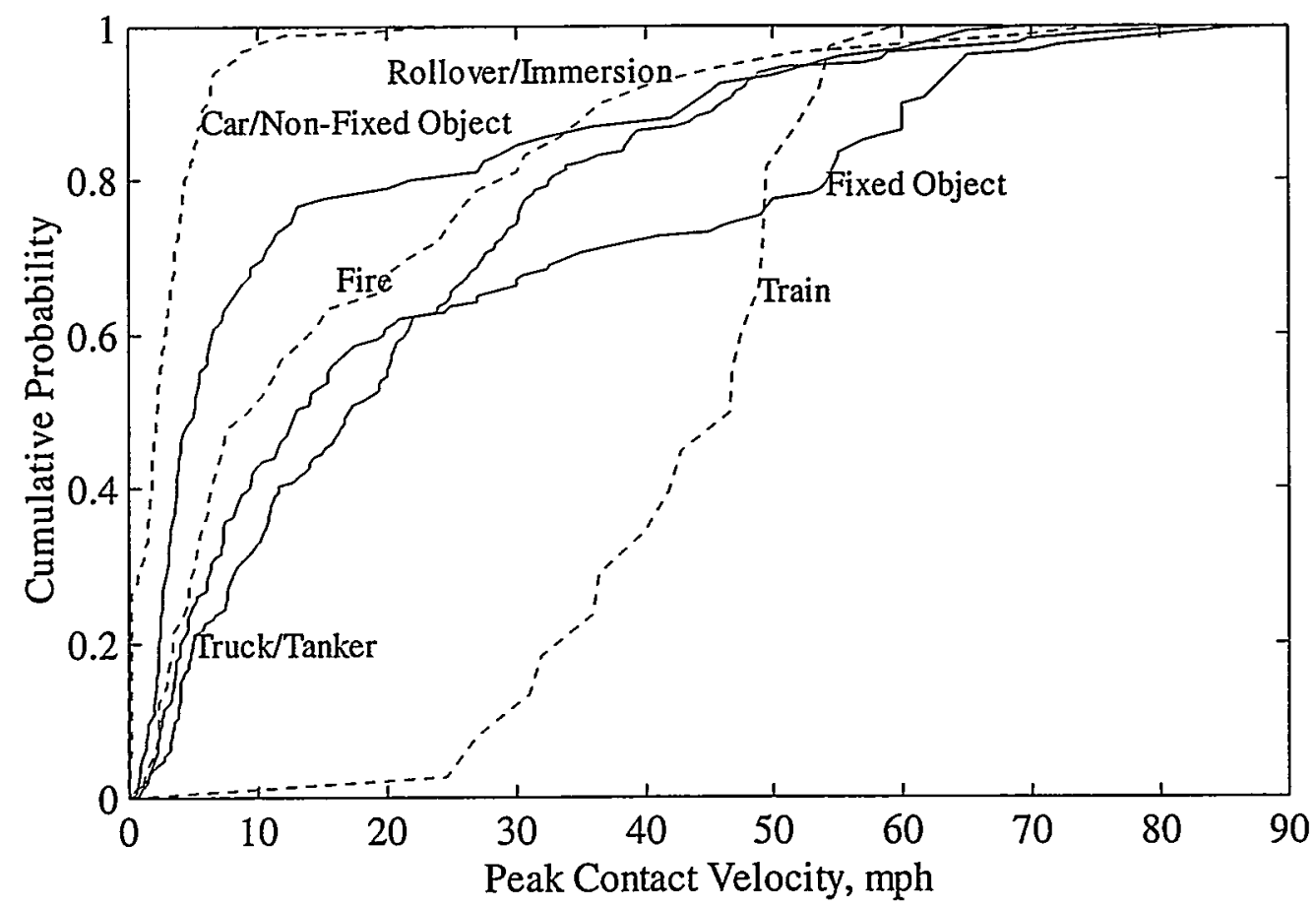

Figure 9-1 AT/SST Peak Contact Velocity Distributions by MHE, ID=Front 


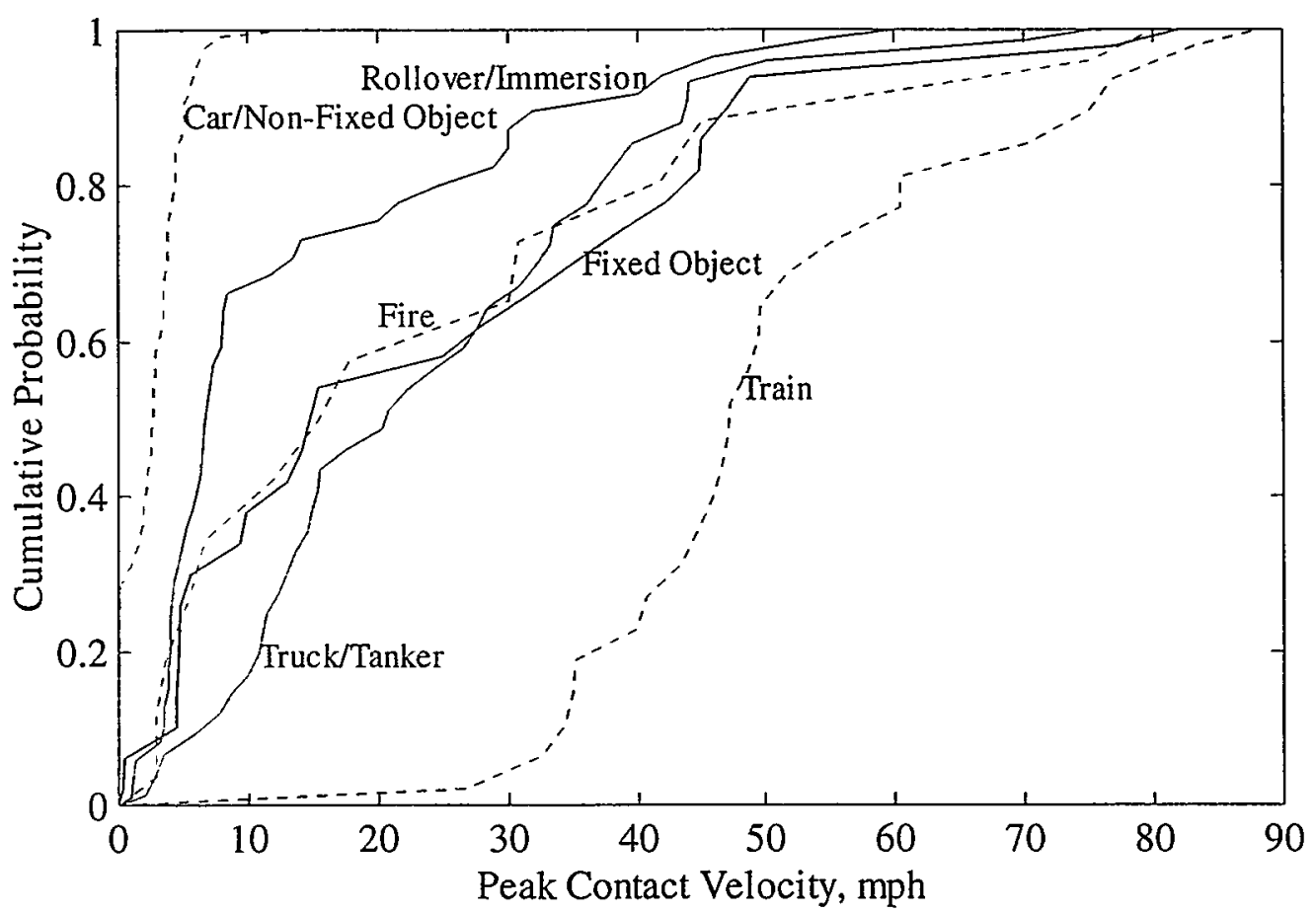

Figure 9-2 AT/SST Peak Contact Velocity Distributions by MHE, $\mathrm{D}=$ Side

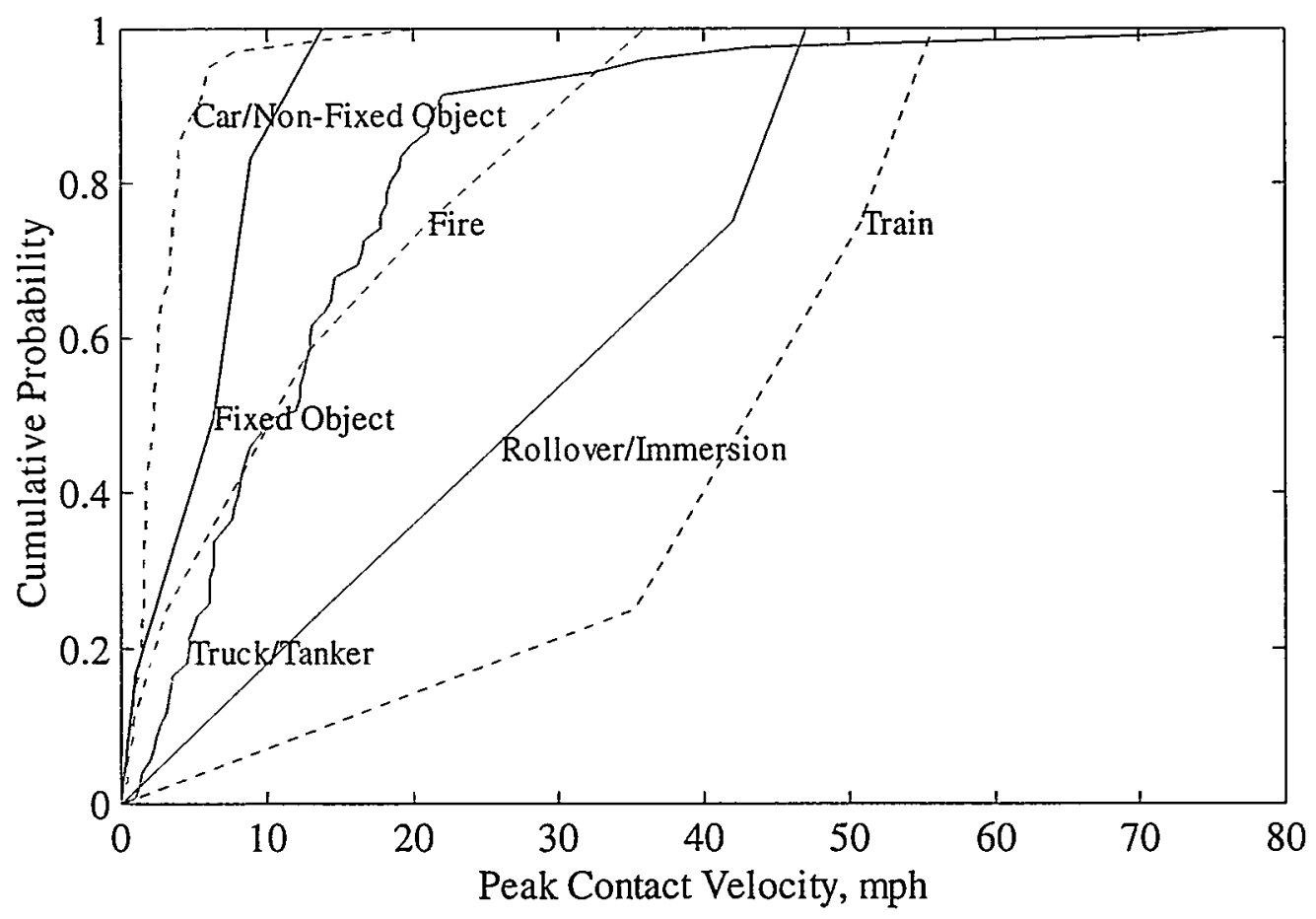

Figure 9-3 AT/SST Peak Contact Velocity Distributions by MHE, $\mathrm{D}=$ Rear 


\subsubsection{Collision Energy}

The energy absorbed in a collision is a measure of the work done, which is related to the deformation and damage of the vehicles. Based on conservation of momentum and conservation of energy laws and ignoring rebound, which is conservative, the total energy absorbed in a collision, $E_{a}$, can be expressed as:

$$
E_{a}=0.5\left\{M(P C V)^{2}+m(p c v)^{2}\right\}
$$

where $M, P C V$ and $m$ are defined as before and $p c v$ represent the peak contact velocity of the other vehicle or object involved in the collision, which is given by $\frac{V_{r}}{1+m / M}$. The fraction of total energy that is absorbed by each vehicle depends on crush characteristics of the vehicles and objects involved and the impact locations. It is assumed that the collision energy absorbed by the AT/SST is:

$$
E=0.5 M(P C V)^{2} \text {. }
$$

By substituting $p c v=\frac{M}{m} P C V$ into Eqn. 9-2 it can be shown that the fraction of the total collision energy absorbed by the AT/SST is:

$$
\frac{E}{E_{a}}=\frac{m}{M+m} \text {. }
$$

This provides a reasonable estimate if stiffness of a vehicle or object is proportional to its mass. The frame and body of the SST are considerably stronger and stiffer than most semitrailers with comparable GCWs, so this assumption is certainly conservative when applied to the AT/SST. The appropriateness of this assumption for other makes and models of tractor semi-trailers is not addressed here.

\subsubsection{Maximum Deceleration}

The response of the tie-down system or other restraint system depends on the maximum deceleration arising from the collision. The maximum deceleration is strongly dependent on details of the vehicle design, the characteristics of the other vehicle or object involved, the impact direction and the impact location. For the AT/SST, a simple, empirically derived relationship between the maximum deceleration, $a_{\max }$, and the peak contact velocity, $P C V$, was derived based on crash testing conducted on 1:4-scale models:

$$
a_{\max }=\frac{2 P C V}{t_{d}}
$$

where $\dot{t}_{d}$ is the collision duration (i.e., the time elapsed between initial impact and the point at which a final common velocity is reached). For a specific vehicle, the collision duration can 
be approximated as a constant given the type of the other vehicle or object involved, the impact direction and the impact location. Collision durations for the AT/SST were derived based on 1:4-scale model crash tests, but the discussion of those results is outside the scope of this report. The applicability of this approach to evaluating maximum deceleration to tractor semi-trailers other than the AT/SST is also not addressed in this report.

\subsection{Rollover}

For the AT/SST, the primary concern in a rollover event is the possibility of damage to the trailer walls. The maximum acceleration (or deceleration) generated in rollovers is less than that required to threaten the functionality of the tie-down system in the SST. As an approximation, damage to the walls of the SST is related to the skid distance. A distribution of skid distance can be estimated using the data on rollover events in Reference 1, as shown in Figure 9-4. A curve fit using an exponential function (i.e., a Weibull distribution) was developed that closely matches the data, i.e., $P(D)=1-e^{-(D / 120)^{1.3}}$.

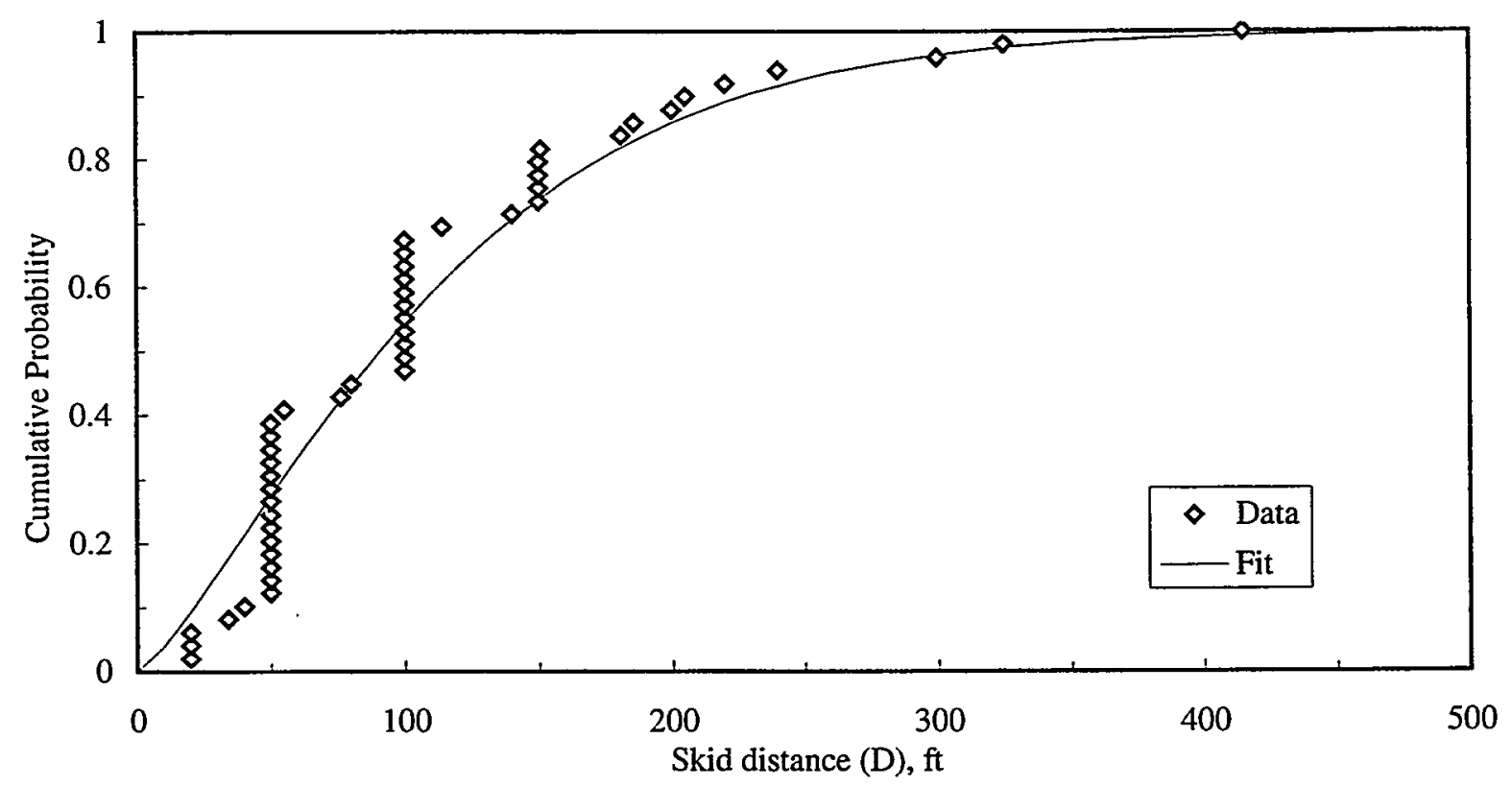

Figure 9-4 Distribution for Skid Distance Associated with Rollover

\subsection{Fire}

In the DPTRA study, the MELTER computer code [18] was used to analyze the thermal response of cargoes in an SST to fire environments associated with transportation accidents. The fire characteristics that are important in such a simulation include the effective fire temperature, separation between the fire and the trailer, size, and the fire duration. The fire data collection effort included information that can be used to evaluate these characteristics, directly and/or indirectly. As in the previous report [1], the distributions for fire size and fire duration were obtained from an analytical simulation, in which the parameters were chosen to 
provide reasonable agreement with the limited data available. The effective fire temperature is derived indirectly based on the likely fuel source of fires involved in transportation accidents. Of the four fire characteristics needed, only the fire separation is derived directly and exclusively from the data collected on fatal accidents.

Fires associated with fatal accidents are, in general, more severe than those associated with non-fatals [1]. Fires associated with fatal accidents are more likely to involve the fuel systems and/or cargo than those associated with non-fatal accidents. For fires associated with fatal accidents, the fuel of the involved vehicles is almost always involved. This suggests that there is a higher frequency of leakage and dispersion of fuel in fatal accidents, which is again consistent with the notion that collisions and resulting damage to vehicles associated with fatal accidents is more severe than non-fatal accidents. From a practical standpoint, this also suggests that it is not overly conservative to treat all fires associated with fatal accidents as if they were (hydrocarbon) fuel fires. This is in fact the key assumption adopted in developing the models used to develop distributions for effective fire temperature, size and duration.

\subsubsection{Effective Fire Temperature ${ }^{15}$}

The effective fire temperature, $T$, can be related to the emissive power of the fire, $E_{f}$, by the following expression:

$$
E_{f}=\sigma T^{4}
$$

where $\sigma$ is the Stefan-Boltzman constant, which is equal to $5.67 \times 10^{-8} \mathrm{~W} / \mathrm{m}^{2}-\mathrm{K}^{4}$. The effective fire temperature depends on the fuel source, location of the object being heated relative to the fire, atmospheric conditions and other factors. As discussed previously, the fuel source for all fires associated with fatal accidents is assumed to be a liquid or gas hydrocarbon. Two cases are considered with respect to the location of the object being heated: (1) engulfed (in the fire) and (2) out of the fire.

Figure 9-5 shows distributions of effective fire temperature obtained from References 4 and 19. Reference 19 actually provides probability estimates at just a few discrete temperatures; the curve fit was developed separately. ${ }^{16}$ These distributions are applicable when the object being heated is in the fire. In the DPTRA study, the curve fit to the data obtained from Reference 19 was used since the data in Reference 19 are based on actual measurements of temperatures for a number of different fuel fires. The variation in effective fire temperature is due to the type of fuel, atmoshperic conditions, and other factors.

\footnotetext{
${ }^{15}$ This section is taken directly from Reference 1 and is included here for continuity and completeness.

${ }^{16}$ Personal communication with M. K. Fuentes, May 4, 1994.
} 


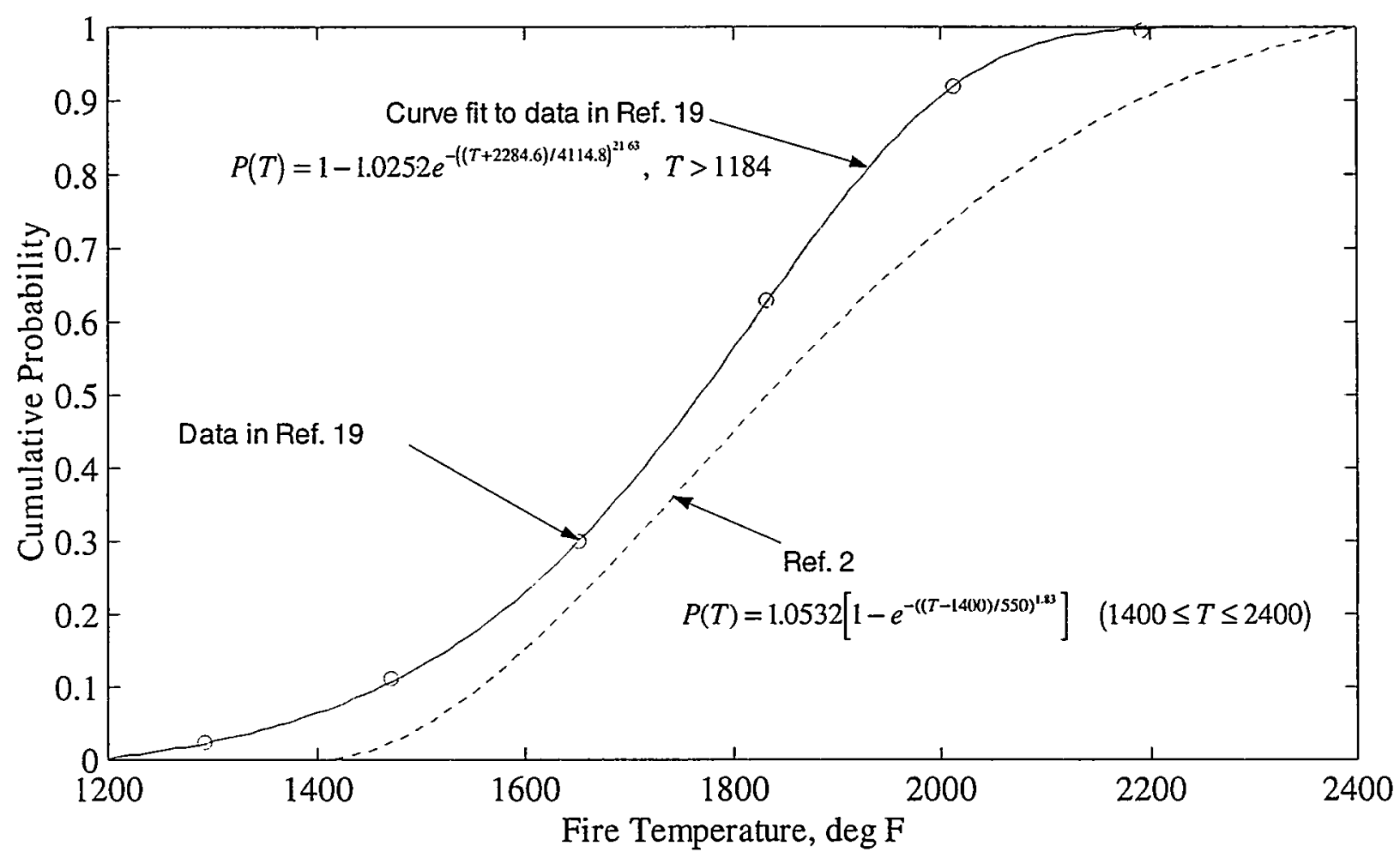

Figure 9-5 Distribution for Effective Fire Temperature--Object Heated is in the Fire

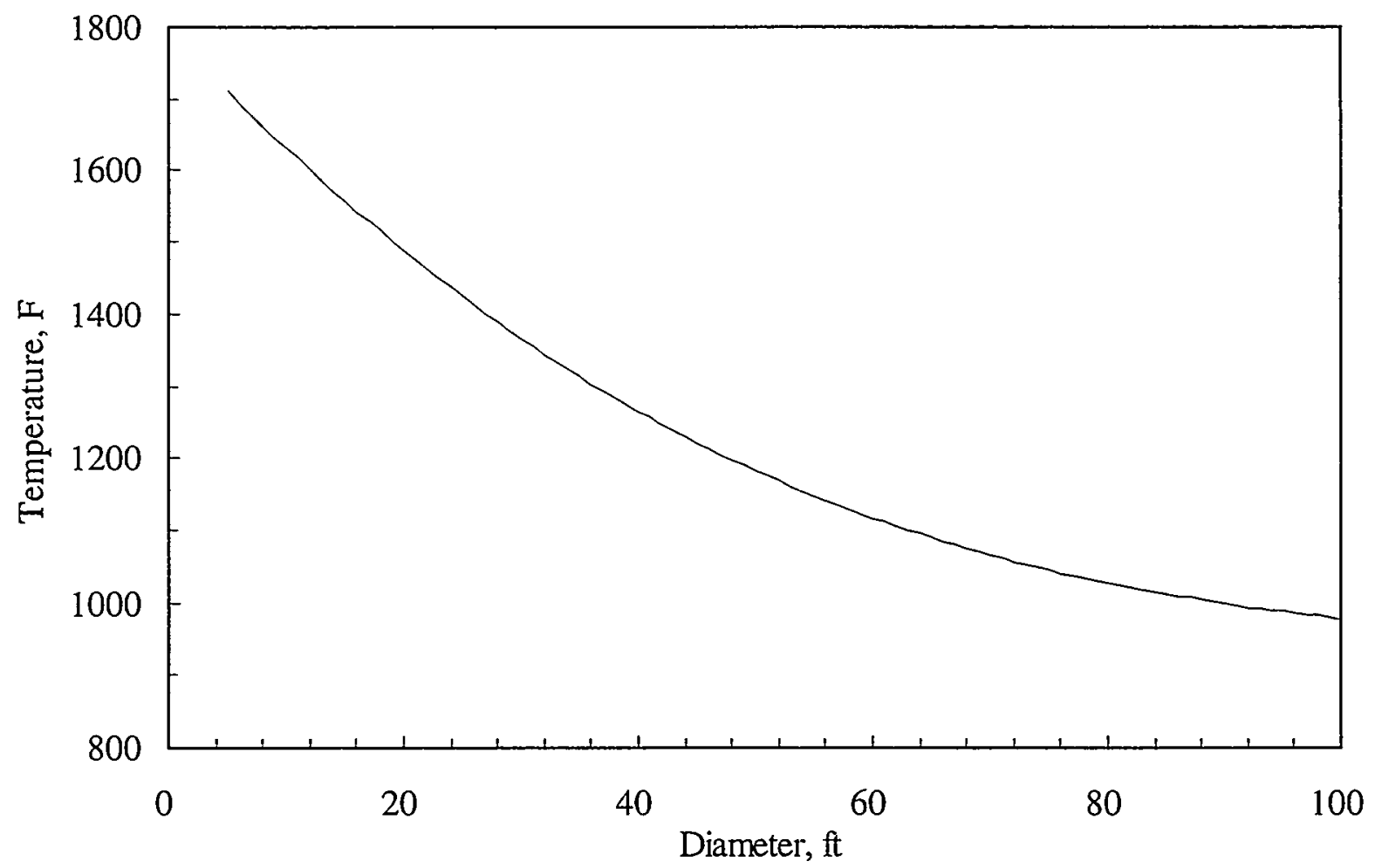

Figure 9-6 Effective Fire Temperature vs. Fire Diameter--Object Heated is out of the Fire 
When the object being heated is out of the fire, the emmisive power of the fire is reduced due to the effects of smoke and soot. Reference 20 provides a correlation between the emissive power of the fire, $E_{f}$, in W/m $/ \mathrm{m}^{2}$ and the diameter of the fire, $d$, in $\mathrm{ft}$ :

$$
E_{f}=E_{m} e^{-S d}+E_{s}\left(1-e^{-s d}\right)
$$

where $E_{m}$ is the emissive power of the luminous spots (equal to $140 \mathrm{~kW} / \mathrm{m}^{2}$ ), $E_{S}$ is the emissive power of the smoke (equal to $20 \mathrm{~kW} / \mathrm{m}^{2}$ ), and $S$ is an empirical coefficient (equal to 0.12 $\mathrm{m}^{-1}$ ). Equations 9-6 and 9-7 can be used to solve for $T$ in terms of $d$; the result is shown in Figure 9-6. This correlation was used in the DPTRA study if the object being heated is out of the fire.

\subsubsection{Fire Separation}

The fire separation is the closest distance between the outer perimeter of the fire and any surface of the trailer of the case vehicle. Distributions for the fire separation category and the separation distance were developed directly from the TIFA fire severity file. For cases involving multiple sources, only the most threatening fire ${ }^{17}$ was included in the data used to determine the distribution. The MHE was determined by linking the TIFA fire severity file and the main accident file constructed from TIFA. Table 9-2 shows the frequency of each separation category by MHE for fatal accidents. Figure 9-7 shows distributions of separation distance by MHE given that the separation category is standoff.

Table 9-2 Frequency of Separation Category by MHE (Fatal Accidents)

\begin{tabular}{|l|c|c|c|c|c|}
\hline \multirow{2}{*}{$\begin{array}{l}\text { Separation } \\
\text { Category }\end{array}$} & \multicolumn{5}{|c|}{ MHE } \\
\cline { 2 - 6 } & Truck/Tanker & Car & Objects & Train & Rollover/Fire/Immersion \\
\hline Engulfed & $39.81 \%$ & $50.47 \%$ & $71.74 \%$ & $41.67 \%$ & $65.88 \%$ \\
\hline Standoff & $60.19 \%$ & $49.53 \%$ & $28.26 \%$ & $58.33 \%$ & $34.12 \%$ \\
\hline
\end{tabular}

\subsubsection{Fire Size}

Because fire size was not well characterized in accident reports, an analytical simulation was developed to determine fire size distributions in Reference 1. The analytical model is a blend of pool and spill models. Certain parameters of the model were tuned using the accident data that were available. In this section, first the derivation of the analytical model and then a comparison of the analytical model with accident data is described.

\footnotetext{
${ }^{17}$ For the cases reviewed, the most threatening fire (as measured by duration, size, and proximity) was always the fire with the shortest standoff.
} 
For both pool and spill models, the fire can be represented as a right circular cylinder. Reference 21 documents several correlations that can be used to estimate the fire's height. For the DPTRA study, the height of the fire is taken to be three times its diameter, which is conservative.

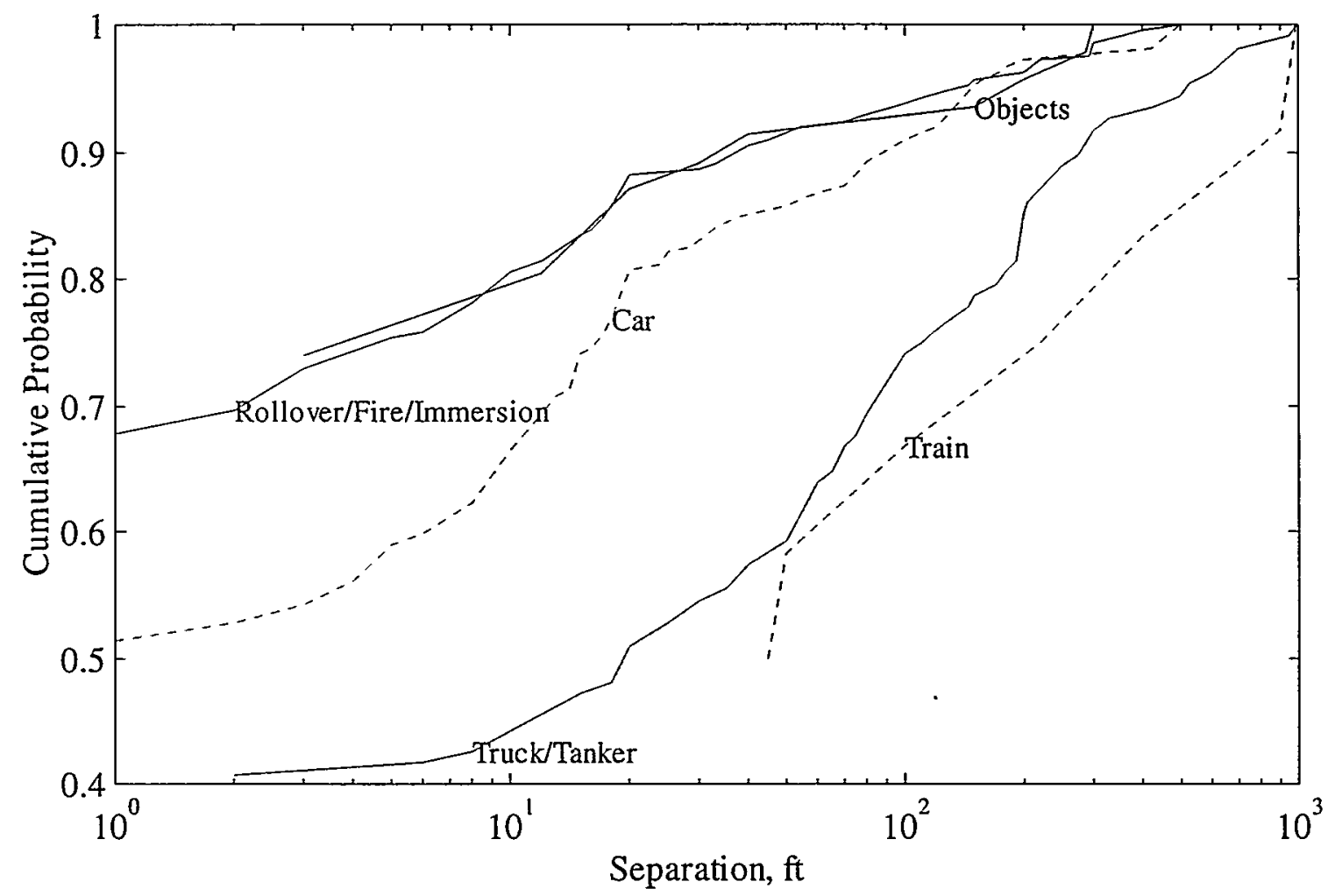

Figure 9-7 Distributions for Separation Distance by MHE

For pool fires, Reference 6 provides distributions for fire size that are dependent on the other vehicle involved in the accident. In the current study, the same form of distributions were used, but the parameters were altered to provide a better fit between the analytical model and the data.

Medium or heavy truck--fire size is uniformly distributed between 40 and $200 \mathrm{ft} 2$ :

$$
P_{1}\left(A_{p}\right)=\left(A_{p}-40\right) / 160, \quad 40 \leq A_{p} \leq 200
$$

Light truck or auto--fire size is uniformly distributed between 25 and $125 \mathrm{ft}^{2}$ :

$$
P_{2}\left(A_{p}\right)=\left(A_{p}-25\right) / 100, \quad 25 \leq A_{p} \leq 125
$$

Tanker--fire size is uniformly distributed between 50 and $500 \mathrm{ft}^{2}$ :

$$
P_{3}\left(A_{p}\right)=\left(A_{p}-50\right) / 450, \quad 50 \leq A_{p} \leq 500
$$

No other vehicles--fire size is uniformly distributed between 25 and $125 \mathrm{ft}^{2}$ : 


$$
P_{4}\left(A_{p}\right)=\left(A_{p}-25\right) / 100, \quad 25 \leq A_{p} \leq 125
$$

Train-fire size is Weibull distributed between 500 and $1000 \mathrm{ft}^{2}$ :

$$
P_{5}\left(A_{p}\right)=1-e^{-\left(\frac{A_{p}-500}{252}\right)^{4.6}}, \quad 500 \leq A_{p} \leq 1000
$$

In Eqns. 9-8 through 9-12, $A_{p}$ is the surface area of the pool fire in $\mathrm{ft}^{2}$ and $P_{i}\left(A_{p}\right)$ is the probability that the size of the fire is less than or equal to $A_{p}$ given that the other vehicle involved corresponds to that associated with index $i$.

The model for spill fires was developed by Marv Larsen based in part on Reference 22. The following description of spill fires borrows liberally from a quarterly progress report prepared by Larsen. ${ }^{18}$

As a liquid fuel first begins to drain out, the rate at which the fuel burns is less than the spill rate. As a result, the fire spreads along the surface onto which it is spilled. Given a constant spill rate on a flat surface and assuming a constant fuel surface recession (burn) rate, there results an equilibrium fire size which has just enough surface area to consume the supplied fuel. That is:

$$
Q=\pi R^{2} \dot{y},
$$

where $Q$ is the spill rate, $R$ is the diameter of the fire and $\dot{y}$ is the fuel surface recession rate. Rearranging Eqn. 9-13 to solve for $R$ results in:

$$
R=\sqrt{\frac{Q}{\pi \dot{y}}} .
$$

The spill is idealized as an opening at the bottom of a tank. If the tank is vented to the atmosphere, the Bernoulli Equation can be used to define the spill rate:

$$
Q=A_{l} v=A_{l} \sqrt{2 g h},
$$

where $A_{l}$ is the cross-sectional area of the opening through which the fuel spills (hereafter referred to as the leak area), $v$ is the velocity of the fuel through the opening, $g$ is the acceleration due to gravity, and $h$ is the height of the free surface of the fuel above the opening, which is clearly a function of time. The spill rate can also be related to the time rate of change in the volume of fuel in the tank:

\footnotetext{
${ }^{18}$ M. E. Larsen, "2nd-1/2 Quarterly Report (Contract 18-8453): Sandia Risk Assessment," South Dakota School of Mines and Technology, March 5, 1992.
} 


$$
Q=-\frac{d V}{d t}=-A_{t} \frac{d h}{d t}
$$

where $V$ is the volume of fuel in the tank, $t$ is time, and $A_{t}$ is the cross-sectional area of the tank (assumed to be constant). Equating the right hand sides of Eqns. 9-15 and 9-16, rearranging to permit integration by separation of variables, and integrating over the time necessary to completely drain the tank leads to:

$$
\int_{h_{0}}^{0} \frac{d h}{\sqrt{h}}=\sqrt{2 g} \frac{A_{l}}{A_{l}} \int_{0}^{t_{b}} d t
$$

where $h_{0}$ is the initial fuel height in the tank and $t_{b}$ is the time required to completely burn the fuel (which is equal to the time it takes to completely drain the tank). Completing the integration and solving for $t_{b}$ results in:

$$
t_{b}=\frac{A_{t}}{A_{l}} \sqrt{\frac{2 h_{0}}{g}}
$$

Eqn. 9-18 can be simplified further for tanks of particular shapes. For example,

$$
t_{b}=\frac{1.358 V^{5 / 6}}{A_{l} \sqrt{g}}
$$

for right circular cylinders of equal height and diameter (axis vertically aligned) and

$$
t_{b}=\frac{\sqrt{2} V^{5 / 6}}{A_{l} \sqrt{g}}
$$

for cubes. These examples suggest that the influence of tank geometry is relatively minor, and therefore Eqn. 9-20 is used to represent all tank geometries. The time-averaged spill rate, $\bar{Q}$, is given by:

$$
\bar{Q}=V / t_{b}
$$

Combining Eqns. 9-14, 9-20, and 9-21 leads to the following result for the time-averaged surface area associated with a spill fire:

$$
\overline{A_{s}}=\frac{V^{1 / 6} A_{l} \sqrt{g}}{\sqrt{2} \dot{y}} .
$$

$\overline{A_{s}}$ is used to approximate the surface area associated with a spill fire (i.e., the variation of the area with time not considered). The acceleration due to gravity, $g$, and the fuel surface recession rate, $\dot{y}$, are treated as constants in Eqn. 9-22. The fuel surface recession rate is taken 
to be $0.16 \mathrm{in} / \mathrm{min} .^{19}$ The volume of fuel, $V$, and the leak area, $A_{l}$, are treated as random variables with distributions that depend on the other vehicle involved. Given distributions for $V$ and $A_{l}$, distributions for $\overline{A_{s}}$ can be obtained using a Monte Carlo simulation. Note that the correlation between $\overline{A_{s}}$ and $V$ is relatively weak.

The distributions of $V$ are based on Reference 5. The distributions of $V$ when the other vehicle involved is a car, tanker, none and train are identical to those labeled car, tanker, single vehicle and train, respectively, in Reference 5. The distribution used for MHE equal to truck was modified from the corresponding distribution in Reference 5 to represent larger fuel tank capacities and the effect of combustible cargoes in the other truck. ${ }^{20}$ The resulting distributions for volume of fuel are:

Medium or heavy truck:

$$
P_{1}(V)=1-e^{-\left(\frac{V-50}{500}\right)^{0.8}}, \quad V \geq 50
$$

Light truck or auto:

$$
P_{2}(V)=1-e^{-\left(\frac{V}{166}\right)^{3.73}}, \quad V \geq 0
$$

Tanker:

$$
P_{3}(V)=1-e^{-\left(\frac{v}{5718}\right)^{2.73}}, \quad V \geq 0
$$

No other vehicles:

$$
P_{4}(V)=1-e^{-\left(\frac{V}{1.32}\right)^{3.68}}, \quad V \geq 0
$$

Train:

$$
P_{5}(V)=1-e^{-\left(\frac{V-1000}{1415}\right)^{1.06}}, \quad V \geq 1000
$$

\footnotetext{
${ }^{19}$ The fuel surface recession rate may vary depending on type of fuel, size of the fire, temperature of the fire, and ambient conditions, but the rate is typically between about 0.14 and $0.18 \mathrm{in} / \mathrm{min}$ for fires with diameters greater than $3-4 \mathrm{ft}$ (see Figure 1 of Reference 21 ). This variability is considered relatively small and is neglected in the models developed in this report.

${ }^{20} \mathrm{It}$ is assumed that the cargo in the vehicle of interest is not flammable, and thus the correction for the combustible cargo is only necessary in the case where the vehicle of interest is involved with another medium or heavy duty truck.
} 
In Eqns. 9-23 through 9-27, $V$ is the volume of fuel in gallons and $P_{i}(V)$ is the probability that the volume of fuel is less than or equal to $V$ given that the other vehicle involved corresponds to that associated with index $i$.

Neither accident data nor analytical models that can be used to directly determine $A_{l}$ exist. Therefore, Weibull distributions of $A_{l}$ that depend on the other type of vehicle ${ }^{21}$ are assumed; the parameters of these distributions were adjusted (within reasonable bounds) so that the resulting distribution of surface areas of fires was comparable to the limited accident data available on surface areas of fires. The procedure used to compare distributions is described shortly. The resulting distributions for leak area are:

Medium or heavy truck:

$$
P_{1}\left(A_{l}\right)=1-e^{-\left(\frac{A_{l}-0.1}{6}\right)^{4}}, \quad A_{l} \geq 0.1
$$

Light truck or auto:

$$
P_{2}\left(A_{l}\right)=1-e^{-\left(\frac{A_{l}-0.1}{5}\right)^{4}}, \quad A_{l} \geq 0.1
$$

Tanker:

$$
P_{3}\left(A_{l}\right)=1-e^{-\left(\frac{A_{l}-4}{10}\right)^{4}}, \quad A_{l} \geq 4
$$

No other vehicles:

$$
P_{4}\left(A_{l}\right)=1-e^{-\left(\frac{A_{t}-0.1}{5}\right)^{4}}, \quad A_{l} \geq 0.1
$$

Train:

$$
P_{5}\left(A_{l}\right)=1-e^{-\left(\frac{A_{l}-2}{8}\right)^{4}}, \quad A_{l} \geq 2
$$

In Eqns. 9-28 through 9-32, $A_{l}$ is the leak area in in ${ }^{2}$ and $P_{i}\left(A_{l}\right)$ is the probability that the leak area is less than or equal to $A_{l}$ given that the other vehicle involved corresponds to that associated with index $i$.

\footnotetext{
${ }^{21}$ Intuitively, the leak area depends on the collision severity, which in turns depends in part on the other vehicle involved. To maintain consistency with the distributions used to describe surface area for pool fires and the volume of fuel, $A_{l}$ was also assumed to depend only on the other vehicle involved, although in fact it may be correlated with other measures of collision severity (such as peak contact velocity) as well.
} 
Final distributions for surface area of fires are obtained from the combination of the pool and spill fire models as:

$$
P_{i}(A)=w_{p} P_{i}\left(A_{p}=A\right)+w_{s} P_{i}\left(\overline{A_{s}}=A\right) ;
$$

these distributions are dependent on the other vehicle involved as indicated by the index $i$. The pool and spill fire models are weighted by the factors $w_{p}$ and $w_{s}$, respectively. These weights were adjusted (within reasonable bounds) so that the resulting distribution of surface areas of fires was comparable to the limited accident data available on surface areas of fires. The values adopted in this study are $w_{p}=0.9$ and $w_{s}=0.1$.

The distributions for the size of the fire resulting from a Monte Carlo simulation based on the approach outlined above are shown in Figure 9-8. In this figure, the size of the fire is expressed in terms of its diameter, which is equal to $\sqrt{4 A / \pi}$. The distributions in Figure 9-8 are shown in terms of the MHE instead of the other vehicle involved. The relationship between the distributions in terms of the indicated MHE and the distribution in terms of the other vehicle involved are as follows:

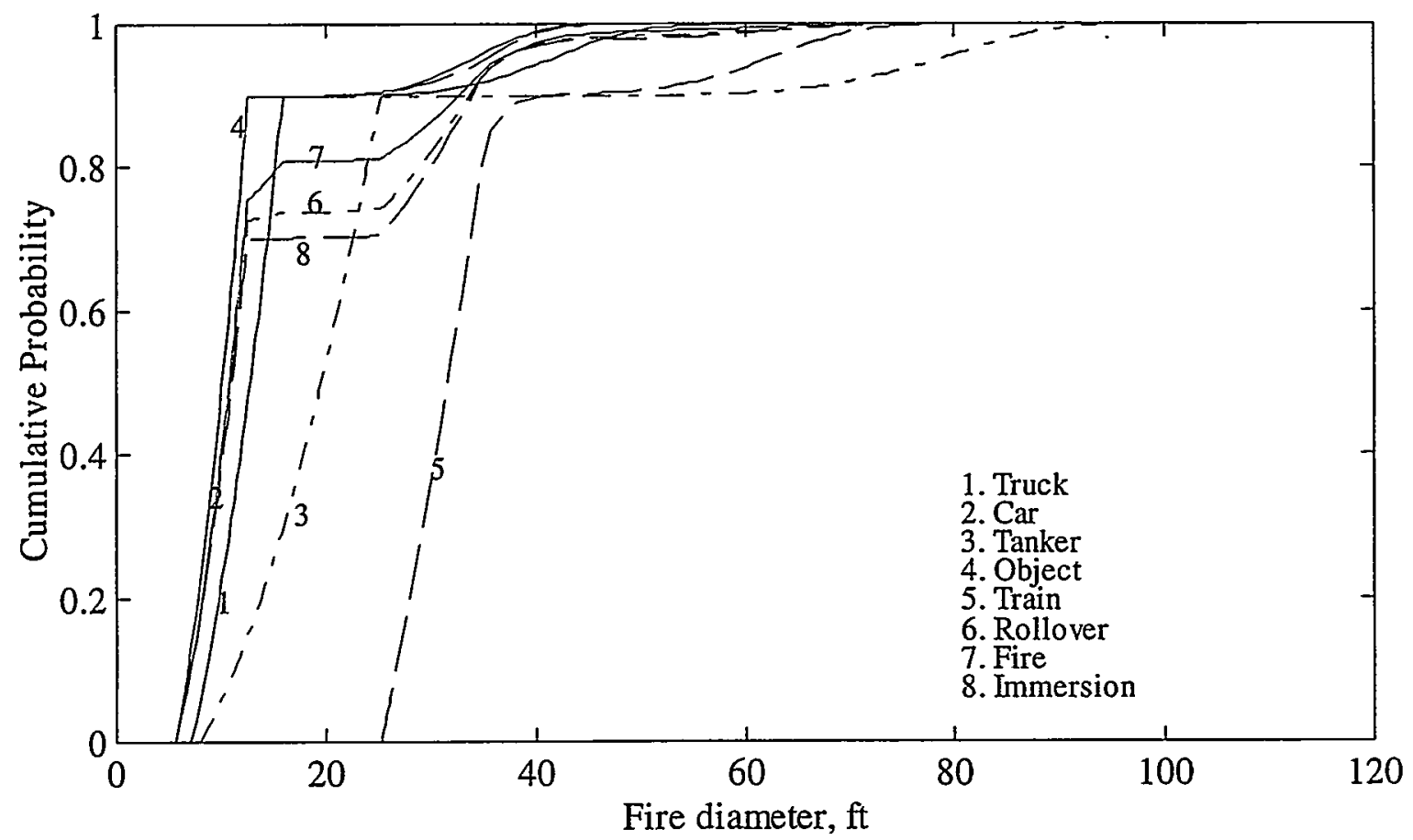

Figure 9-8 Fire Size Distributions

1. The distributions for fire size when the MHE is (1) collision with a medium or heavy truck, (2) collision with a light truck or auto, (3) involvement with a tanker, (4) collision with any object, and (5) collision with a train are identical to those when the other vehicle involved is a (1) truck, (2) car, (3) tanker, (4) none, and (5) train, respectively. 
2. The distributions for fire size when the MHE is rollover, fire or immersion were obtained by weighting the distributions for fire size when the MHE is truck, car, tanker, objects or train by the factors given in Table 9-3. The factors in Table 9-3 were obtained from consideration of the distribution of other vehicle involved by MHE given a fire occurrence, which is shown in Table 9-4. The weighting factors for truck, cars, and tankers were determined using the data in Table 9-4 directly. It was assumed that if the other vehicle involved was coded as none, that either an object or train was involved and that the likelihood of an object or train involvement is in proportion to the frequency with which these events are coded as the MHE given a fire. Thus, the weighting factor for objects was computed as the product of the frequency that no other vehicle is involved (as determined from Table 9-4) and the ratio of fatal involvements in which the MHE was coded as collision with an object and those in which the MHE was coded as either collision with an object or collision with a train (from Table 8-15).

Table 9-3 Weighting Factors Used to Construct Fire Size Distributions

\begin{tabular}{|l|c|c|c|c|c|}
\hline \multirow{2}{*}{ MHE } & \multicolumn{5}{|c|}{ Other Vehicle Involved } \\
\cline { 2 - 6 } & Truck & Cars & Tanker & Objects & Train \\
\hline Rollover & $2.63 \%$ & $15.79 \%$ & $0.00 \%$ & $63.58 \%$ & $18.00 \%$ \\
\hline Fire & $12.64 \%$ & $41.38 \%$ & $0.00 \%$ & $35.84 \%$ & $10.14 \%$ \\
\hline Immersion & $0.00 \%$ & $0.00 \%$ & $0.00 \%$ & $77.94 \%$ & $22.06 \%$ \\
\hline
\end{tabular}

Table 9-4 Fatal Involvements by Other Vehicle Involved and MHE Given a Fire Occurrence

\begin{tabular}{|l|c|c|c|c|}
\hline \multirow{2}{*}{ MHE } & \multicolumn{4}{|c|}{ Other Vehicle Involved } \\
\cline { 2 - 5 } & None & Truck & Car & Tanker \\
\hline Rollover & 31 & 1 & 6 & 0 \\
\hline Fire & 80 & 22 & 72 & 0 \\
\hline Immersion & 1 & 0 & 0 & 0 \\
\hline
\end{tabular}

The validity of these analytical simulations can be judged against the limited accident data available. Only the overall distributions for the surface area and duration of fires can be easily compared. Fire duration is considered the more important parameter, and so greater emphasis was placed on obtaining a good comparison between the analytical simulation and the accident data for fire duration.

Distributions for estimates of fire surface area as given in the TIFA supplemental data file are compared to a weighted distribution of fire surface areas based on the Monte Carlo simulation in Figure 9-9. The weighted distribution for the analytical model is obtained as:

$$
P(A)=\sum_{i} K_{i} P_{i}(A)
$$


where $K_{i}$ are weighting factors that apply to the probability that the other vehicle involved corresponds to that associated with the index $i$. In order to provide a valid comparison, the $K_{i}$ are based on the distribution of other vehicle involved for fatal accidents involving a fire. The $K_{i}$ are $.220, .506, .006, .209$ and .059 for $i=1-5$ (truck, car, tanker, none, train), respectively. Comparison of the results in Figure 9-9 suggests that the choice of distributions for the leak areas associated with spill fires and the weighting factors applied to the pool and spill fire models are reasonable. Comparison of the available accident data for fire duration with the analytical simulation provides even stronger support for this conclusion, as shown in the next section.

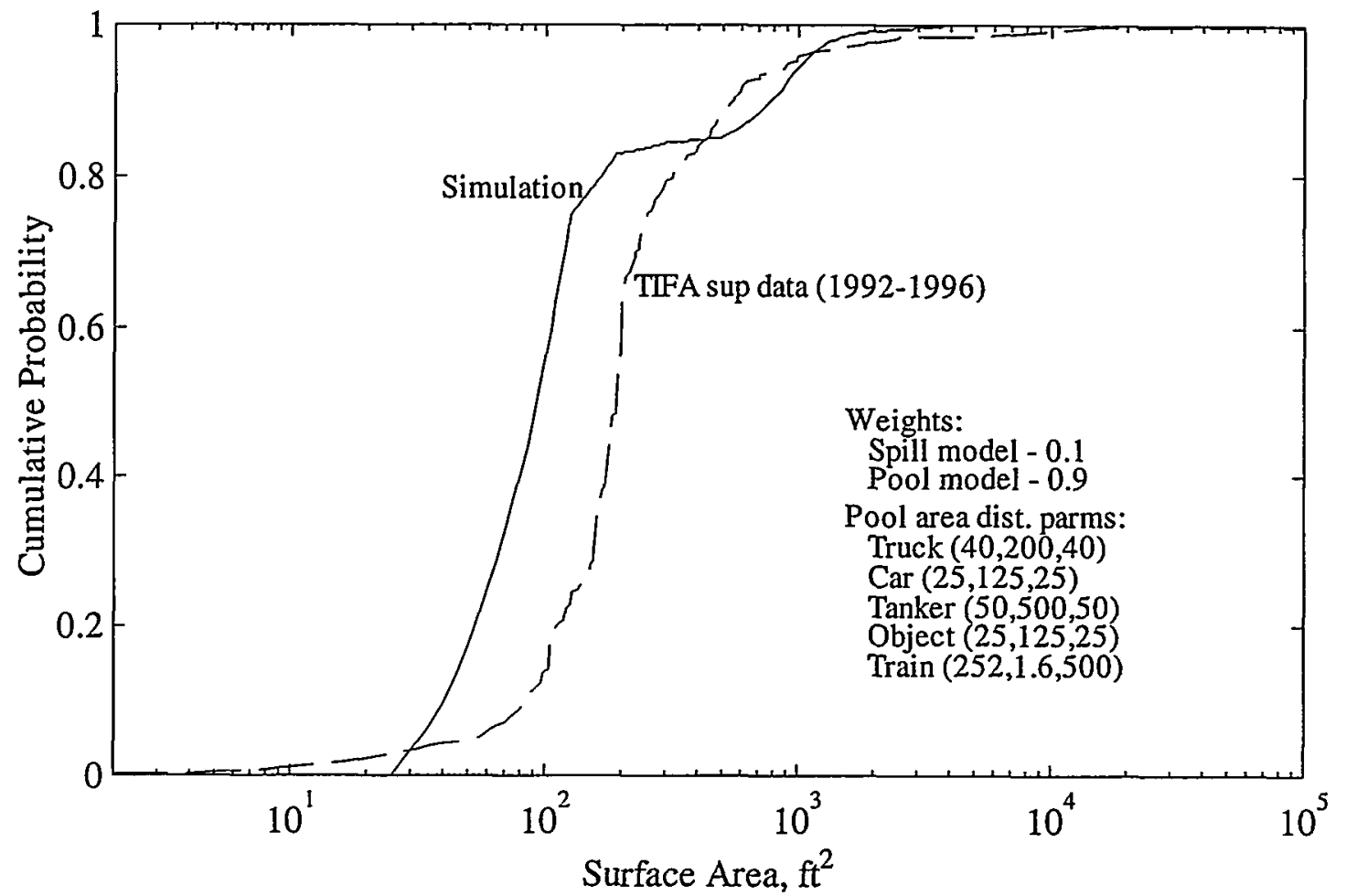

Figure 9-9 Comparison of Distributions for Fire Surface Area--Simulation vs. Accident Data

\subsubsection{Fire Duration}

The time necessary to completely burn all fuel, $t_{b}$, is:

$$
t_{b}=\frac{V}{A \dot{y}},
$$

where $V$ is the volume of fuel, $A$ is the surface area of the fire, and $\dot{y}$ is the fuel surface recession rate. The fuel surface recession rate is taken to be $0.16 \mathrm{in} / \mathrm{min}$, just as it is in Eqn 9-22. As described in the previous section, $V$ and $A$ are defined by distributions that depend on the other vehicle involved. Thus, distributions of $t_{b}$ that also depend on the other vehicle involved, $P_{i}\left(t_{b}\right)$, can be determined from a Monte Carlo simulation. Although $A$ is 
weakly dependent on $V$, this correlation was ignored in the Monte Carlo simulation used to develop distributions of $t_{b}$.

In this study, the time to completely burn all fuel, $t_{b}$, is used as an approximate measure of the fire duration. In previous studies, distributions of fire duration were developed based on the time to completely burn all fuel, but an adjustment was made to account for the effects of firefighting efforts [4-6]. The effects of fire-fighting were considered in this study. Models of fire-fighting based on Reference 6 were developed, but since the effect of including firefighting in the analytical model on the overall distribution for fire duration is not very significant, especially in comparison with the uncertainty in the accident data, the additional complexity associated with including the effects of fire-fighting in the analytical model was not warranted. Thus, the analytical simulation for fire duration in this study does not incorporate the effects of fire-fighting, which tends to give a conservative result.

Figure 9-10 through Figure 9-14 show distributions for fire duration based on a Monte Carlo simulation for $t_{b}$ for accidents in which the other vehicle involved is a truck, car, tanker, object, and tanker, respectively. Each figure includes a set of curves that represent distributions for specific values of fire diameter.

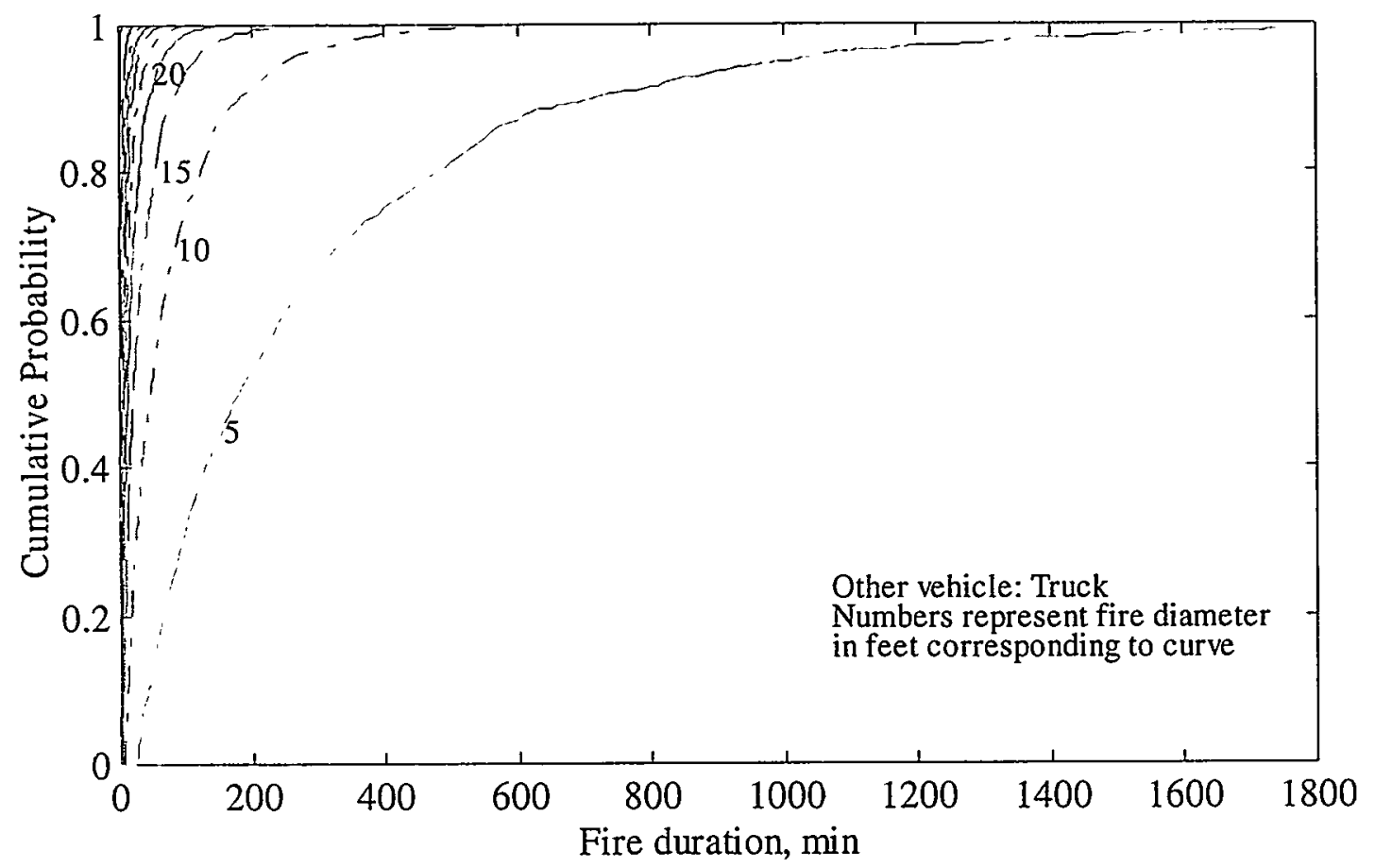

Figure 9-10 Fire Duration Distributions (Other Vehicle Involved is Truck) 


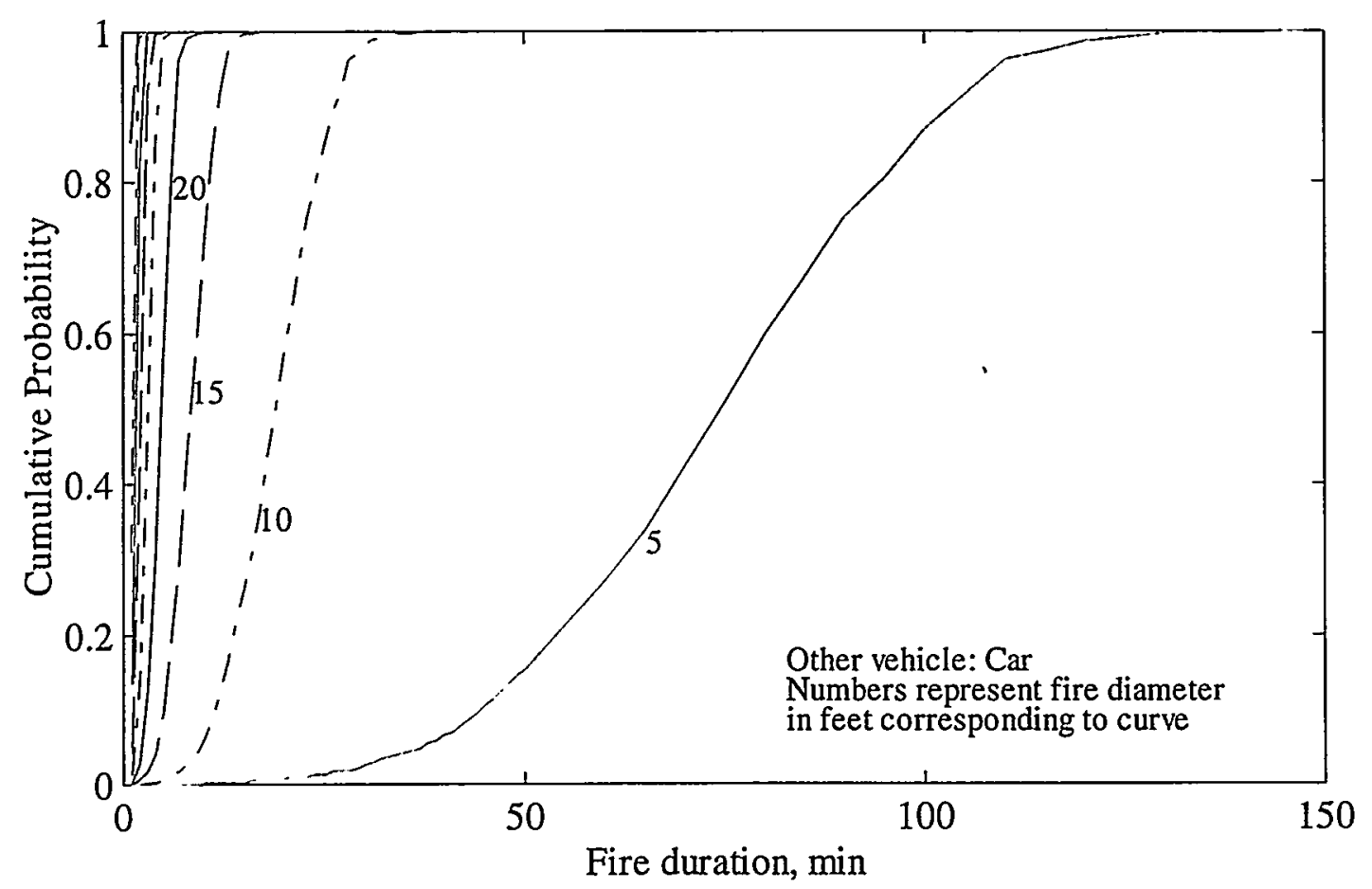

Figure 9-11 Fire Duration Distributions (Other Vehicle Involved is Car)

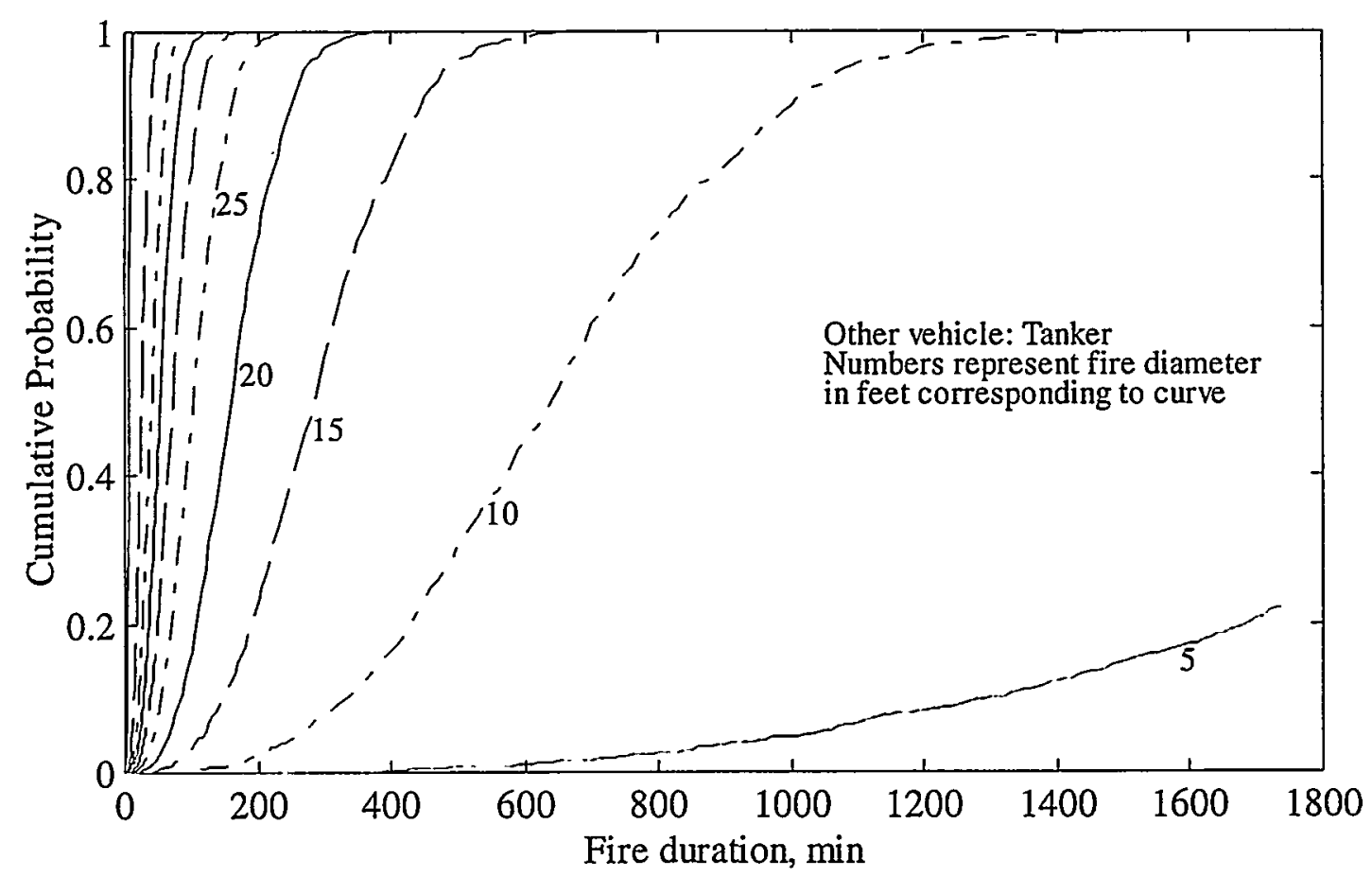

Figure 9-12 Fire Duration Distributions (Other Vehicle Involved is Tanker) 

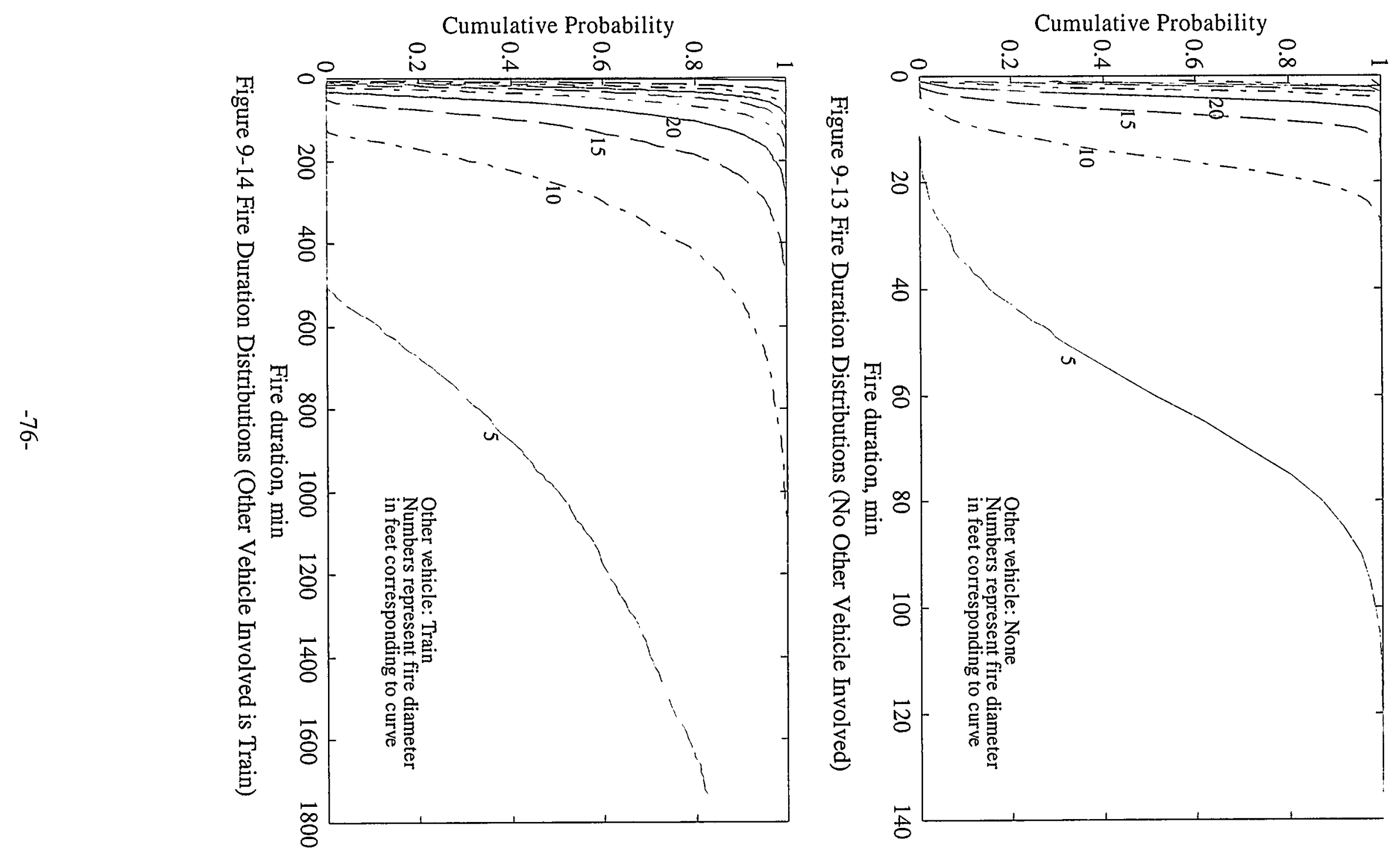
The validity of these analytical simulations can be judged against accident data. Only the overall distributions for the duration of fires can be easily compared. Distributions for estimates of fire duration as given in the TIFA supplemental data file are compared to a weighted distribution of fire durations based on the Monte Carlo simulation in Figure 9-15. The weighted distribution for the analytical model is obtained as:

$$
P(t)=\sum_{i} K_{i} P_{i}\left(t_{b}\right)
$$

where $K_{i}$ are weighting factors that apply to the probability that the other vehicle involved corresponds to that associated with the index $i$. In order to provide a valid comparison, the $K_{i}$ are based on the distribution of other vehicle involved for those accidents for which the duration of the fire is not unknown. The $K_{i}$ are the same as those used in the previous section, i.e., $0.33,0.31,0,0.30$ and 0.06 for $i=1-5$ (truck, car, tanker, none, train), respectively. Comparison of the results in Figure 9-15 in conjunction with those in Figure 9-9 suggests that the choice of distributions for the leak areas associated with spill fires and the weighting factors applied to the pool and spill fire models are appropriate and valid.

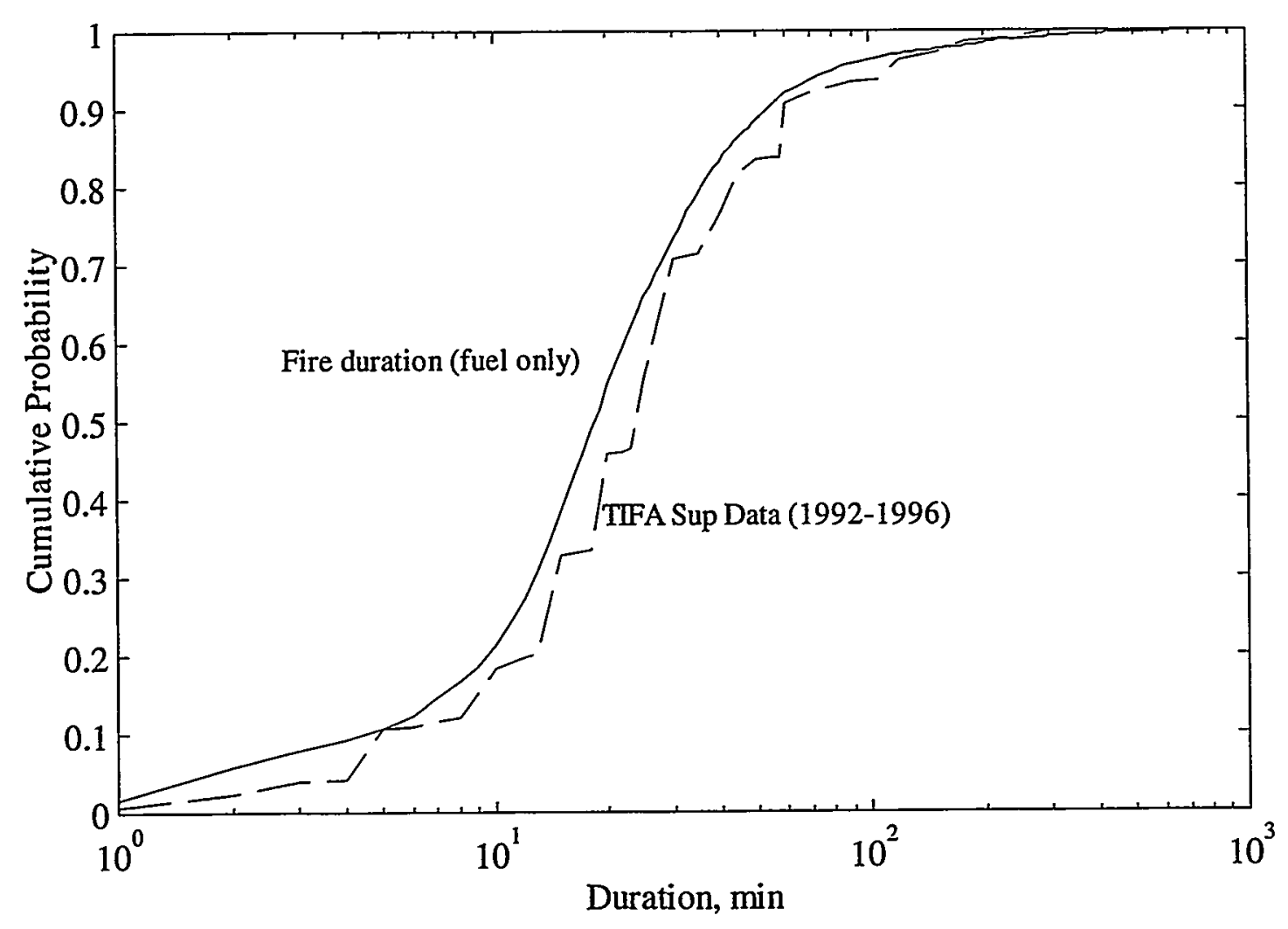

Figure 9-15 Comparison of Distributions for Fire Duration--Simulation vs. Accident Data 
--This page left almost blank intentionally-- 


\section{Application of Accident Data in Risk Assessment}

From the description and review of existing data sources in Chapter 5, it is clear that the TIFA file provides the best match with the risk assessment requirements. TIFA, since it includes the FARS accident level variables and extensive physical detail collected by telephone interviews, contains most of the variables and code levels necessary to support a detailed description of accident types. In addition, police reports were available and interviews were used to obtain additional information on accident severity for many TIFA cases, as described in Chapters 69.

The primary drawback to TIFA is that it is limited to fatal accidents. This limitation does not affect the utility of the TIFA file or the TIFA supplemental data in terms of describing the distribution of accident types and accident severities. As shown in Chapter 8, the distribution of accident types based on fatal accidents from the TIFA file is generally comparable to that based on tow-away accidents based on the GES file. The discussion in Chapter 9 demonstrated that, in general, fatal accidents pose a greater threat to hazardous cargoes than non-fatal accidents. However, a risk assessment based only on fatal accidents may not be comprehensive; i.e., it may be possible for some accidents that do not involve a fatality to pose a threat to hazardous cargoes. While fatal accidents typically involve collisions at high speeds, and so are of primary interest in evaluating threats to the integrity of the AT/SST, an analysis limited to fatal accidents may not encompass all the accidents of interest.

By contrast, any accident that could pose a threat to the containment of hazardous cargoes is almost certain to involve a tow-away of at least one vehicle or a fatality. However, the data on types and severities of accidents involving a tow-away or fatality (hereafter referred to as towaway accidents) is not as detailed or comprehensive as that for fatal accidents.

In DPTRA, these concerns were addressed by basing the overall accident rate on tow-away accidents and considering the fraction of tow-away accidents with severities comparable to fatals. The distribution of accident types and severities based on TIFA and TIFA supplemental data is appropriately used with the product of the overall tow-away accident rate and the fraction of tow-away accidents with severities comparable to fatals. This approach provides consideration of all accidents that could pose a threat to the containment of hazardous cargoes and a detailed characterization of accident types and severities.

The GES file provides information that can be used to estimate the percent of tow-away accidents that have severities comparable to fatal accidents. The estimates are approximate and subject to large uncertainties.

The number of heavy truck accident records in the GES file is somewhat limited but it does include accidents ranging from minor property damage to tow-away accidents to fatalities. The GES database includes fields that describe the maximum injury severity, the damage severity and whether or not a vehicle was towed. For tow-away accidents involving a tractor semi-trailer, the distribution of accidents by damage severity and injury severity based on GES 
data is shown in Table 10-1. Columns 2-5 list the percentages of all accidents that have a maximum injury severity as indicated by the corresponding row in column 1 that also have the indicated damage severity. By contrast, column 6 lists the percentage of all tow-away accidents that have the indicated maximum injury severity. Comparison of rows 3 and 5 for columns 2-5 of Table 10-1 indicates that fatal accidents are more severe than tow-away accidents in general. This observation is also supported by comparisons of the severities of non-fatal accidents based on police reported accidents from the state of Michigan and fatal accidents based on TIFA, as discussed in Reference 1. Unfortunately, the damage severity categories are somewhat ambiguous. In particular, the most severe category (disabling) may include anything from radiator damage to total demolition of the vehicle.

Table 10-1 Distribution of Tow-Away Accidents by Injury Severity and Damage Severity

\begin{tabular}{|c|c|c|c|c|c|}
\hline \multirow{2}{*}{$\begin{array}{c}\text { Maximum } \\
\text { Injury Severity }\end{array}$} & \multicolumn{4}{|c|}{ Damage Severity } & \multirow[b]{2}{*}{ Any } \\
\hline & None & Minor & Functional & Disabling & \\
\hline No Injury & $13.4 \%$ & $39.7 \%$ & $21.2 \%$ & $25.7 \%$ & $46.4 \%$ \\
\hline Injury & $7.7 \%$ & $39.3 \%$ & $25.5 \%$ & $27.5 \%$ & $49.0 \%$ \\
\hline Fatality & $1.5 \%$ & $17.3 \%$ & $24.5 \%$ & $56.6 \%$ & $2.8 \%$ \\
\hline Other & $14.6 \%$ & $54.1 \%$ & $21.8 \%$ & $9.5 \%$ & $1.9 \%$ \\
\hline Any & $10.3 \%$ & $39.1 \%$ & $23.4 \%$ & $27.2 \%$ & $100.0 \%$ \\
\hline
\end{tabular}

Two approaches were considered for estimating the percentage of tow-away accidents that have severities comparable to fatals. The first approach assumes that the severity of accidents that do not result in a fatality is substantially lower than the severity of fatal accidents and that non-fatal accidents do not pose a threat to the containment of hazardous cargoes. As can be seen from Table $10-1,2.8 \%$ of tow-away accidents also involve a fatality. The second approach is based on excluding an appropriate percentage of tow-aways such that the distribution of damage severity for fatals is replicated. This can be accomplished by screening out $92.8 \%$ of tow-aways involving no damage, $78.68 \%$ of those with minor damage, and $49.64 \%$ of those with functional damage (retaining all those that are disabling). The net result is that by retaining $48.1 \%$ of tow-aways, the distribution of damage severity for fatals can be matched. The damage levels used in the GES file are somewhat ambiguous and disabling accidents for tow-away accidents may or may not be nearly as severe, in general, as those for fatal accidents. Thus, the two approaches described represent a lower and upper bound estimate, respectively, for the fraction of tow-aways with severities comparable to fatals. 


\section{Summary}

This report provides distributions of the types and severities of accidents involving tractor semi-trailers that can be used in risk assessments for transportation of hazardous cargoes. The primary data are based on traffic accidents involving at least one fatality. The information presented in this report was specifically developed to support a probabilistic assessment of the risk of transporting certain radioactive materials in the AT/SST for the DOE, but application to other carriers and other types of hazardous cargoes may be possible if appropriate adjustments are made in the data.

Distributions of accident types as defined by the types of vehicles and/or other objects involved, collision occurrence, angle of impact, location of principal impact, rollover occurrence and fire occurrence are described in Chapter 8. Distributions of peak contact velocity, skid distance, effective fire temperature, fire size, fire separation, and fire duration were developed to define the severity of the accident, as discussed in Chapter 9. The report also includes a discussion of the application of this data in risk assessments for transportation of hazardous cargoes in Chapter 10. 
--This page left almost blank intentionally--

$-82-$ 


\section{References}

[1] D. B. Clauss, et al., "A Statistical Description of the Types and Severities of Accidents Involving Tractor Semi-Trailers," SAND93-2580, Sandia National Laboratories, Albuquerque, New Mexico, June 1994.

[2] J. S. Phillips, D. B. Clauss and D. F. Blower, "Determination of Influence Factors and Accident Rates for the Armored Tractor/Safe Secure Trailer," SAND93-0111, Sandia National Laboratories, Albuquerque, New Mexico, June 1993.

[3] J. M. Clauss and E. K. Lemen, "Defense Programs Transportation Risk Assessment (DPTRA '99) Input Data: Over-the-Road Shipment Projections, Routes, Accident Rate, and Influence Factors," draft report, Sandia National Laboratories, Albuquerque, New Mexico, to be published.

[4] L. E. Fischer et al., "Shipping Container Response to Severe Highway and Railway Accident Conditions," NUREG/CR-4829, UCID-20733, Lawrence Livermore National Laboratories, February 1987.

[5] A. W. Dennis, J. T. Foley, W. F. Hartman and D. W. Larson, "Severities of Transportation Accidents Involving Large Packages," SAND77-0001, Sandia National Laboratories, Albuquerque, New Mexico, May 1978.

[6] R. K. Clarke, J. T. Foley, W. F. Hartman and D. W. Larson, "Severities of Transportation Accidents," SLA-74-0001, Sandia Laboratories, Albuquerque, New Mexico, July 1976.

[7] National Center for Statistics and Analysis, "National Accident Sampling System (NASS), Crashworthiness Data Subsystem, Analytical User's Manual,” 1989.

[8] "Office of Motor Carriers, 1987, Codebook," Transportation Data Center, University of Michigan Transportation Research Institute, Number 89-19, September, 1989.

[9] K. P. Sullivan and D. L. Massie, "Trucks Involved In Fatal Accidents, 1991 Factbook," UMTRI-94-35, University of Michigan Transportation Research Institute, October 1994.

[10] "Fatal Accident Reporting System, 1996, Codebook, Version ARF," Transportation Data Center, University of Michigan Transportation Research Institute, Number 97-3, August 1997.

[11] "Traffic Safety Facts 1996, A Compilation of Motor Vehicle Crash Data from the Fatality Analysis Reporting System and the General Estimates System," DOT-HS-808649, U.S. Department of Transportation, National Highway Traffic Safety Administration, December 1997. 
[12] "Michigan Codebook, 1990," Transportation Data Center, University of Michigan Transportation Research Institute, Number 91-6, June 1991.

[13] L. B. Fricke, "Traffic Accident Reconstruction," Northwestern University Traffic Institute, 1990.

[14] J. F. Marquardt, "Vehicle and Occupant Factors that Determine Occupant Injury," Society of Automotive Engineers, Automotive Engineering Congress, Detroit, MI, February 25-March 1, 1974.

[15] J. D. McClure et al., "Relative Response of Type B Packagings to Regulatory and Other Impact Test Environments," Proceedings of the $6^{\text {th }}$ International Conference on Packaging and Transportation of Radioactive Materials, pp 1247-1253, Berlin, Germany, November 10-14, 1980.

[16] D. Blower, L. Pettis, "Trucks Involved In Fatal Accidents, Codebook 1996" UMTRI-9814, University of Michigan Transportation Research Institute, May 1998.

[17] "NASS General Estimate System 1989 Codebook - Version 120390," Transportation Data Center, University of Michigan Transportation Research Institute, Number 91-2, February 1991.

[18] M. E. Larsen, "MELTER: A Model of the Thermal Response of Cargoes Transported in the Safe-Secure Trailer Subject to Fire Environments for Risk Assessment Applications," SAND93-0737, Sandia National Laboratories, September, 1993, DRAFT.

[19] D. R. Stephens, "Fire Resistant Pits: Reducing the Probability of Accidental Plutonium Dispersal from Fuel Fires," UCRL-ID-110556, Lawrence Livermore National Laboratory, March 1992.

[20] National Fire Protection Association, "The SFPE Handook for Fire Protection Engineering," 1st edition, P. J. Dinenno, ed., SFPE 8-88.

[21] K. S. Mudan, "Thermal Radiation Hazards from Hydrocarbon Pool Fires," Prog. Energy Combust. Sci., 10, pp. 59-80, 1984.

[22] J. A. Mansfield and L. J. Linley, "Measurement and Statistical Analysis of Flame Temperatures from Large Fuel Spill Fires," Naval Weapons Center Technical Publication 7061, Naval Weapons Center, China Lake, CA, January 1991. 


\section{Acronyms}

AT-armored tractor

DOE-U.S. Department of Energy

DOT-U.S. Department of Transportation

DPTRA-Defense Programs Transportation Risk Assessment

FARS-Fatal Accident Reporting System

FHWA-Federal Highway Administration

GCW-gross combination weight

GES-General Estimates System

GR-guardrail

GVWR-gross vehicle weight rating

LBE-lower bound estimate

MHE-most harmful event

NASS-National Accident Sample Survey

NHTSA-National Highway Traffic Safety Administration

OMC-Office of Motor Carriers

PSU-Probability Sampling Unit

SGT - safeguards trailer

SST-safe secure trailer

TIFA-Trucks Involved in Fatal Accidents

UBE-upper bound estimate

UMTRI-University of Michigan Transportation Research Institute

VIN-vehicle identification number 
--This page left almost blank intentionally-- 


\section{Nomenclature}

A surface area of a fire

$A_{t} \quad$ cross-sectional area of the fuel tank

$A_{l} \quad$ leak area (cross-sectional area of opening)

$A_{p} \quad$ surface area of a pool fire

$\overline{A_{s}} \quad$ time-averaged surface area of a spill fire

$d \quad$ diameter of the fire

$D \quad$ skid distance after a rollover

E collision energy absorbed by the AT/SST

$E_{a} \quad$ total energy absorbed in a collision

$E_{f} \quad$ emissive power of the fire

$E_{m} \quad$ emissive power of the luminous spots

$E_{S} \quad$ emissive power of the smoke

$g \quad$ acceleration due to gravity

$h$ height of the free surface of the fuel above the opening

$h_{0} \quad$ initial fuel height

$K_{i} \quad$ distribution weighting factors

$M \quad$ effective mass of the case vehicle

$m \quad$ effective mass of the other vehicle or object involved

$P \quad$ cumulative probability function

$P C V$ peak contact velocity of the case vehicle

pcv peak contact velocity of the other vehicle or object involved

$Q \quad$ fuel spill rate

$\bar{Q} \quad$ time-averaged spill rate

$R \quad$ diameter of a fire

$S \quad$ empirical coefficient used in evaluation of emissive power of the fire

$t \quad$ time

$t_{b} \quad$ time required to completely burn the fuel

$t_{d} \quad$ collision duration

$T \quad$ effective fire temperature

$V \quad$ volume of fuel

$V_{r} \quad$ relative velocity or closing velocity 


$\begin{array}{ll}v & \text { velocity of fuel through an opening } \\ w_{p} & \text { weighting factor for pool fire model } \\ w_{s} & \text { weighting factor for spill fire model } \\ \dot{y} & \text { fuel surface recession rate } \\ \sigma & \text { Stefan-Boltzman constant }\end{array}$




\section{Distribution}

2 U.S. Department of Energy

Attn.: D. K. Larsen, DP-22 (1)

H. Filacchione, DP-21 (1)

19901 Germantown Road

Germantown, MD 20874-1290

$7 \quad$ U.S. Department of Energy

Albuquerque Operations Office

Attn.: A. D. Luck, TSD/AB (1)

S. D. Northrop, WPD (1)

L. M. Pace, SASD (1)

N. A. Sandoval, SASD (1)

S. A. Thompson, WSD (2)

R. D. Williams, DOE/TSD/SMS (1)

P.O. Box 5400

Albuquerque, NM 87185-5400

1 Los Alamos National Laboratory

Attn.: E. I. Whitted, TSA-5, MS F602 (1)

P.O. Box 1663

Mail Station 5000

Los Alamos, NM 87545

2 University of California

Lawrence Livermore National Laboratory

Attn.: L. P. Altbaum, L-125 (1)

K. A. Peterman, L-125 (1)

P.O. Box 808

Livermore, CA 94550

1 Hartman Consulting

Attn: W. F. Hartman (1)

37241 S. Canyon View Road

Tucson, AZ 85739

10 UMTRI - University of Michigan

Transportation Research Institute

Attn: D. F. Blower (5)

R. A. Pichler (5)

2901 Baxter Road

Ann Arbor, MI 48109-2150 


$\begin{array}{rrl}1 & \text { MS0492 } & \text { D. R. Olson, 12332 } \\ 1 & \text { MS0718 } & \text { C. D. Massey, 6331 } \\ 1 & \text { MS0718 } & \text { M. B. Parks, 6331 } \\ 5 & \text { MS0718 } & \text { L. Shyr, 6331 } \\ 5 & \text { MS0718 } & \text { J. M. Clauss, 6331 } \\ 10 & \text { MS0767 } & \text { D. B. Clauss, 6314 } \\ 1 & \text { MS9018 } & \text { Central Technical Files, 8940-2 } \\ 2 & \text { MS0899 } & \text { Technical Library, 4916 } \\ 1 & \text { MS0612 } & \text { Review \& Approval Desk, 4912 } \\ & & \text { (For DOE/OST) }\end{array}$

$-90-$ 